\title{
RIESZ TRANSFORMS AND MULTIPLIERS FOR THE BESSEL-GRUSHIN OPERATOR
}

\author{
V. ALMEIDA, J.J. BETANCOR, A.J. CASTRO, AND K. SADARANGANI
}

Abstract. We establish that the spectral multiplier $\mathfrak{M}\left(G_{\alpha}\right)$ associated to the differential operator

$$
G_{\alpha}=-\Delta_{x}+\sum_{j=1}^{m} \frac{\alpha_{j}^{2}-1 / 4}{x_{j}^{2}}-|x|^{2} \Delta_{y} \text { on }(0, \infty)^{m} \times \mathbb{R}^{n},
$$

which we denominate Bessel-Grushin operator, is of weak type $(1,1)$ provided that $\mathfrak{M}$ is in a suitable local Sobolev space. In order to do this we prove a suitable weighted Plancherel estimate. Also, we study $L^{p}$-boundedness properties of Riesz transforms associated to $G_{\alpha}$, in the case $n=1$.

\section{INTRODUCTION}

If $m, n \in \mathbb{N}$ the Grushin operator on $\mathbb{R}^{m} \times \mathbb{R}^{n}$ is defined by

$$
G=-\sum_{j=1}^{m} \frac{\partial^{2}}{\partial x_{j}^{2}}-\left(\sum_{j=1}^{m} x_{j}^{2}\right)\left(\sum_{j=1}^{n} \frac{\partial^{2}}{\partial y_{j}^{2}}\right) .
$$

In this paper we consider the operators we call Bessel-Grushin operators which appear when Bessel operators replace the first Laplacian operator in $G$. If $\beta>-1 / 2$ the Bessel operator is defined by

$$
B_{\beta}=\frac{d^{2}}{d x^{2}}-\frac{\beta^{2}-1 / 4}{x^{2}}, \quad x \in(0, \infty) .
$$

Let $m, n \in \mathbb{N}$ and $\alpha=\left(\alpha_{1}, \ldots, \alpha_{m}\right) \in(-1 / 2, \infty)^{m}$. We introduce the Bessel-Grushin operator $G_{\alpha}$ as follows

$$
G_{\alpha}=-\sum_{j=1}^{m} B_{\alpha_{j}}-\left(\sum_{j=1}^{m} x_{j}^{2}\right)\left(\sum_{j=1}^{n} \frac{\partial^{2}}{\partial y_{j}^{2}}\right), \text { on }(0, \infty)^{m} \times \mathbb{R}^{n} .
$$

Our objective in this paper is to study $L^{p}$-boundedness properties of spectral multipliers and Riesz transforms associated with $G_{\alpha}$, being $n=1$ in the case of Riesz transforms. We are motivated by the recent papers of Chen and Sikora [5], Jotsaroop, Sanjay and Thangavelu [16], Martini and Muller [19] and Martini and Sikora [20, about Grushin operators.

We consider the Laguerre operator

$$
L_{\beta}=-\frac{d^{2}}{d x}+\frac{\beta^{2}-1 / 4}{x^{2}}+x^{2}, \text { on }(0, \infty) .
$$

where $\beta>-1 / 2$. We have that, for every $k \in \mathbb{N}$,

$$
L_{\beta} \varphi_{k}^{\beta}=2(2 k+\beta+1) \varphi_{k}^{\beta},
$$

where

$$
\varphi_{k}^{\beta}(x)=\left(\frac{2 \Gamma(k+1)}{\Gamma(k+\beta+1)}\right)^{1 / 2} e^{-x^{2} / 2} x^{\beta+1 / 2} l_{k}^{\beta}\left(x^{2}\right), \quad x \in(0, \infty),
$$

and $l_{k}^{\beta}$ represents the $k$-th Laguerre polynomial of order $\beta$ ([31, p. 100-102]). The family $\left\{\varphi_{k}^{\beta}\right\}_{k \in \mathbb{N}}$ is an orthonormal basis in $L^{2}(0, \infty)$.

Date: Thursday $18^{\text {th }}$ October, 2018.

2010 Mathematics Subject Classification. 42C, 42C10, 43A90.

Key words and phrases. Bessel operators, Grushin operators, spectral multipliers, Riesz transforms, Rboundedness.

The second and the third authors are partially supported by MTM2010/17974. The third author is also supported by a FPU grant from the Government of Spain. 
Let $\alpha=\left(\alpha_{1}, \ldots, \alpha_{m}\right) \in(-1 / 2, \infty)^{m}$. We define the Laguerre operator $\mathfrak{L}_{\alpha}$ on $(0, \infty)^{m}$ by

$$
\mathfrak{L}_{\alpha}=\sum_{j=1}^{m} L_{\alpha_{j}} .
$$

If for $k=\left(k_{1}, \ldots, k_{m}\right) \in \mathbb{N}^{m}$,

$$
\Phi_{k}^{\alpha}(x)=\prod_{j=1}^{m} \varphi_{k_{j}}^{\alpha_{j}}\left(x_{j}\right), \quad x=\left(x_{1}, \ldots, x_{m}\right) \in(0, \infty)^{m},
$$

we have that

$$
\mathfrak{L}_{\alpha} \Phi_{k}^{\alpha}(x)=2(2 \mathfrak{s}(k)+\mathfrak{s}(\alpha)+m) \Phi_{k}^{\alpha}(x) .
$$

where $\mathfrak{s}(k)=k_{1}+\ldots+k_{m}$ and $\mathfrak{s}(\alpha)=\alpha_{1}+\ldots+\alpha_{m}$.

The system $\left\{\Phi_{k}^{\alpha}(x)\right\}_{k \in \mathbb{N}^{m}}$ is an orthonormal basis in $L^{2}\left((0, \infty)^{m}\right)$.

We denote by $\mathcal{F}_{2}(f)$ the Fourier transform of $f \in L^{2}\left((0, \infty)^{m} \times \mathbb{R}^{n}\right)$ with respect to the second $\mathbb{R}^{n}$-variable, that is,

$$
\mathcal{F}_{2}(f)(x, y)=\frac{1}{(2 \pi)^{n / 2}} \int_{\mathbb{R}^{n}} e^{-i y \cdot z} f(x, z) d z, \quad(x, y) \in(0, \infty)^{m} \times \mathbb{R}^{n} .
$$

The Bessel-Grushin operator $\widetilde{G}_{\alpha}$ is defined by

$$
\widetilde{G}_{\alpha}(f)=\mathcal{F}_{2}^{-1}\left[\sum_{k \in \mathbb{N}^{m}} 2(2 \mathfrak{s}(k)+\mathfrak{s}(\alpha)+m)|u| c_{k}^{\alpha}\left(\mathcal{F}_{2}(f) ; u\right) \Phi_{k}^{\alpha}(x ;|u|)\right], \quad f \in D\left(\widetilde{G}_{\alpha}\right),
$$

where $\mathcal{F}_{2}^{-1}$ represents the inverse map of $\mathcal{F}_{2}$ and

$D\left(\widetilde{G}_{\alpha}\right)=\left\{f \in L^{2}\left((0, \infty)^{m} \times \mathbb{R}^{n}\right): \sum_{k \in \mathbb{N}^{m}}^{\infty} 2(2 \mathfrak{s}(k)+\mathfrak{s}(\alpha)+m)|u| c_{k}^{\alpha}\left(\mathcal{F}_{2}(f) ; u\right) \Phi_{k}^{\alpha}(x ;|u|) \in L^{2}\left((0, \infty)^{m} \times \mathbb{R}^{n}\right)\right\}$.

Here, for every $k \in \mathbb{N}^{m}$ and $g \in L^{2}\left((0, \infty)^{m} \times \mathbb{R}^{n}\right)$,

$$
c_{k}^{\alpha}(g ; u)=\int_{(0, \infty)^{m}} \Phi_{k}^{\alpha}(x ;|u|) g(x, u) d x, \quad u \in \mathbb{R}^{n},
$$

where

$$
\Phi_{k}^{\alpha}(x ; a)=a^{\frac{m}{4}} \Phi_{k}^{\alpha}(\sqrt{a} x), \quad x \in(0, \infty)^{m} \text { and } a>0 .
$$

Note that, for every $a>0,\left\{\Phi_{k}^{\alpha}(\cdot ; a)\right\}_{k \in \mathbb{N}^{m}}$ is an orthonormal basis in $L^{2}\left((0, \infty)^{m}\right)$. Moreover, denoting by $\mathfrak{L}_{\alpha}(a)$ the operator

$$
\mathfrak{L}_{\alpha}(a)=\sum_{j=1}^{m}\left(-B_{\alpha_{j}}+a^{2} x_{j}^{2}\right), \quad a>0
$$

we have that

$$
\mathfrak{L}_{\alpha}(a) \Phi_{k}^{\alpha}(\cdot, a)=2(2 \mathfrak{s}(k)+\mathfrak{s}(\alpha)+m) a \Phi_{k}^{\alpha}(\cdot ; a), \quad k \in \mathbb{N}^{m} \text { and } a>0 .
$$

If $f \in C_{c}^{\infty}\left((0, \infty)^{m} \times \mathbb{R}^{n}\right)$, the space of smooth functions with compact support in $(0, \infty)^{m} \times \mathbb{R}^{n}$, it is clear that $\widetilde{G}_{\alpha} f=G_{\alpha} f$. In the sequel we write also $G_{\alpha}$ to refer us to the operator $\widetilde{G}_{\alpha}$.

Suppose that $\mathfrak{M}$ is a bounded Borel function on $(0, \infty)$. We define the spectral multiplier $\mathfrak{M}\left(G_{\alpha}\right)$ associated with $\mathfrak{M}$ by

$\mathfrak{M}\left(G_{\alpha}\right) f=\mathcal{F}_{2}^{-1}\left[\sum_{k \in \mathbb{N}^{m}}^{\infty} \mathfrak{M}(2(2 \mathfrak{s}(k)+\mathfrak{s}(\alpha)+m)|u|) c_{k}^{\alpha}\left(\mathcal{F}_{2}(f) ; u\right) \Phi_{k}^{\alpha}(x ;|u|)\right], \quad f \in L^{2}\left((0, \infty)^{m} \times \mathbb{R}^{n}\right)$.

Since $\mathfrak{M}$ is bounded the operator $\mathfrak{M}\left(G_{\alpha}\right)$ is bounded from $L^{2}\left((0, \infty)^{m} \times \mathbb{R}^{n}\right)$ into itself. As usual, the question is to give conditions on the function $\mathfrak{M}$ such that the operator $\mathfrak{M}\left(G_{\alpha}\right)$ can be extended from $L^{2}\left((0, \infty)^{m} \times \mathbb{R}^{n}\right) \cap L^{p}\left((0, \infty)^{m} \times \mathbb{R}^{n}\right)$ to $L^{p}\left((0, \infty)^{m} \times \mathbb{R}^{n}\right)$ as a bounded operator from $L^{p}\left((0, \infty)^{m} \times \mathbb{R}^{n}\right)$ into itself, when $p \neq 2$.

In the classical Hörmander multiplier, local Sobolev norms are considered to describe smoothness of $\mathfrak{M}$ in order to get $L^{p}$-boundedness of the multiplier operator. These arguments have been used by Christ [6], Duong, Ouhabaz and Sikora [8], Duong, Sikora and Yen [9], Hebisch [15], Hulanicki and Stein [12, cf.], in different settings. 
If $1 \leq q \leq \infty$ and $s>0$, we denote by $W_{q}^{s}(\mathbb{R})$ the $L^{q}$-Sobolev space of order $s$. We choose $\eta \in C_{c}^{\infty}(0, \infty)$ not identically zero. The "local" $W_{q}^{s}$ norm $\|\cdot\|_{M W_{q}^{s}}$ is defined by

$$
\|\mathfrak{M}\|_{M W_{q}^{s}}=\sup _{t>0}\left\|\eta \delta_{t} \mathfrak{M}\right\|_{W_{q}^{s}}
$$

where $\delta_{t} \mathfrak{M}(s)=\mathfrak{M}(t s), t, s \in(0, \infty)$. When we consider different functions $\eta$ we get equivalent local Sobolev norms.

We now establish our result about spectral multipliers for Bessel-Grushin operators.

Theorem 1.1. Let $\alpha \in[1 / 2, \infty)^{m}$, and $D=\max \{m+n, 2 n\}$. Suppose that $\mathfrak{M}$ is a bounded Borel measurable function on $(0, \infty)$ such that $\|\mathfrak{M}\|_{M W_{2}^{s}}<\infty$ for $s>D / 2$. Then, the spectral multiplier $\mathfrak{M}\left(G_{\alpha}\right)$ can be extended from $L^{1}\left((0, \infty)^{m} \times \mathbb{R}^{n}\right) \cap L^{2}\left((0, \infty)^{m} \times \mathbb{R}^{n}\right)$ to $L^{1}\left((0, \infty)^{m} \times \mathbb{R}^{n}\right)$ as a bounded operator from $L^{1}\left((0, \infty)^{m} \times \mathbb{R}^{n}\right)$ into $L^{1, \infty}\left((0, \infty)^{m} \times \mathbb{R}^{n}\right)$.

Note that when $\mathfrak{M}$ is a bounded measurable function on $(0, \infty), \mathfrak{M}\left(G_{\alpha}\right)$ is bounded from $L^{2}\left((0, \infty)^{m} \times \mathbb{R}^{n}\right)$ into itself, and then classical interpolation theorems, duality and Theorem 1.1 imply that the operator $\mathfrak{M}\left(G_{\alpha}\right)$ can be extended from $L^{p}\left((0, \infty)^{m} \times \mathbb{R}^{n}\right) \cap L^{2}\left((0, \infty)^{m} \times \mathbb{R}^{n}\right)$ to $L^{p}\left((0, \infty)^{m} \times \mathbb{R}^{n}\right)$ as a bounded operator from $L^{p}\left((0, \infty)^{m} \times \mathbb{R}^{n}\right)$ into it self, for every $1<p<\infty$, provided that $\mathfrak{M}$ satisfies the conditions in Theorem 1.1 .

According to [19, Theorem 1] we conjeture that when $m<n$ the result in Theorem 1.1 is also true for $s>(m+n) / 2$. Moreover, we also conjeture that the order $(m+n) / 2$ of differentiability in Theorem 1.1 cannot be decreased. In order to show this the idea is to work with the imaginary power $G_{\alpha}^{i t}, t \in \mathbb{R}$, of the Bessel-Grushin operator and to adapt the arguments in [29] (see [5, Section $5]$ and [20, Section 5]). We will study these questions in a foregoing paper.

The key result in the proof of Theorem 1.1 is a weighted Plancherel type estimate.

Next we introduce Riesz transforms, when $n=1$, associated with Bessel-Grushin operators. Let $\beta>-1 / 2$ and $a>0$. We define

$$
A_{\beta}(a)=\frac{d}{d x}+a x-\frac{\beta+1 / 2}{x} \text { and } A_{\beta}^{*}(a)=-\frac{d}{d x}+a x-\frac{\beta+1 / 2}{x}, \quad x \in(0, \infty) .
$$

Note that $A_{\beta}^{*}(a)$ is the "formal" adjoint of $A_{\beta}(a)$ in $L^{2}(0, \infty)$. We have that

$$
-B_{\beta}+a^{2} x^{2}=A_{\beta}^{*}(a) A_{\beta}(a)+2 a(\beta+1), \quad a, x \in(0, \infty) .
$$

This decomposition suggests to "formally" define the Riesz transforms for the scaled Laguerre operator $\mathfrak{L}_{\alpha}(a), \alpha=\left(\alpha_{1}, \ldots, \alpha_{m}\right) \in(-1 / 2, \infty)^{m}$ and $a>0$, as follows: for every $j=1, \ldots, m$

$$
R_{\alpha, j}(a)=A_{\alpha_{j}}(a) \mathfrak{L}_{\alpha}^{-1 / 2}(a)
$$

and

$$
\widetilde{R}_{\alpha, j}(a)=A_{\alpha_{j}}^{*}(a) \mathfrak{L}_{\alpha}^{-1 / 2}(a)
$$

According to some well-known properties of Laguerre functions (see, for instance, [13, (2.17) and (2.18), p. 1004] and [25. p. 406]) we have that, for every $k=\left(k_{1}, \ldots, k_{m}\right) \in \mathbb{N}^{m}, \alpha=\left(\alpha_{1}, \ldots, \alpha_{m}\right) \in$ $(-1 / 2, \infty)^{m}, a>0$ and $j=1, \ldots, m$,

and

$$
A_{\alpha_{j}}(a) \Phi_{k}^{\alpha}(x ; a)=-2 \sqrt{k_{j} a} \Phi_{k-e_{j}}^{\alpha+e_{j}}(x ; a), \quad x \in(0, \infty)^{m},
$$

$$
A_{\alpha_{j}}^{*}(a) \Phi_{k}^{\alpha}(x ; a)=-2 \sqrt{\left(k_{j}+1\right) a} \Phi_{k+e_{j}}^{\alpha-e_{j}}(x ; a), \quad x \in(0, \infty)^{m} .
$$

Here $e_{j}=\left(e_{j}^{1}, \ldots, e_{j}^{m}\right)$, where $e_{j}^{i}=\left\{\begin{array}{cc}0, & i \neq j \\ 1, & i=j\end{array}, j=1, \ldots, m\right.$, and we understand $\Phi_{-1}^{\beta}=0$, for every $\beta>-1 / 2$.

For every $\gamma>0$ and $\alpha \in(-1 / 2, \infty)^{m}$, the $-\gamma$-th power $\mathfrak{L}_{\alpha}^{-\gamma}(a)$ is defined by

$$
\mathfrak{L}_{\alpha}^{-\gamma}(a) f=\sum_{k \in \mathbb{N}^{m}} \frac{c_{k}^{\alpha}(a)(f)}{(2(2 \mathfrak{s}(k)+\mathfrak{s}(\alpha)+m) a)^{\gamma}} \Phi_{k}^{\alpha}(\cdot ; a), \quad f \in L^{2}\left((0, \infty)^{m}\right),
$$

where, for every $k \in \mathbb{N}^{m}$,

$$
c_{k}^{\alpha}(a)(f)=\int_{(0, \infty)^{m}} \Phi_{k}^{\alpha}(x ; a) f(x) d x, \quad f \in L^{2}\left((0, \infty)^{m}\right) .
$$


We define the Riesz transforms on $L^{2}\left((0, \infty)^{m}\right)$ (according to $[2]$ and $(3)$ ) associated with $\mathfrak{L}_{\alpha}(a)$, $\alpha \in(-1 / 2, \infty)^{m}$ and $a>0$ as follows: for every $j=1,2, \ldots, m$,

$$
R_{\alpha, j}(a) f=-2 \sum_{k \in \mathbb{N}^{m}} \sqrt{k_{j}} \frac{c_{k}^{\alpha}(a)(f)}{\sqrt{2(2 \mathfrak{s}(k)+\mathfrak{s}(\alpha)+m)}} \Phi_{k-e_{j}}^{\alpha+e_{j}}(\cdot ; a), \quad f \in L^{2}\left((0, \infty)^{m}\right),
$$

and

$$
\widetilde{R}_{\alpha, j}(a) f=-2 \sum_{k \in \mathbb{N}^{m}} \sqrt{k_{j}+1} \frac{c_{k}^{\alpha}(a)(f)}{\sqrt{2(2 \mathfrak{s}(k)+\mathfrak{s}(\alpha)+m)}} \Phi_{k+e_{j}}^{\alpha-e_{j}}(\cdot ; a), \quad f \in L^{2}\left((0, \infty)^{m}\right), \alpha_{j}>1 / 2 .
$$

Note that, in virtue of Plancherel equality for Laguerre function spaces, we deduce that, for every $j=1, \ldots, m, R_{\alpha, j}(a)$ and $\widetilde{R}_{\alpha, j}(a)$ are bounded operators from $L^{2}\left((0, \infty)^{m}\right)$ into itself. Moreover, if $f \in \operatorname{span}\left\{\Phi_{k}^{\alpha}(\cdot ; a)\right\}_{k \in \mathbb{N} m}$, the linear space generated by $\left\{\Phi_{k}^{\alpha}(\cdot ; a)\right\}_{k \in \mathbb{N}^{m}}$, then $R_{\alpha, j}(a) f=$ $A_{\alpha_{j}}(a) \mathfrak{L}_{\alpha}^{-1 / 2}(a) f$ and $\widetilde{R}_{\alpha, j}(a) f=A_{\alpha_{j}}^{*}(a) \mathfrak{L}_{\alpha}^{-1 / 2}(a) f j=1, \ldots, m . \quad L^{p}$-boundedness properties of Riesz transforms associated with Laguerre function expansions have been established in [13] and [25], among others.

The above comments suggest to define Riesz transforms $R_{\alpha}, j$ and $\widetilde{R}_{\alpha}, j$ in Bessel-Grushin settings as follows

and

$$
R_{\alpha, j}(f)(x, y)=\mathcal{F}_{2}^{-1}\left(R_{\alpha, j}(|u|)\left(\mathcal{F}_{2}(f)\right)(x, u)\right)(y), \quad f \in L^{2}\left((0, \infty)^{m} \times \mathbb{R}\right),
$$

$$
\widetilde{R}_{\alpha, j}(f)(x, y)=\mathcal{F}_{2}^{-1}\left(\widetilde{R}_{\alpha, j}(|u|)\left(\mathcal{F}_{2}(f)\right)(x, u)\right)(y), \quad f \in L^{2}\left((0, \infty)^{m} \times \mathbb{R}\right),
$$

where $\alpha \in(-1 / 2, \infty)^{m}$ and $j=1, \ldots, m$. Then, Plancherel theorem for Fourier transform implies that $R_{\alpha, j}$ and $\widetilde{R}_{\alpha, j}, j=1, \ldots, m$, are bounded operators from $L^{2}\left((0, \infty)^{m} \times \mathbb{R}^{n}\right)$ into itself.

We prove the following result.

Theorem 1.2. Let $\alpha \in[1 / 2, \infty)^{m}, j=1, \ldots, m$ and $1<p<\infty$. Then, the Riesz transforms $R_{\alpha, j}$ and $\widetilde{R}_{\alpha, j}$ are bounded operators from $L^{p}\left((0, \infty)^{m} \times \mathbb{R}\right)$ into itself.

In order to prove this theorem we start using the main idea in the proof of [16. Theorem 1.1], namely, we see $R_{\alpha, j}$ and $\widetilde{R}_{\alpha, j}, j=1, \ldots, m$, as Banach valued Fourier multipliers and then we use the celebrated Weis' multiplier result [34, Theorem 3.4]. But to show that the R-boundedness properties hold for the family of operators which appear in the Bessel-Grushin context, we can not proceed as in [16, Section 2] because Laguerre functions have not as nice operational properties as Hermite functions. Roughly speaking we take advantage that the operators we need to study are bounded perturbations of those operator handled in [16].

Throughout this paper we always denote by $c$ and $C$ positive constants that can change from one line to the other.

\section{Proof of Theorem 1.1}

The strategy of the proof of this theorem is the same as in [20] (see also [5], [19] and [28]) and the key result is a weighted Plancherel inequality. Laguerre expansions play an important role and we need to get estimations involving Laguerre functions.

The Bessel-Grushin operator $G_{\alpha}$ is selfadjoint and positive in $L^{2}\left((0, \infty)^{m} \times \mathbb{R}^{n}\right)$. Then, $-G_{\alpha}$ generates a semigroup of contractions $\left\{e^{-t G_{\alpha}}\right\}_{t>0}$ in $L^{2}\left((0, \infty)^{m} \times \mathbb{R}^{n}\right)$. Moreover, since $G_{\alpha}-G=$ $\sum_{j=1}^{m}\left(\alpha_{j}^{2}-1 / 4\right) / x_{j}^{2}$, by using the perturbation formula (see [10, Corollary 1.7, p. 161]) we get

$$
e^{-t G} f-e^{-t G_{\alpha}} f=\int_{0}^{t} e^{-(t-s) G} \sum_{j=1}^{m} \frac{\alpha_{j}^{2}-1 / 4}{x_{j}^{2}} e^{-s G_{\alpha}} f d s .
$$

Here and in the sequel, we identify each measurable function $f$ on $(0, \infty)^{m} \times \mathbb{R}^{n}$ with the function $f_{0}$ defined by

$$
f_{0}(x, y)=\left\{\begin{aligned}
f(x, y), & x \in(0, \infty)^{m}, \\
0, & x \notin \mathbb{R}^{m} \backslash\left\{(0, \infty)^{m}\right\} .
\end{aligned}\right.
$$

From (4) we deduce that $e^{-t G_{\alpha}} f \leq e^{-t G} f, 0 \leq f \in L^{2}\left((0, \infty)^{m} \times \mathbb{R}^{n}\right)$ and $t>0$.

According to [20. Proposition 3] there exists a distance $\rho$ in $\mathbb{R}^{m} \times \mathbb{R}^{n}$ such that the triple $\left(\mathbb{R}^{m} \times \mathbb{R}^{n}, \rho,|\cdot|\right)$, where $|\cdot|$ denotes the Lebesgue measure in $\mathbb{R}^{m} \times \mathbb{R}^{n}$, is a homogeneous type space (in the sense of Coifman and Weiss [7]), and that

$$
0 \leq \mathbb{W}_{t}\left(\left(x_{1}, y_{1}\right),\left(x_{2}, y_{2}\right)\right) \leq C \frac{e^{-c \rho\left(\left(x_{1}, y_{1}\right),\left(x_{2}, y_{2}\right)\right)^{2} / t}}{\left|B_{\rho}\left(\left(x_{2}, y_{2}\right), \sqrt{t}\right)\right|}, \quad\left(x_{j}, y_{j}\right) \in \mathbb{R}^{m} \times \mathbb{R}^{n}, j=1,2 \text {, and } t>0,
$$


where, for every $t>0, \mathbb{W}_{t}$ represents the integral kernel of $e^{-t G}$.

Hence, for every $t>0$, the operator $e^{-t G_{\alpha}}$ is bounded from $L^{1}\left((0, \infty)^{m} \times \mathbb{R}^{n}\right)$ into $L^{q}\left((0, \infty)^{m} \times\right.$ $\left.\mathbb{R}^{n}\right), 1 \leq q<\infty$. Then, for every $t>0$,

$$
e^{-t G_{\alpha}}(f)\left(x_{1}, y_{1}\right)=\int_{(0, \infty)^{m} \times \mathbb{R}^{n}} \mathbb{W}_{t}^{\alpha}\left(\left(x_{1}, y_{1}\right),\left(x_{2}, y_{2}\right)\right) f\left(x_{2}, y_{2}\right) d x_{2} d y_{2}, \quad f \in L^{2}\left((0, \infty)^{m} \times \mathbb{R}^{n}\right),
$$

and

$$
0 \leq \mathbb{W}_{t}^{\alpha}\left(\left(x_{1}, y_{1}\right),\left(x_{2}, y_{2}\right)\right) \leq C \frac{e^{-c \rho\left(\left(x_{1}, y_{1}\right),\left(x_{2}, y_{2}\right)\right)^{2} / t}}{\left|B_{\rho}\left(\left(x_{2}, y_{2}\right), \sqrt{t}\right)\right|}, \quad\left(x_{j}, y_{j}\right) \in(0, \infty)^{m} \times \mathbb{R}^{n}, j=1,2 \text {, and } t>0 \text {. }
$$

By defining $e^{-t G_{\alpha}}, t>0$, by (5) on $L^{p}\left((0, \infty)^{m} \times \mathbb{R}^{n}\right),\left\{e^{-t G_{\alpha}}\right\}_{t>0}$ is a bounded semigroup on $L^{p}\left((0, \infty)^{m} \times \mathbb{R}^{n}\right)$, for every $1 \leq p<\infty$.

Moreover, by [27, Proposition 1.4] the semigroup $\left\{e^{-t G_{\alpha}}\right\}_{t>0}$ is bounded and holomorphic in $L^{2}\left((0, \infty)^{m} \times \mathbb{R}^{n}\right)$ with angle $\pi / 2$. According to [27, Theorem 2.4], for every $\left(x_{j}, y_{j}\right) \in(0, \infty)^{m} \times$ $\mathbb{R}^{n}, j=1,2$, the integral kernel $\mathbb{W}_{t}^{\alpha}\left(\left(x_{1}, y_{1}\right),\left(x_{2}, y_{2}\right)\right), t>0$, can be extended to an holomorphic function $\mathbb{W}_{z}^{\alpha}\left(\left(x_{1}, y_{1}\right),\left(x_{2}, y_{2}\right)\right), \operatorname{Re}(z)>0$.

By proceeding as in the proof of [8, Lemmas 2.1 and 4.1] and [28, Lemma 3.3], for instance, we can show the following properties of the integral heat kernel $\mathbb{W}_{z}^{\alpha}, \operatorname{Re}(z)>0$.

Lemma 2.1. Let $\alpha \in[1 / 2, \infty)^{m}$. Then,

(a) For every $\left(x_{1}, y_{1}\right) \in(0, \infty)^{m} \times \mathbb{R}^{n}$ and $t, r>0$,

$$
\begin{aligned}
& \int_{\left((0, \infty)^{m} \times \mathbb{R}^{n}\right) \backslash B_{\rho}\left(\left(x_{1}, y_{1}\right), r\right)}\left|\mathbb{W}_{t}^{\alpha}\left(\left(x_{1}, y_{1}\right),\left(x_{2}, y_{2}\right)\right)\right|^{2} d x_{2} d y_{2} \leq C \frac{e^{-r^{2} / t}}{\left|B_{\rho}\left(\left(x_{1}, y_{1}\right), \sqrt{t}\right)\right|} . \\
& \text { and } \\
& \left\|\mathbb{W}_{t}^{\alpha}\left(\left(x_{1}, y_{1}\right), \cdot\right)\right\|_{L^{2}\left((0, \infty)^{m} \times \mathbb{R}^{n}\right)}^{2}=\left\|\mathbb{W}_{t}^{\alpha}\left(\cdot,\left(x_{1}, y_{1}\right)\right)\right\|_{L^{2}\left((0, \infty)^{m} \times \mathbb{R}^{n}\right)}^{2} \leq \frac{C}{\left|B_{\rho}\left(\left(x_{1}, y_{1}\right), \sqrt{t}\right)\right|} .
\end{aligned}
$$

(b) For every $s>0, \mu \in \mathbb{R}, R>0$ and $\left(x_{1}, y_{1}\right) \in(0, \infty)^{m} \times \mathbb{R}^{n}$,

$$
\int_{(0, \infty)^{m} \times \mathbb{R}^{n}}\left|\mathbb{W}_{(1+i \mu) R^{-2}}^{\alpha}\left(\left(x_{1}, y_{1}\right),\left(x_{2}, y_{2}\right)\right)\right|^{2}\left[\rho\left(\left(x_{1}, y_{1}\right),\left(x_{2}, y_{2}\right)\right)\right]^{s} d x_{2} d y_{2} \leq C \frac{R^{-s}(1+|\mu|)^{s}}{\left|B_{\rho}\left(\left(x_{1}, y_{1}\right), 1 / R\right)\right|} \text {. }
$$

Let $R>0$. As it was commented above the operator $e^{-R^{-2} G_{\alpha}}$ is bounded from $L^{1}\left((0, \infty)^{m} \times \mathbb{R}^{n}\right)$ into $L^{2}\left((0, \infty)^{m} \times \mathbb{R}^{n}\right)$. Suppose that $F$ is a bounded measurable function on $\mathbb{R}$ such that supp $F \subset$ $\left[0, R^{2}\right]$. We define $H_{2}(\lambda)=e^{-\lambda R^{-2}}, \lambda \in \mathbb{R}$, and $H_{1}=F / H_{2}$. It is clear that $H_{2}\left(G_{\alpha}\right)=e^{-R^{-2} G_{\alpha}}$ and that $H_{1}\left(G_{\alpha}\right)$ is a bounded operator from $L^{2}\left((0, \infty)^{m} \times \mathbb{R}^{n}\right)$ into itself with

$$
\left\|H_{1}\left(G_{\alpha}\right)\right\|_{L^{2}\left((0, \infty)^{m} \times \mathbb{R}^{n}\right) \rightarrow L^{2}\left((0, \infty)^{m} \times \mathbb{R}^{n}\right)} \leq\left\|H_{1}\right\|_{L^{\infty}(0, \infty)} \leq e\|F\|_{L^{\infty}(0, \infty)} .
$$

Hence, the operator $F\left(G_{\alpha}\right)$ is associated to the kernel

$$
K_{F\left(G_{\alpha}\right)}\left(\left(x_{1}, y_{1}\right),\left(x_{2}, y_{2}\right)\right)=H_{1}\left(G_{\alpha}\right)\left[\mathbb{W}_{R^{-2}}^{\alpha}\left(\cdot,\left(x_{2}, y_{2}\right)\right)\right]\left(x_{1}, y_{1}\right), \quad\left(x_{j}, y_{j}\right) \in(0, \infty)^{m} \times \mathbb{R}^{n}, j=1,2 .
$$

Then, Lemma 2.1. (a), leads to

$$
\left\|K_{F\left(G_{\alpha}\right)}\left(\left(x_{1}, y_{1}\right), \cdot\right)\right\|_{L^{2}\left((0, \infty)^{m} \times \mathbb{R}^{n}\right)}^{2} \leq C \frac{\|F\|_{L^{\infty}(0, \infty)}^{2}}{\left|B_{\rho}\left(\left(x_{1}, y_{1}\right), 1 / R\right)\right|} .
$$

The arguments presented in the proof of [28, Lemma 3.5] (see also [8, Lemma 4.3, (a)]) allow us to establish the following result.

Lemma 2.2. Let $\alpha \in(-1 / 2, \infty)^{m}$ and $R, s>0$. For every $\varepsilon>0$ there exists $C_{\varepsilon}>0$ such that

$$
\int_{(0, \infty)^{m} \times \mathbb{R}^{n}}\left|K_{F\left(G_{\alpha}\right)}\left(\left(x_{1}, y_{1}\right),\left(x_{2}, y_{2}\right)\right)\right|^{2}\left[1+R \rho\left(\left(x_{1}, y_{1}\right),\left(x_{2}, y_{2}\right)\right)\right]^{s} d x_{2} d y_{2} \leq C_{\varepsilon} \frac{\left\|\delta_{R^{2}} F\right\|_{W_{s / 2+\varepsilon}^{\infty}}^{2}}{\left|B_{\rho}\left(\left(x_{1}, y_{1}\right), 1 / R\right)\right|},
$$

for every bounded measurable function $F$ such that supp $F \subset\left[0, R^{2}\right]$.

We now define, for $j=1, \ldots, m$ and $l=1, \ldots, n$, the operators

$$
M_{j} f(x, y)=x_{j} f(x, y), \quad f \in D\left(M_{j}\right)=\left\{g \in L^{2}\left((0, \infty)^{m} \times \mathbb{R}^{n}\right), \quad x_{j} g \in L^{2}\left((0, \infty)^{m} \times \mathbb{R}^{n}\right)\right\},
$$

$\mathcal{D}_{l} f=\mathcal{F}_{2}^{-1}\left(-i u_{l} \mathcal{F}_{2}(f)(u)\right), \quad f \in D\left(\mathcal{D}_{l}\right)=\left\{g \in L^{2}\left((0, \infty)^{m} \times \mathbb{R}^{n}\right), \quad u_{l} \mathcal{F}_{2}(g) \in L^{2}\left((0, \infty)^{m} \times \mathbb{R}^{n}\right)\right\}$ 
As it was done in [20], we denote by $|M|$ the operator of multiplication by $|x|$, and $|\mathcal{D}|$ represents the operator $\left(-\sum_{j=1}^{n} \frac{\partial^{2}}{\partial y_{j}^{2}}\right)^{1 / 2}$.

It is clear that $|M|$ is a positive and selfadjoint operator. Moreover, we have that

$$
|\mathcal{D}| f=\mathcal{F}_{2}^{-1}\left(|u| \mathcal{F}_{2}(f)(u)\right), \quad f \in D(|\mathcal{D}|) .
$$

Then, $|\mathcal{D}|^{d} f=\mathcal{F}_{2}^{-1}\left(|u|^{d} \mathcal{F}_{2}(f)(u)\right), f \in D\left(|\mathcal{D}|^{d}\right)$, and $d \in \mathbb{N}$. Also we define the operator $\mathcal{S}$ by

$$
\mathcal{S} f(x, y)=|x| \mathcal{F}_{2}^{-1}\left(|u| \mathcal{F}_{2}(f(x, \cdot))(u)\right)(y),
$$

for every $f \in D(\mathcal{S})=\left\{f \in L^{2}\left((0, \infty)^{m} \times \mathbb{R}^{n}\right):|x||u| \mathcal{F}_{2}(f(x, \cdot))(u) \in L^{2}\left((0, \infty)^{m} \times \mathbb{R}^{n}\right)\right\}$. Note that $D(|M||\mathcal{D}|) \subsetneq D(\mathcal{S})$.

Next result is a version of [20, Proposition 4] in our setting.

Lemma 2.3. Let $\alpha \in(-1 / 2, \infty)^{m}$ and $\gamma>0$. Then,

$$
\left\||M|^{\gamma} f\right\|_{L^{2}\left((0, \infty)^{m} \times \mathbb{R}^{n}\right)} \leq C\left\|G_{\alpha}^{\gamma / 2}|\mathcal{D}|^{-\gamma} f\right\|_{L^{2}\left((0, \infty)^{m} \times \mathbb{R}^{n}\right)}
$$

for every $f \in \operatorname{Ran}\left(|\mathcal{D}|^{\gamma}\right)$, the range of $|\mathcal{D}|^{\gamma}$, such that $|\mathcal{D}|^{-\gamma} f \in D\left(G_{\alpha}^{\gamma / 2}\right)$.

Proof. Our first objective is to show that, for every $d \in \mathbb{N}$,

$$
\left\|\mathcal{S}^{2 d} f\right\|_{L^{2}\left((0, \infty)^{m} \times \mathbb{R}^{n}\right)} \leq C\left\|G_{\alpha}^{d} f\right\|_{L^{2}\left((0, \infty)^{m} \times \mathbb{R}^{n}\right)}, \quad f \in D\left(G_{\alpha}^{d}\right) .
$$

We consider the operator

$$
\mathcal{A}_{\alpha}(f)(x, y)=\mathcal{F}_{2}^{-1}\left[\sum_{k \in \mathbb{N}^{m}} \frac{c_{k}^{\alpha}\left(\mathcal{F}_{2}(f) ; u\right)}{2(2 \mathfrak{s}(k)+\mathfrak{s}(\alpha)+m)|u|} \Phi_{k}^{\alpha}(x ;|u|)\right](y), \quad f \in D\left(\mathcal{A}_{\alpha}\right),
$$

being

$$
D\left(\mathcal{A}_{\alpha}\right)=\left\{g \in L^{2}\left((0, \infty)^{m} \times \mathbb{R}^{n}\right): \sum_{k \in \mathbb{N}^{m}} \frac{c_{k}^{\alpha}\left(\mathcal{F}_{2}(f) ; u\right)}{2(2 \mathfrak{s}(k)+\mathfrak{s}(\alpha)+m)|u|} \Phi_{k}^{\alpha}(x ;|u|) \in L^{2}\left((0, \infty)^{m} \times \mathbb{R}^{n}\right)\right\} .
$$

Observe that, for every $f \in D\left(\mathcal{A}_{\alpha}\right), \mathcal{A}_{\alpha} f \in D\left(G_{\alpha}\right)$ and $G_{\alpha} \mathcal{A}_{\alpha} f=f$. Furthermore, if $f \in D\left(G_{\alpha}\right)$, $G_{\alpha} f \in D\left(\mathcal{A}_{\alpha}\right)$ and $\mathcal{A}_{\alpha} G_{\alpha} f=f$.

We treat the case $d=1$. The inequality in (7) is now equivalent to the following one

$$
\left\|\mathcal{S}^{2} \mathcal{A}_{\alpha} f\right\|_{L^{2}\left((0, \infty)^{m} \times \mathbb{R}^{n}\right)} \leq C\|f\|_{L^{2}\left((0, \infty)^{m} \times \mathbb{R}^{n}\right)}, \quad f \in D\left(\mathcal{A}_{\alpha}\right) .
$$

According to Plancherel equality for the Fourier transform, (8) holds if, and only if, $D\left(G_{\alpha}\right) \subseteq$ $D\left(\mathcal{S}^{2}\right)=\left\{f \in L^{2}\left((0, \infty)^{m} \times \mathbb{R}^{n}\right):|x|^{2}|u|^{2} \mathcal{F}_{2}(f(x, \cdot))(u) \in L^{2}\left((0, \infty)^{m} \times \mathbb{R}^{n}\right)\right\}$ and the operator $T_{\alpha}$ defined by

$$
T_{\alpha}(f)(x, u)=\sum_{k \in \mathbb{N}^{m}} \frac{|x|^{2}|u| c_{k}^{\alpha}\left(\mathcal{F}_{2}(f) ; u\right)}{2(2 \mathfrak{s}(k)+\mathfrak{s}(\alpha)+m)} \Phi_{k}^{\alpha}(x ;|u|), \quad f \in L^{2}\left((0, \infty)^{m} \times \mathbb{R}^{n}\right)
$$

is bounded from $L^{2}\left((0, \infty)^{m} \times \mathbb{R}^{n}\right)$ into itself.

In order to show the $L^{2}$-boundedness property for $T_{\alpha}$ we consider the operator $\mathfrak{L}_{\alpha}^{-1}$ defined by

$$
\mathfrak{L}_{\alpha}^{-1} g=\sum_{k \in \mathbb{N}^{m}} \frac{c_{k}^{\alpha}(g)}{2(2 \mathfrak{s}(k)+\mathfrak{s}(\alpha)+m)} \Phi_{k}^{\alpha}, \quad g \in L^{2}\left((0, \infty)^{m}\right),
$$

where

$$
c_{k}^{\alpha}(g)=\int_{(0, \infty)^{m}} \varphi_{k}^{\alpha}(x) g(x) d x, \quad k \in \mathbb{N}^{m} .
$$

The operator $|x|^{2} \mathfrak{L}_{\alpha}^{-1}$ is bounded from $L^{2}\left((0, \infty)^{m}\right)$ into itself. Indeed, let $g \in L^{2}\left((0, \infty)^{m}\right)$. We define the function $g_{0}$ by

$$
g_{0}(x)=g(x), x \in(0, \infty)^{m}, \quad \text { and } \quad g_{0}(x)=0, x \in \mathbb{R}^{m} \backslash\left\{(0, \infty)^{m}\right\} .
$$


We have that $\mathfrak{L}_{\alpha}^{-1}(|g|)(x) \leq C H^{-1}\left(\left|g_{0}\right|\right), x \in(0, \infty)^{m}$, where $H$ represents the Hermite operator. Then, the $L^{2}$-boundedness of the operator $|x|^{2} \mathfrak{L}_{\alpha}^{-1}$ follows from [3, Lemma 3] (see also [4]). We get

$$
\begin{aligned}
\left\|T_{\alpha}(f)\right\|_{L^{2}\left((0, \infty)^{m} \times \mathbb{R}^{n}\right)}^{2} & =\int_{\mathbb{R}^{n}} \int_{(0, \infty)^{m}}\left|\sum_{k \in \mathbb{N}^{m}} \frac{|x|^{2}|u| c_{k}^{\alpha}\left(\mathcal{F}_{2}(f) ; u\right)}{2(2 \mathfrak{s}(k)+\mathfrak{s}(\alpha)+m)} \Phi_{k}^{\alpha}(x ;|u|)\right|^{2} d x d u \\
& =\int_{\mathbb{R}^{n}} \int_{(0, \infty)^{m}}\left|\sum_{k \in \mathbb{N}^{m}} \frac{|x|^{2} c_{k}^{\alpha}\left(\mathcal{F}_{2}(f)(y / \sqrt{|u|}, u)\right)}{2(2 \mathfrak{s}(k)+\mathfrak{s}(\alpha)+m)} \Phi_{k}^{\alpha}(x)\right|^{2} d x \frac{d u}{|u|^{m / 2}} \\
& =\left.\left.\int_{\mathbb{R}^{n}} \int_{(0, \infty)^{m}}|| x\right|^{2} \mathfrak{L}_{\alpha}^{-1}\left(\mathcal{F}_{2}(f)\left(\frac{y}{\sqrt{|u|}}, u\right)\right)(x)\right|^{2} d x \frac{d u}{|u|^{m / 2}} \\
& \leq C \int_{\mathbb{R}^{n}} \int_{(0, \infty)^{m}}\left|\mathcal{F}_{2}(f)\left(\frac{y}{\sqrt{|u|}}, u\right)\right|^{2} d y \frac{d u}{|u|^{m / 2}} \\
& \leq C \|_{L^{2}\left((0, \infty)^{m} \times \mathbb{R}^{n}\right)}^{2}, \quad f \in L^{2}\left((0, \infty)^{m} \times \mathbb{R}^{n}\right) .
\end{aligned}
$$

Note that $\mathcal{F}_{2}(f)(\cdot / \sqrt{|u|}, u) \in L^{2}\left((0, \infty)^{m}\right)$, a.e. $u \in \mathbb{R}^{n}$, and then the coefficient $c_{k}^{\alpha}$ in the second equality above, which is given by $(9)$, is understood as a function of $u$. On the other hand, the property $D\left(G_{\alpha}\right) \subseteq D\left(\mathcal{S}^{2}\right)$ can be also deduced from the previous argument.

An inductive procedure allows to show that (7) is true for every $d \in \mathbb{N}$. The imaginary powers of the operators $\mathcal{S}$ and $G_{\alpha}$ are bounded in $L^{2}\left((0, \infty)^{m} \times \mathbb{R}^{n}\right)$ (see [21, p. 640] or [35, Theorem B]). By using [18, Theorem 11.6.1] and [1, Theorem 4.1.2] we get that there exists $C>0$ such that

$$
\left\|\mathcal{S}^{\gamma} f\right\|_{L^{2}\left((0, \infty)^{m} \times \mathbb{R}^{n}\right)} \leq C\left(\|f\|_{L^{2}\left((0, \infty)^{m} \times \mathbb{R}^{n}\right)}+\left\|G_{\alpha}^{\gamma / 2} f\right\|_{L^{2}\left((0, \infty)^{m} \times \mathbb{R}^{n}\right)}\right), \quad f \in D\left(G_{\alpha}^{\gamma / 2}\right) .
$$

In the usual way, the homogeneity allows us to obtain that

$$
\left\|\mathcal{S}^{\gamma} f\right\|_{L^{2}\left((0, \infty)^{m} \times \mathbb{R}^{n}\right)} \leq C\left\|G_{\alpha}^{\gamma / 2} f\right\|_{L^{2}\left((0, \infty)^{m} \times \mathbb{R}^{n}\right)}, \quad f \in D\left(G_{\alpha}^{\gamma / 2}\right) .
$$

Since $|\mathcal{D}|^{\gamma}$ is an one to one operator, we deduce that (6) holds.

Lemma 2.4. Suppose that $H$ is a compactly supported Borel measurable complex function defined on $\mathbb{R}$. For every $f \in C_{c}^{\infty}\left((0, \infty)^{m}\right) \otimes C_{c}^{\infty}\left(\mathbb{R}^{n}\right)$, we have that

$$
H\left(G_{\alpha}\right) f(x, t)=\int_{\mathbb{R}^{n}} \int_{(0, \infty)^{m}} K_{H}^{\alpha}(y, z ; x, t) f(y, z) d y d z, \quad x \in(0, \infty)^{m} \text { and } t \in \mathbb{R}^{n},
$$

being, for $x, y \in(0, \infty)^{m}$ and $z, t \in \mathbb{R}^{n}$,

$$
K_{H}^{\alpha}(y, z ; x, t)=\frac{1}{(2 \pi)^{n}} \int_{\mathbb{R}^{n}} \sum_{k \in \mathbb{N}^{m}} H(2(2 \mathfrak{s}(k)+\mathfrak{s}(\alpha)+m)|u|) \Phi_{k}^{\alpha}(x ;|u|) \Phi_{k}^{\alpha}(y ;|u|) e^{-i u \cdot(z-t)} d u .
$$

Moreover, for $y \in(0, \infty)^{m}$ and $z \in \mathbb{R}^{n}$,

$$
\left\|K_{H}^{\alpha}(y, z ; \cdot, \cdot)\right\|_{L^{2}\left((0, \infty)^{m} \times \mathbb{R}^{n}\right)}^{2}=\int_{\mathbb{R}^{n}} \sum_{k \in \mathbb{N}^{m}}\left|H(2(2 \mathfrak{s}(k)+\mathfrak{s}(\alpha)+m)|u|) \Phi_{k}^{\alpha}(y ;|u|)\right|^{2} d u .
$$

Proof. We consider $f(x, t)=h(x) g(t)$, where $h \in C_{c}^{\infty}\left((0, \infty)^{m}\right)$ and $g \in C_{c}^{\infty}\left(\mathbb{R}^{n}\right)$. We can write

$$
\begin{aligned}
& H\left(G_{\alpha}\right) f(x, t)=\mathcal{F}_{2}^{-1}\left[\sum_{k \in \mathbb{N}^{m}} H(2(2 \mathfrak{s}(k)+\mathfrak{s}(\alpha)+m)|u|)\right. \\
&\left.\quad \times \int_{(0, \infty)^{m}} \Phi_{k}^{\alpha}(y ;|u|) h(y) d y \mathcal{F}_{2}(g)(u) \Phi_{k}^{\alpha}(x ;|u|)\right](t) \\
&=\frac{1}{(2 \pi)^{n}} \int_{\mathbb{R}^{n}} e^{i u \cdot t} \int_{(0, \infty)^{m}} \sum_{k \in \mathbb{N}^{m}} H(2(2 \mathfrak{s}(k)+\mathfrak{s}(\alpha)+m)|u|) \Phi_{k}^{\alpha}(x ;|u|) \Phi_{k}^{\alpha}(y ;|u|) h(y) d y \\
& \quad \times \int_{\mathbb{R}^{n}} e^{-i u \cdot z} g(z) d z d u \\
&=\int_{\mathbb{R}^{n}} \int_{(0, \infty)^{m}} K_{H}^{\alpha}(y, z ; x, t) h(y) g(z) d y d z, \quad x \in(0, \infty)^{m} \text { and } t \in \mathbb{R}^{n} .
\end{aligned}
$$

Indeed, the interchange of the order of integration can be justified as follows. Since $H$ has bounded support there exists $b>0$ such that $H(2(2 \mathfrak{s}(k)+\mathfrak{s}(\alpha)+m)|u|)=0$, provided that $2(2 \mathfrak{s}(k)+\mathfrak{s}(\alpha)+$ 
$m)|u|>b, u \in \mathbb{R}^{n}$ and $k \in \mathbb{N}^{m}$. Then, $H(2(2 \mathfrak{s}(k)+\mathfrak{s}(\alpha)+m)|u|)=0$, when $2(\mathfrak{s}(\alpha)+m)|u|>b$, $u \in \mathbb{R}^{n}$ and $k \in \mathbb{N}^{m}$. Hence, we have that

$$
\begin{aligned}
\int_{\mathbb{R}^{n}} \int_{(0, \infty)^{m}} \int_{\mathbb{R}^{n}}|g(z)||h(y)| \sum_{k \in \mathbb{N}^{m}}|H(2(2 \mathfrak{s}(k)+\mathfrak{s}(\alpha)+m)|u|)|\left|\Phi_{k}^{\alpha}(x ;|u|)\right|\left|\Phi_{k}^{\alpha}(y ;|u|)\right| d z d y d u \\
\leq C \int_{B(0, b /(2 \mathfrak{s}(\alpha)+2 m))} \int_{(0, \infty)^{m}} \int_{\mathbb{R}^{n}}|g(z)||h(y)| \\
\quad \times \sum_{k \in \mathbb{N}^{m}, 2 \mathfrak{s}(k)+\mathfrak{s}(\alpha)+m<b /(2|u|)}\left|\Phi_{k}^{\alpha}(x ;|u|)\right|\left|\Phi_{k}^{\alpha}(y ;|u|)\right| d z d y d u
\end{aligned}
$$

Furthermore, since $\left|\Phi_{k}^{\alpha}(z)\right| \leq C, k \in \mathbb{N}^{m}$ and $z \in(0, \infty)^{m}$, for a certain $C>0$ (see [24, (27)], we get

$$
\begin{aligned}
& \int_{B(0, b /(2 \mathfrak{s}(\alpha)+2 m))} \int_{(0, \infty)^{m}} \int_{\mathbb{R}^{n}}|g(z) \| h(y)| \sum_{k \in \mathbb{N}^{m}, 2 \mathfrak{s}(k)+\mathfrak{s}(\alpha)+m<b /(2|u|)}\left|\Phi_{k}^{\alpha}(x ;|u|)\right|\left|\Phi_{k}^{\alpha}(y ;|u|)\right| d z d y d u \\
& \quad \leq C\|g\|_{L^{1}\left(\mathbb{R}^{n}\right)} \int_{(0, \infty)^{m}}|h(y)| \int_{B(0, b /(2 \mathfrak{s}(\alpha)+2 m))} \sum_{k \in \mathbb{N}^{m}, 2 \mathfrak{s}(k)+\mathfrak{s}(\alpha)+m<b /(2|u|)}|u|^{\frac{m}{2}} d y d u \\
& \quad \leq C\|g\|_{L^{1}\left(\mathbb{R}^{n}\right)} \int_{(0, \infty)^{m}}|h(y)| \int_{B(0, b /(2 \mathfrak{s}(\alpha)+2 m))} \frac{|u|^{\frac{m}{2}}}{|u|^{m}} d y d u \\
& \leq C\|g\|_{L^{1}\left(\mathbb{R}^{n}\right)}\|h\|_{L^{1}\left((0, \infty)^{m}\right)}<\infty .
\end{aligned}
$$

We have made the change of variable $|u|^{1 / 2} y=Y$ and have taken into account that $\sharp\left\{k \in \mathbb{N}^{m}\right.$ : $\mathfrak{s}(k) \leq \ell\} \leq C \ell^{m}$, where $\sharp A$ represents the cardinal of $A$.

On the other hand, by using Plancherel equality for Fourier transforms and Laguerre expansions we easily obtain 10 .

For every $R>0$, we define the weight function,

$$
w_{R}((x, t),(y, z))=\min \{R, 1 /|y|\}|x|, \quad x, y \in(0, \infty)^{m} \text { and } t, z \in \mathbb{R}^{n} .
$$

The following is our crucial weighted Plancherel inequality.

Lemma 2.5. Assume that $\gamma \in[0, n / 2)$ and $H$ is a compactly supported Borel measurable complex function defined on $\mathbb{R}$. Then, for $y \in(0, \infty)^{m}$ and $z \in \mathbb{R}^{n}$,

$$
\left\||M|^{\gamma} K_{H}^{\alpha}(y, z ; \cdot, \cdot)\right\|_{L^{2}\left((0, \infty)^{m} \times \mathbb{R}^{n}\right)}^{2} \leq C \int_{0}^{\infty}|H(\omega)|^{2} \omega^{(n+m) / 2} \min \left\{\omega^{-\gamma+n / 2},|y|^{2 \gamma-n}\right\} \frac{d \omega}{\omega} .
$$

Particulary, when supp $H \subset\left[R^{2}, 4 R^{2}\right]$, for some $R>0$, we have that

$$
\sup _{(y, z) \in(0, \infty)^{m} \times \mathbb{R}^{n}}\left|B_{\rho}((y, z), 1 / R)\right|^{1 / 2}\left\|w_{R}((\cdot, \cdot),(y, z))^{\gamma} K_{H}^{\alpha}(y, z ; \cdot, \cdot)\right\|_{L^{2}\left((0, \infty)^{m} \times \mathbb{R}^{n}\right)} \leq C\left\|\delta_{R^{2}} H\right\|_{L^{2}(\mathbb{R})},
$$

being $C>0$ independent on $R$.

Proof. We define, for every $\ell \in \mathbb{N}$,

$$
H_{\ell}(\omega)=\chi_{(1 / \ell, \ell)}(\omega) H(\omega), \quad \omega \in \mathbb{R} .
$$

By using monotone convergence theorem it follows that

$$
\left\|K_{H}^{\alpha}(y, z ; \cdot, \cdot)\right\|_{L^{2}\left((0, \infty)^{m} \times \mathbb{R}^{n}\right)}=\lim _{\ell \rightarrow \infty}\left\|K_{H_{\ell}}^{\alpha}(y, z ; \cdot, \cdot)\right\|_{L^{2}\left((0, \infty)^{m} \times \mathbb{R}^{n}\right)}, \quad y \in(0, \infty)^{m} \text { and } z \in \mathbb{R}^{n} .
$$

Let $\ell \in \mathbb{N}$. Note that

$$
\int_{\mathbb{R}^{n}} \sum_{k \in \mathbb{N}^{m}}\left|H_{\ell}(2(2 \mathfrak{s}(k)+\mathfrak{s}(\alpha)+m)|u|) \Phi_{k}^{\alpha}(y ;|u|)\right|^{2} \frac{(2(2 \mathfrak{s}(k)+\mathfrak{s}(\alpha)+m)|u|)^{\gamma}}{|u|^{2 \gamma}} d u<\infty
$$

if, and only if,

$$
\int_{\mathbb{R}^{n}} \sum_{k \in \mathbb{N}^{m}}\left|H_{\ell}(2(2 \mathfrak{s}(k)+\mathfrak{s}(\alpha)+m)|u|) \Phi_{k}^{\alpha}(y ;|u|)\right|^{2} \frac{d u}{|u|^{2 \gamma}}<\infty .
$$

Our next objective is to estimate the following function, for each $y \in(0, \infty)^{m}$

$$
\Lambda_{\ell}(y)=\int_{\mathbb{R}^{n}} \sum_{k \in \mathbb{N}^{m}}\left|H_{\ell}(2(2 \mathfrak{s}(k)+\mathfrak{s}(\alpha)+m)|u|) \Phi_{k}^{\alpha}(y ;|u|)\right|^{2} \frac{(2(2 \mathfrak{s}(k)+\mathfrak{s}(\alpha)+m)|u|)^{\gamma}}{|u|^{2 \gamma}} d u .
$$


By making straightforward manipulations we get, for each $y \in(0, \infty)^{m}$

$$
\Lambda_{\ell}(y) \leq C \int_{0}^{\infty}\left|H_{\ell}(\omega)\right|^{2} \sum_{k \in \mathbb{N}^{m}}\left|\Phi_{k}^{\alpha}\left(\frac{\sqrt{\omega} y}{\sqrt{2(2 \mathfrak{s}(k)+\mathfrak{s}(\alpha)+m)}}\right)\right|^{2} \frac{\omega^{n+m / 2-1-\gamma}}{(2 \mathfrak{s}(k)+\mathfrak{s}(\alpha)+m)^{n+m / 2-2 \gamma}} d \omega .
$$

According to [23, p. 1124], there exist $C, \eta, \lambda$ and $\xi \in(0, \infty)$ such that

$$
\left|\varphi_{k}^{\alpha}(x)\right| \leq C \mathcal{M}_{k}^{\alpha}(x), \quad x \in(0, \infty), \alpha>-1 / 2, \text { and } k \in \mathbb{N},
$$

where

$$
\mathcal{M}_{k}^{\alpha}(x)=x^{\alpha+1 / 2}\left(\frac{1}{\nu_{k}}+x^{2}\right)^{-1 / 4-\alpha / 2}\left(\nu_{k}^{1 / 3}+\left|x^{2}-\nu_{k}\right|\right)^{-1 / 4} \Psi_{k}^{\alpha}(x), \quad x \in(0, \infty),
$$

and

$$
\Psi_{k}^{\alpha}(x)= \begin{cases}1, & 0 \leq x^{2} \leq \nu_{k}, \\ \exp \left(-\eta\left|\nu_{k}-x^{2}\right|^{3 / 2} / \nu_{k}^{1 / 2}\right), & \nu_{k} \leq x^{2} \leq(1+\lambda) \nu_{k}, \\ e^{-\xi x^{2}}, & (1+\lambda) \nu_{k} \leq x^{2},\end{cases}
$$

being $\nu_{k}=4 k+2 \alpha+2, k \in \mathbb{N}$.

Then, we deduce that

$$
\left|\varphi_{k}^{\alpha}(x)\right| \leq C \begin{cases}\left(\nu_{k}^{1 / 3}+\left|x^{2}-\nu_{k}\right|\right)^{-1 / 4}, & x \in(0, \infty) \\ e^{-\xi x^{2}}, & x^{2} \geq(1+\lambda) \nu_{k}\end{cases}
$$

Furthermore, as it was done in [20, Lemma 8], one can get for all $k \in \mathbb{N}^{m}$ and $\alpha \in(-1 / 2, \infty)^{m}$

$$
\left|\Phi_{k}^{\alpha}(x)\right| \leq C \begin{cases}\nu_{k}^{m / 2-1}, & x \in(0, \infty)^{m} \\ e^{-\xi|x|^{2}}, & |x|^{2} \geq(1+\lambda) \nu_{k}\end{cases}
$$

where, in this case, $\nu_{k}=2(2 \mathfrak{s}(k)+\mathfrak{s}(\alpha)+m)$. By proceeding as in the proof of [20, Lemma 9], 13] and (14) allows us to obtain that, for every $\varepsilon>0$,

$$
\sup _{x \in(0, \infty)^{m}} \sum_{k \in \mathbb{N}^{m}} \frac{\max \{1,|x|\}^{\varepsilon}}{(2 \mathfrak{s}(k)+\mathfrak{s}(\alpha)+m)^{\varepsilon+m / 2}}\left|\varphi_{k}^{\alpha}\left(\frac{x}{\sqrt{2(2 \mathfrak{s}(k)+\mathfrak{s}(\alpha)+m)}}\right)\right|^{2}<\infty .
$$

From 15 we deduce that

$$
\Lambda_{\ell}(y) \leq C \int_{0}^{\infty}\left|H_{\ell}(\omega)\right|^{2} \omega^{(n+m) / 2} \min \left\{\omega^{-\gamma+n / 2},|y|^{2 \gamma-n}\right\} \frac{d \omega}{\omega}, \quad y \in(0, \infty)^{m},
$$

provided that $\gamma \in[0, n / 2)$.

By Lemma 2.3, we get, for every $y \in(0, \infty)^{m}$ and $z \in \mathbb{R}^{n}$,

$$
\left\||M|^{\gamma} K_{H_{\ell}}^{\alpha}(y, z ; \cdot, \cdot)\right\|_{L^{2}((0, \infty) \times \mathbb{R})} \leq C \int_{0}^{\infty}\left|H_{\ell}(\omega)\right|^{2} \omega^{(n+m) / 2} \min \left\{\omega^{-\gamma+n / 2},|y|^{2 \gamma-n}\right\} \frac{d \omega}{\omega} .
$$

where $C$ does not depend on $\ell$. By taking limits as $\ell \rightarrow \infty$ we obtain (11).

Suppose now that supp $H \subset\left[R^{2}, 4 R^{2}\right]$, where $R>0$. It is clear that

$$
\begin{aligned}
\int_{0}^{\infty}|H(\omega)|^{2} \omega^{(n+m) / 2} \min \left\{\omega^{-\gamma+n / 2},|y|^{2 \gamma-n}\right\} \frac{d \omega}{\omega} \\
\quad=\int_{1}^{4}\left|H\left(R^{2} v\right)\right|^{2} R^{n+m} v^{(n+m) / 2-1} \min \left\{R^{-2 \gamma+n} v^{-\gamma+n / 2},|y|^{2 \gamma-n}\right\} d v \\
\leq C R^{n+m} \min \left\{R^{n-2 \gamma},|y|^{2 \gamma-n}\right\} \int_{1}^{4}\left|H\left(R^{2} v\right)\right|^{2} v^{(n+m) / 2-1} d v, \quad y \in(0, \infty)^{m} .
\end{aligned}
$$

Since $\left|B_{\rho}((x, t), R)\right| \sim R^{m+n} \max \{|x|, R\}^{n}, x \in(0, \infty)^{m}, R>0$ and $t \in \mathbb{R}^{n}$ ([20, Proposition 3, $(9)]$ ), we deduce 12 .

The proof of Theorem 1.1] can be finished now by proceeding as in [20, Section 4] (see also [5. Section 4]). 


\section{Proof of Theorem 1.2}

3.1. Some definitions and estimates. Let $\beta \geq 1 / 2$. The Laguerre operator $-L_{\beta}$ generates the semigroup of contractions $\left\{W_{t}^{\beta}\right\}_{t>0}$ in $L^{2}(0, \infty)$, where, for every $t>0$,

$$
W_{t}^{\beta}(g)=\sum_{k=0}^{\infty} e^{-2 t(2 k+\beta+1)} c_{k}^{\beta}(g) \varphi_{k}^{\beta}, \quad g \in L^{2}(0, \infty),
$$

where, for every $k \in \mathbb{N}$,

$$
c_{k}^{\beta}(g)=\int_{0}^{\infty} \varphi_{k}^{\beta}(y) g(y) d y .
$$

According to the Mehler's formula [32, (1.1.47)], for every $t>0$, we can write

$$
W_{t}^{\beta}(g)(x)=\int_{0}^{\infty} W_{t}^{\beta}(x, y) g(y) d y, \quad x \in(0, \infty),
$$

for every $g \in L^{2}(0, \infty)$, where, for every $t, x, y \in(0, \infty)$

$$
W_{t}^{\beta}(x, y)=\left(\frac{2 e^{-2 t}}{1-e^{-4 t}}\right)^{1 / 2}\left(\frac{2 x y e^{-2 t}}{1-e^{-4 t}}\right)^{1 / 2} I_{\beta}\left(\frac{2 x y e^{-2 t}}{1-e^{-4 t}}\right) \exp \left(-\frac{1}{2}\left(x^{2}+y^{2}\right) \frac{1+e^{-4 t}}{1-e^{-4 t}}\right) .
$$

To simplify notation, it is convenient to consider the functions

Observe that

$$
\mathfrak{a}(t)=\frac{2 e^{-2 t}}{1-e^{-4 t}} \quad \text { and } \quad \mathfrak{b}(t)=\frac{1}{2} \frac{1+e^{-4 t}}{1-e^{-4 t}}, \quad t>0 .
$$

$$
\mathfrak{a}(t) t \leq 1 / 2, \quad \mathfrak{b}(t) t \geq 1 / 4 \quad \text { and } \quad|1-2 \mathfrak{b}(t)| \leq \mathfrak{a}(t), \quad t \in(0, \infty),
$$

and these bounds will be used repeatedly.

Here $I_{\beta}$ denotes the modified Bessel function of the first kind and order $\beta$. By defining $W_{t}^{\beta}$, $t>0$, in $L^{p}(0, \infty), 1 \leq p \leq \infty$, by the integral in $(16),\left\{W_{t}^{\beta}\right\}_{t>0}$ is a semigroup of contractions in $L^{p}(0, \infty), 1 \leq p \leq \infty$ (see [26, Theorem 4.1]).

In the sequel we will use the following properties of the Bessel function $I_{\nu}, \nu>-1 / 2$. By [17. (5.11.8) and (5.16.4)], for every $n \in \mathbb{N}$,

$$
\sqrt{2 \pi z} I_{\nu}(z) e^{-z}=\sum_{r=0}^{n}(-1)^{r} \frac{[\nu, r]}{(2 z)^{r}}+\mathcal{O}\left(\frac{1}{z^{n+1}}\right), \quad z \in(0, \infty)
$$

where $[\nu, 0]=1$ and

$$
[\nu, r]=\frac{\left(4 \nu^{2}-1\right)\left(4 \nu^{2}-3^{2}\right) \cdots\left(4 \nu^{2}-(2 r-1)^{2}\right)}{2^{2 r} \Gamma(r+1)}, \quad r=1,2, \ldots
$$

Also, it is clear that

$$
I_{\nu}(z) \sim \frac{z^{\nu}}{2^{\nu} \Gamma(\nu+1)}, \text { as } z \rightarrow 0^{+},
$$

Moreover, according to [17, (5.7.9)] we have that,

$$
\frac{d}{d z}\left(z^{-\nu} I_{\nu}(z)\right)=z^{-\nu} I_{\nu+1}(z), \quad z \in(0, \infty)
$$

and

$$
I_{\nu+1}(z)=I_{\nu-1}(z)-\frac{2 \nu}{z} I_{\nu}(z), \quad z \in(0, \infty) .
$$

Let now $\alpha=\left(\alpha_{1}, \ldots, \alpha_{m}\right) \in[1 / 2, \infty)^{m}$. The Laguerre operator $-\mathfrak{L}_{\alpha}$ generates the semigroup of contractions $\left\{\mathcal{W}_{t}^{\alpha}\right\}_{t>0}$ in $L^{2}\left((0, \infty)^{m}\right)$, where, for every $t>0$ and $g \in L^{2}\left((0, \infty)^{m}\right)$,

$$
\mathcal{W}_{t}^{\alpha}(g)(x)=\int_{(0, \infty)^{m}} \mathcal{W}_{t}^{\alpha}(x, y) g(y) d y, \quad x \in(0, \infty)^{m}
$$

being

$$
\mathcal{W}_{t}^{\alpha}(x, y)=\prod_{j=1}^{m} W_{t}^{\alpha_{j}}\left(x_{j}, y_{j}\right), \quad t>0, \quad\left(x_{1}, \ldots, x_{m}\right) \in(0, \infty)^{m} \text { and } y=\left(y_{1}, \ldots, y_{m}\right) \in(0, \infty)^{m} .
$$

The family $\left\{\mathcal{W}_{t}^{\alpha}\right\}_{t>0}$ is a semigroup of contractions in $L^{p}\left((0, \infty)^{m}\right), 1 \leq p \leq \infty$. 
Assume that $a>0$. Straightforward manipulations allow us to show that the operator $-\mathfrak{L}_{\alpha}(a)$ generates on $L^{p}(0, \infty), 1 \leq p<\infty$, the semigroup of operators $\left\{\mathcal{W}_{t, a}^{\alpha}\right\}_{t>0}$, where, for every $t>0$,

$$
\mathcal{W}_{t, a}^{\alpha}(g)(x)=\int_{(0, \infty)^{m}} \mathcal{W}_{t}^{\alpha}(x, y ; a) g(y) d y, \quad g \in L^{p}\left((0, \infty)^{m}\right), 1 \leq p<\infty,
$$

being

$$
\mathcal{W}_{t}^{\alpha}(x, y ; a)=a^{m / 2} \mathcal{W}_{t a}^{\alpha}(\sqrt{a} x, \sqrt{a} y), \quad x, y \in(0, \infty)^{m} \text { and } t>0 .
$$

We can write, for every $g \in L^{p}\left((0, \infty)^{m}\right), 1<p<\infty$,

$$
\mathfrak{L}_{\alpha}(a)^{-1 / 2} g(x)=\frac{1}{\sqrt{\pi}} \int_{0}^{\infty} \mathcal{W}_{t, a}^{\alpha}(g)(x) \frac{d t}{\sqrt{t}}, \quad x \in(0, \infty)^{m} .
$$

By using the arguments given in [2], [11] and [22] we can see that, for every $j=1, \ldots, m$, and $g \in L^{2}\left((0, \infty)^{m}\right)$,

$$
R_{\alpha, j}(a)(g)(x)=\lim _{\varepsilon \rightarrow 0^{+}} \int_{|x-y|>\varepsilon} R_{\alpha, j}(x, y ; a) g(y) d y, \quad \text { a.e. } x \in(0, \infty)^{m},
$$

where

and

$$
R_{\alpha, j}(x, y ; a)=\frac{1}{\sqrt{\pi}} \int_{0}^{\infty} A_{\alpha_{j}}(a) \mathcal{W}_{t}^{\alpha}(x, y ; a) \frac{d t}{\sqrt{t}}, \quad x, y \in(0, \infty)^{m}, x \neq y
$$

$$
\widetilde{R}_{\alpha, j}(a)(g)(x)=\lim _{\varepsilon \rightarrow 0^{+}} \int_{|x-y|>\varepsilon} \widetilde{R}_{\alpha, j}(x, y ; a) g(y) d y, \quad \text { a.e. } x \in(0, \infty)^{m},
$$

where

$$
\widetilde{R}_{\alpha, j}(x, y ; a)=\frac{1}{\sqrt{\pi}} \int_{0}^{\infty} A_{\alpha_{j}}^{*}(a) \mathcal{W}_{t}^{\alpha}(x, y ; a) \frac{d t}{\sqrt{t}}, \quad x, y \in(0, \infty)^{m}, x \neq y .
$$

Moreover, the operators $R_{\alpha, j}(a)$ and $\widetilde{R}_{\alpha, j}(a), j=1, \ldots, m$, can be extended from $L^{2}\left((0, \infty)^{m}\right) \cap$ $L^{p}\left((0, \infty)^{m}\right)$ to $L^{p}\left((0, \infty)^{m}\right)$ as a bounded operator from $L^{p}\left((0, \infty)^{m}\right)$ into itself, for every $1<p<$ $\infty$.

We need to recall some definitions related to the Hermite operator which we denote by $\mathcal{H}$.

The operator

can be written as follows

$$
\mathcal{H}=-\Delta+|x|^{2}, \quad \text { on } \mathbb{R}^{m}
$$

$$
\mathcal{H}=-\frac{1}{2} \sum_{j=1}^{m}\left(A_{j} A_{j}^{*}+A_{j}^{*} A_{j}\right)
$$

where

$$
A_{j}=\partial_{x_{j}}+x_{j} \quad \text { and } \quad A_{j}^{*}=-\partial_{x_{j}}+x_{j}, \quad j=1, \ldots, m .
$$

Note that $A_{j}^{*}$ is the "formal" adjoint of $A_{j}$ in $L^{2}\left(\mathbb{R}^{n}\right), j=1, \ldots, m$. For every $k \in \mathbb{N}$, the $k$-th Hermite function $h_{k}$ is defined by

$$
h_{k}(x)=\left(\sqrt{\pi} 2^{k} k !\right)^{-1 / 2} e^{-x^{2} / 2} H_{k}(x), \quad x \in \mathbb{R},
$$

where $H_{k}$ represents the $k$-th Hermite polynomial [17, (4.9.1) and (4.9.2)]. We have that

$$
H h_{k}=(2 k+1) h_{k}, \quad k \in \mathbb{N} .
$$

where $H=-\frac{d^{2}}{d x^{2}}+x^{2}, \quad x \in \mathbb{R}$. The system $\left\{h_{k}\right\}_{k \in \mathbb{N}}$ is an orthonormal basis in $L^{2}(\mathbb{R})$. The operator $-H$ generates the semigroup of contractions $\left\{W_{t}\right\}_{t>0}$ in $L^{2}(\mathbb{R})$, being for every $t>0$,

$$
W_{t}(g)=\sum_{k=0}^{\infty} e^{-t(2 k+1)} b_{k}(g) h_{k}, \quad g \in L^{2}(\mathbb{R})
$$

and

$$
b_{k}(g)=\int_{\mathbb{R}} h_{k}(y) g(y) d y, \quad k \in \mathbb{N} .
$$

By using Mehler's formula for Hermite functions [32, (1.1.36)] we obtain

$$
W_{t}(g)(x)=\int_{\mathbb{R}} W_{t}(x, y) g(y) d y, \quad g \in L^{2}(\mathbb{R}) \text { and } t>0,
$$


where, for $x, y \in \mathbb{R}$ and $t \in(0, \infty)$,

$$
W_{t}(x, y)=\frac{1}{\sqrt{\pi}}\left(\frac{e^{-2 t}}{1-e^{-4 t}}\right)^{1 / 2} \exp \left(-\frac{1}{4}\left[(x-y)^{2} \frac{1+e^{-2 t}}{1-e^{-2 t}}+(x+y)^{2} \frac{1-e^{-2 t}}{1+e^{-2 t}}\right]\right) .
$$

Moreover, if $W_{t}, t>0$, is defined by (25), $\left\{W_{t}\right\}_{t>0}$ is a semigroup of contractions in $L^{p}(\mathbb{R})$, $1 \leq p<\infty$.

The Hermite operator $-\mathcal{H}$ generates, for every $1 \leq p<\infty$, the semigroup of contractions $\left\{\mathcal{W}_{t}\right\}_{t>0}$ in $L^{p}\left(\mathbb{R}^{m}\right)$, where, for every $f \in L^{p}\left(\mathbb{R}^{m}\right)$,

$$
\mathcal{W}_{t}(f)(x)=\int_{\mathbb{R}^{m}} \mathcal{W}_{t}(x, y) f(y) d y, \quad x \in \mathbb{R}^{m},
$$

being

$$
\mathcal{W}_{t}(x, y)=\prod_{j=1}^{m} W_{t}\left(x_{j}, y_{j}\right), \quad x, y \in \mathbb{R}^{m} \text { and } t>0 .
$$

In order to study Riesz transforms associated with Grushin operator Jotsaroop, Sanjay and Thangavelu [16] considered the scaled Hermite operator $\mathcal{H}(a)$ defined by

$$
\mathcal{H}(a)=-\Delta+a^{2}|x|^{2}, \quad \text { on } \mathbb{R}^{m},
$$

for every $a \in \mathbb{R}$. The operator $\mathcal{H}(a)$ can be written as

$$
\mathcal{H}(a)=\frac{1}{2} \sum_{j=1}^{m}\left[A_{j}(a) A_{j}^{*}(a)+A_{j}^{*}(a) A_{j}(a)\right]
$$

where $A_{j}(a)=\frac{d}{d x_{j}}+|a| x_{j}$ and $A_{j}^{*}(a)=-\frac{d}{d x_{j}}+|a| x_{j}, j=1, \ldots, m$. Riesz transforms for the operator $\mathcal{H}(a)$ were formally defined by

$$
R_{j}(a)=A_{j}(a) \mathcal{H}(a)^{-1 / 2} \text { and } \widetilde{R}_{j}(a)=A_{j}^{*}(a) \mathcal{H}(a)^{-1 / 2}, \quad a \in \mathbb{R} \backslash\{0\}, \text { and } j=1, \ldots, m .
$$

Here, we only consider the Riesz transform $R_{1}(a)$. By taking in mind [30, (3.1)] the operator $R_{1}(a)$ is defined in $L^{2}\left(\mathbb{R}^{m}\right)$ as follows

$$
R_{1}(a)(g)=\sum_{k \in \mathbb{N}^{m}} \sqrt{\frac{2 k_{1}}{2 \mathfrak{s}(k)+1}} b_{k}(a)(g) \mathfrak{h}_{k-1}(\cdot ; a), \quad g \in L^{2}\left(\mathbb{R}^{m}\right),
$$

where, for every $k \in \mathbb{N}^{m}$,

$$
b_{k}(a)(g)=\int_{\mathbb{R}^{m}} \mathfrak{h}_{k}(y ; a) g(y) d y,
$$

and $\mathfrak{h}_{k}(x ; a)=|a|^{m / 4} \mathfrak{h}_{k}(\sqrt{|a|} x), x \in \mathbb{R}^{m}$, being $\mathfrak{h}_{k}(x)=\prod_{j=1}^{m} h_{k_{j}}\left(x_{j}\right), x \in \mathbb{R}^{m}$.

$R_{1}(a)$ is a bounded operator in $L^{2}\left(\mathbb{R}^{m}\right)$. Moreover, for every $1<p<\infty, R_{1}(a)$ can be extended from $L^{2}\left(\mathbb{R}^{m}\right) \cap L^{p}\left(\mathbb{R}^{m}\right)$ to $L^{p}\left(\mathbb{R}^{m}\right)$ as a bounded operator from $L^{p}\left(\mathbb{R}^{m}\right)$ into itself, and, for every $g \in L^{p}\left(\mathbb{R}^{m}\right)$,

$$
R_{1}(a)(g)(x)=\lim _{\varepsilon \rightarrow 0^{+}} \int_{|x-y|>\varepsilon} R_{1}(x, y ; a) g(y) d y, \quad \text { a.e. } x \in \mathbb{R}^{m},
$$

where

$$
R_{1}(x, y ; a)=\frac{1}{\sqrt{\pi}} \int_{0}^{\infty}|a|^{m / 2} A_{1}(a) \mathcal{W}_{t|a|}(\sqrt{|a|} x, \sqrt{|a|} y) \frac{d t}{\sqrt{t}}, \quad x, y \in \mathbb{R}^{m}, \quad x \neq y .
$$

Note that $R_{1}(x, y ; a)=|a|^{m / 2} R_{1}(\sqrt{|a|} x, \sqrt{|a|} y ; 1), x, y \in \mathbb{R}^{m}$.

Next, we collect some estimates concerning Hermite and Laguerre heat kernels. Let $\beta \geq 1 / 2$ and $u, v \in(0, \infty)$. It is convenient to write $W_{t}$ and $W_{t}^{\beta}$ in terms of the functions $\mathfrak{a}(t)$ and $\mathfrak{b}(t)$ defined in (17), as follows

$$
W_{t}(u, v)=\frac{\sqrt{\mathfrak{a}(t)}}{\sqrt{2 \pi}} e^{-\mathfrak{b}(t)\left(u^{2}+v^{2}\right)} e^{\mathfrak{a}(t) u v}
$$


and

$$
\begin{aligned}
W_{t}^{\beta}(u, v) & =\sqrt{\mathfrak{a}(t)} \sqrt{\mathfrak{a}(t) u v} I_{\beta}(\mathfrak{a}(t) u v) e^{-\mathfrak{b}(t)\left(u^{2}+v^{2}\right)} \\
& =W_{t}(u, v) \sqrt{2 \pi \mathfrak{a}(t) u v} I_{\beta}(\mathfrak{a}(t) u v) e^{-\mathfrak{a}(t) u v} .
\end{aligned}
$$

Since the asymptotics for the modified Bessel function $I_{\beta}$ depend on whether its argument is small or large (see $\sqrt{19}$ and $(20)$ ) it will be useful to consider the next to sets

$$
A_{1}(u, v)=\{t \in(0, \infty): \mathfrak{a}(t) u v \leq 1\} \quad \text { and } \quad B_{1}(u, v)=\{t \in(0, \infty): \mathfrak{a}(t) u v \geq 1\} .
$$

Lemma 3.1. Let $\beta \geq 1 / 2,0<\varepsilon<1$ and $t, u, v \in(0, \infty)$. Then,

(a) $W_{t}^{\beta}(u, v) \leq C W_{t}(u, v) \leq C \frac{e^{-c|u-v|^{2} / t}}{\sqrt{t}}$.

(b) $\left|W_{t}^{\beta}(u, v)-W_{t}(u, v)\right| \leq C e^{-(1-\varepsilon) t} \begin{cases}\frac{e^{-c|u-v|^{2} / t}}{\sqrt{t}}, & t \in A_{1}(u, v), \\ \frac{1}{\mathfrak{a}(t) u v} \frac{e^{-c|u-v|^{2} / t}}{\sqrt{t}}, & t \in B_{1}(u, v) .\end{cases}$

(c) $\left|\partial_{u}^{\ell} W_{t}(u, v)\right| \leq C \frac{e^{-c|u-v|^{2} / t}}{t^{(\ell+1) / 2}}, \quad \ell=1,2$.

(d) $\left|u \partial_{u} W_{t}(u, v)+v \partial_{v} W_{t}(u, v)\right| \leq C \frac{e^{-c|u-v|^{2} / t}}{\sqrt{t}}$.

(e) $\left|u \partial_{u} W_{t}^{\beta}(u, v)+v \partial_{v} W_{t}^{\beta}(u, v)\right| \leq C\left(1+e^{(1+\varepsilon) t} \chi_{\{u / 2<v<2 u\}}(v)\right) \frac{e^{-c|u-v|^{2} / t}}{\sqrt{t}}$.

$(f)\left|u \partial_{u}\left[W_{t}^{\beta}-W_{t}\right](u, v)+v \partial_{v}\left[W_{t}^{\beta}-W_{t}\right](u, v)\right| \leq C \frac{e^{-(1-\varepsilon) t}}{(\mathfrak{a}(t) u v)^{1 / 4}} \frac{e^{-c|u-v|^{2} / t}}{\sqrt{t}}$.

Proof of $(a)$. The first inequality is a consequence of 27 together with 19 and 20 . The second one follows easily from 26 and $(18)$.

Proof of (b). It is straightforward from the relation (27), Lemma 3.1, (a); and (19).

Proof of $(c)$. It is enough to note that,

$$
\begin{aligned}
\left|\partial_{u} W_{t}(u, v)\right|= & \frac{\sqrt{\mathfrak{a}(t)}}{2 \sqrt{\pi}} \exp \left(-\frac{1}{4}\left[(u-v)^{2} \frac{1+e^{-2 t}}{1-e^{-2 t}}+(u+v)^{2} \frac{1-e^{-2 t}}{1+e^{-2 t}}\right]\right) \\
& \times\left|(u-v) \frac{1+e^{-2 t}}{1-e^{-2 t}}+(u+v) \frac{1-e^{-2 t}}{1+e^{-2 t}}\right| \\
\leq & C \frac{e^{-c|u-v|^{2} / t}}{t} .
\end{aligned}
$$

We can proceed similarly when we take two derivatives.

Proof of $(d)$. Observe that

$$
\begin{aligned}
\left|u \partial_{u} W_{t}(u, v)+v \partial_{v} W_{t}(u, v)\right| \leq & C \sqrt{\mathfrak{a}(t)} \exp \left(-\frac{1}{4}\left[(u-v)^{2} \frac{1+e^{-2 t}}{1-e^{-2 t}}+(u+v)^{2} \frac{1-e^{-2 t}}{1+e^{-2 t}}\right]\right) \\
& \times\left((u-v)^{2} \frac{1+e^{-2 t}}{1-e^{-2 t}}+(u+v)^{2} \frac{1-e^{-2 t}}{1+e^{-2 t}}\right) \\
\leq & C \frac{e^{-c|u-v|^{2} / t}}{\sqrt{t}} .
\end{aligned}
$$


Proof of (e). From 21] we deduce that,

$$
\begin{aligned}
\partial_{u} W_{t}^{\beta}(u, v)= & \sqrt{\mathfrak{a}(t)} \partial_{u}\left[(\mathfrak{a}(t) u v)^{\beta+1 / 2} e^{-\mathfrak{b}(t)\left(u^{2}+v^{2}\right)}(\mathfrak{a}(t) u v)^{-\beta} I_{\beta}(\mathfrak{a}(t) u v)\right] \\
= & \sqrt{\mathfrak{a}(t)}\left[(\beta+1 / 2) \mathfrak{a}(t) v(\mathfrak{a}(t) u v)^{-1 / 2} I_{\beta}(\mathfrak{a}(t) u v)-\sqrt{\mathfrak{a}(t) u v} I_{\beta}(\mathfrak{a}(t) u v) 2 u \mathfrak{b}(t)\right. \\
& \left.+\mathfrak{a}(t) v \sqrt{\mathfrak{a}(t) u v} I_{\beta+1}(\mathfrak{a}(t) u v)\right] e^{-\mathfrak{b}(t)\left(u^{2}+v^{2}\right)}
\end{aligned}
$$

Hence

$$
\begin{aligned}
u \partial_{u} W_{t}^{\beta}(u, v)+v \partial_{v} W_{t}^{\beta}(u, v)= & \sqrt{\mathfrak{a}(t)}\left[\sqrt{\mathfrak{a}(t) u v} I_{\beta}(\mathfrak{a}(t) u v)\left((2 \beta+1)-2 \mathfrak{b}(t)\left(u^{2}+v^{2}\right)\right)\right. \\
& \left.+2(\mathfrak{a}(t) u v)^{3 / 2} I_{\beta+1}(\mathfrak{a}(t) u v)\right] e^{-\mathfrak{b}(t)\left(u^{2}+v^{2}\right)} \\
= & \sqrt{2 \pi} W_{t}(u, v)\left[\sqrt{\mathfrak{a}(t) u v} I_{\beta}(\mathfrak{a}(t) u v)\left((2 \beta+1)-2 \mathfrak{b}(t)\left(u^{2}+v^{2}\right)\right)\right. \\
& \left.+2(\mathfrak{a}(t) u v)^{3 / 2} I_{\beta+1}(\mathfrak{a}(t) u v)\right] e^{-\mathfrak{a}(t) u v}
\end{aligned}
$$

By (19), 20) and 26] we obtain

$$
\begin{aligned}
\left|u \partial_{u} W_{t}^{\beta}(u, v)+v \partial_{v} W_{t}^{\beta}(u, v)\right| & \leq C\left(1+\mathfrak{b}(t)\left(u^{2}+v^{2}\right)+\mathfrak{a}(t) u v\right) \frac{e^{-c|u-v|^{2} /\left(1-e^{-2 t}\right)}}{\sqrt{t}} \\
& \leq C \frac{e^{-c|u-v|^{2} / t}}{\sqrt{t}}, \quad v \in(0, u / 2] \cup[2 u, \infty) .
\end{aligned}
$$

Suppose now that $0<u / 2<v<2 u$. Applying 20 and Lemma 3.1. (a); we get for each $t \in A_{1}(u, v)$,

$$
\begin{aligned}
\left|u \partial_{u} W_{t}^{\beta}(u, v)+v \partial_{v} W_{t}^{\beta}(u, v)\right| & \leq C\left(1+\mathfrak{b}(t) u^{2}\right) e^{-c \mathfrak{a}(t) u^{2}} W_{t}(u, v) \\
& \leq C\left(1+e^{2 t}\right) W_{t}(u, v) \leq C\left(1+e^{(1+\varepsilon) t}\right) \frac{e^{-c|u-v|^{2} / t}}{\sqrt{t}} .
\end{aligned}
$$

On the other hand, 19 with $n=0$ give us, for $t \in B_{1}(u, v)$,

$$
\begin{gathered}
\left|-2 \mathfrak{b}(t)\left(u^{2}+v^{2}\right) \sqrt{\mathfrak{a}(t) u v} I_{\beta}(\mathfrak{a}(t) u v)+2(\mathfrak{a}(t) u v)^{3 / 2} I_{\beta+1}(\mathfrak{a}(t) u v)\right| e^{-\mathfrak{a}(t) u v} \\
\leq C\left(\left|-2 \mathfrak{b}(t)\left(u^{2}+v^{2}\right)+2 \mathfrak{a}(t) u v\right|+\frac{2 \mathfrak{b}(t)\left(u^{2}+v^{2}\right)+2 \mathfrak{a}(t) u v}{\mathfrak{a}(t) u v}\right) \\
\leq C\left((u+v)^{2} \frac{1-e^{-2 t}}{1+e^{-2 t}}+(u-v)^{2} \frac{1+e^{-2 t}}{1-e^{-2 t}}+\frac{\left(u^{2}+v^{2}\right) e^{2 t}+u v}{u v}\right) .
\end{gathered}
$$

Thus, for every $t \in B_{1}(u, v)$,

$$
\left|u \partial_{u} W_{t}^{\beta}(u, v)+v \partial_{v} W_{t}^{\beta}(u, v)\right| \leq C\left(1+e^{(1+\varepsilon) t}\right) \frac{e^{-c|u-v|^{2} / t}}{\sqrt{t}} .
$$


Proof of $(f)$. According to 29 we get,

$$
\begin{aligned}
u \partial_{u}\left[W_{t}^{\beta}-\right. & \left.W_{t}\right](u, v)+v \partial_{v}\left[W_{t}^{\beta}-W_{t}\right](u, v) \\
= & u \partial_{u}\left[W_{t}(u, v)\left(\sqrt{2 \pi \mathfrak{a}(t) u v} I_{\beta}(\mathfrak{a}(t) u v) e^{-\mathfrak{a}(t) u v}-1\right)\right] \\
& \quad+v \partial_{v}\left[W_{t}(u, v)\left(\sqrt{2 \pi \mathfrak{a}(t) u v} I_{\beta}(\mathfrak{a}(t) u v) e^{-\mathfrak{a}(t) u v}-1\right)\right] \\
= & {\left[u \partial_{u} W_{t}(u, v)+v \partial_{v} W_{t}(u, v)\right]\left(\sqrt{2 \pi \mathfrak{a}(t) u v} I_{\beta}(\mathfrak{a}(t) u v) e^{-\mathfrak{a}(t) u v}-1\right) } \\
& \quad+W_{t}(u, v)\left(u \partial_{u}+v \partial_{v}\right)\left(\sqrt{2 \pi \mathfrak{a}(t) u v} I_{\beta}(\mathfrak{a}(t) u v) e^{-\mathfrak{a}(t) u v}\right) \\
= & \frac{\sqrt{\mathfrak{a}(t)}}{2 \sqrt{\pi}}\left[(u-v)^{2} \frac{1+e^{-2 t}}{1-e^{-2 t}}+(u+v)^{2} \frac{1-e^{-2 t}}{1+e^{-2 t}}\right] \\
\quad & \quad \exp \left(-\frac{1}{4}\left[(u-v)^{2} \frac{1+e^{-2 t}}{1-e^{-2 t}}+(u+v)^{2} \frac{1-e^{-2 t}}{1+e^{-2 t}}\right]\right)\left(\sqrt{2 \pi \mathfrak{a}(t) a u v} I_{\beta}(\mathfrak{a}(t) a u v) e^{-\mathfrak{a}(t) a u v}-1\right) \\
& \quad+W_{t}(u, v)\left(u \partial_{u}+v \partial_{v}\right)\left(\sqrt{2 \pi \mathfrak{a}(t) u v} I_{\beta}(\mathfrak{a}(t) u v) e^{-\mathfrak{a}(t) u v}\right) .
\end{aligned}
$$

By 21) we have that

$$
\begin{aligned}
\frac{d}{d z}\left[\sqrt{2 \pi z} I_{\beta}(z) e^{-z}\right] & =\sqrt{2 \pi} \frac{d}{d z}\left[z^{\beta+1 / 2} z^{-\beta} I_{\beta}(z) e^{-z}\right] \\
& =\frac{\beta+1 / 2}{z} \sqrt{2 \pi z} I_{\beta}(z) e^{-z}+\sqrt{2 \pi z} I_{\beta+1}(z) e^{-z}-\sqrt{2 \pi z} I_{\beta}(z) e^{-z}, \quad z \in(0, \infty)
\end{aligned}
$$

Then, by using 20 we obtain,

$$
\left|\frac{d}{d z}\left(\sqrt{2 \pi z} I_{\beta}(z) e^{-z}\right)\right| \leq C z^{\beta-1 / 2}, \quad z \in(0,1) .
$$

From (19) and (31) we deduce that, for every $n=2,3, \ldots$,

$$
\begin{aligned}
\frac{d}{d z}( & \left.\sqrt{2 \pi z} I_{\beta}(z) e^{-z}\right)=\sqrt{2 \pi}\left(\frac{\beta+1 / 2}{z} z^{1 / 2} I_{\beta}(z) e^{-z}+z^{1 / 2} I_{\beta+1}(z) e^{-z}-z^{1 / 2} I_{\beta}(z) e^{-z}\right) \\
= & \frac{\beta+1 / 2}{z}\left(\sum_{r=0}^{n}(-1)^{r} \frac{[\beta, r]}{(2 z)^{r}}+\mathcal{O}\left(\frac{1}{z^{n+1}}\right)\right)+\sum_{r=0}^{n}(-1)^{r} \frac{[\beta+1, r]}{(2 z)^{r}}+\mathcal{O}\left(\frac{1}{z^{n+1}}\right) \\
& -\sum_{r=0}^{n}(-1)^{r} \frac{[\beta, r]}{(2 z)^{r}}+\mathcal{O}\left(\frac{1}{z^{n+1}}\right) \\
= & \sum_{r=1}^{n}(-1)^{r} \frac{-(2 \beta+1)[\beta, r-1]+[\beta+1, r]-[\beta, r]}{(2 z)^{r}}+\mathcal{O}\left(\frac{1}{z^{n+1}}\right), \quad z \in(0, \infty) .
\end{aligned}
$$

Since,

$$
-(2 \beta+1)[\beta, r-1]+[\beta+1, r]-[\beta, r]=2(r-1)[\beta, r-1], \quad r=1,2,3, \ldots,
$$

we get

$$
\frac{d}{d z}\left(\sqrt{2 \pi z} I_{\beta}(z) e^{-z}\right)=\sum_{r=2}^{n}(-1)^{r} \frac{2(r-1)[\beta, r-1]}{(2 z)^{r}}+\mathcal{O}\left(\frac{1}{z^{n+1}}\right), \quad z \in(0, \infty),
$$

for every $n=2,3, \ldots$ Then,

$$
\frac{d}{d z}\left(\sqrt{2 \pi z} I_{\beta}(z) e^{-z}\right)=\mathcal{O}\left(\frac{1}{z^{2}}\right), \quad z \in(0, \infty) .
$$

According to 19 and 20 it follows that

$$
\begin{aligned}
\mid \sqrt{\mathfrak{a}(t)}[ & \left.(u-v)^{2} \frac{1+e^{-2 t}}{1-e^{-2 t}}+(u+v)^{2} \frac{1-e^{-2 t}}{1+e^{-2 t}}\right] \exp \left(-\frac{1}{4}\left[(u-v)^{2} \frac{1+e^{-2 t}}{1-e^{-2 t}}+(u+v)^{2} \frac{1-e^{-2 t}}{1+e^{-2 t}}\right]\right) \\
& \times\left(\sqrt{2 \pi \mathfrak{a}(t) u v} I_{\beta}(\mathfrak{a}(t) u v) e^{-\mathfrak{a}(t) u v}-1\right) \mid \\
\leq & C \frac{e^{-(1-\varepsilon) t}}{(\mathfrak{a}(t) u v)^{1 / 4}} \frac{e^{-c|u-v|^{2} / t}}{\sqrt{t}}, \quad u, v, t \in(0, \infty) .
\end{aligned}
$$


Moreover, 32 and 33 imply that

$$
\left|W_{t}(u, v)\left(u \partial_{u}+v \partial_{v}\right)\left(\sqrt{2 \pi \mathfrak{a}(t) u v} I_{\beta}(\mathfrak{a}(t) u v) e^{-\mathfrak{a}(t) u v}\right)\right| \leq C \frac{e^{-(1-\varepsilon) t}}{(\mathfrak{a}(t) u v)^{1 / 4}} \frac{e^{-c|u-v|^{2} / t}}{\sqrt{t}} .
$$

Recall that, for every $t, u, v \in(0, \infty)$,

$$
G_{t}^{\beta}(u, v)=A_{\beta}(1) W_{t}^{\beta}(u, v) \quad \text { and } \quad G_{t}(u, v)=A(1) W_{t}(u, v),
$$

being $A_{\beta}(1)$ and $A(1)$ the usual derivatives in the Laguerre and Hermite setting, respectively (see (1) and (24)).

Lemma 3.2. Let $\beta \geq 1 / 2,0<\varepsilon<1$ and $t, u, v \in(0, \infty)$. Then,

(a) $\left|G_{t}(u, v)\right| \leq C e^{-(3-\varepsilon) t} \frac{e^{-c|u-v|^{2} / t}}{t}$.

(b) $\left|G_{t}^{\beta}(u, v)-G_{t}(u, v)\right| \leq C \begin{cases}\frac{e^{-(3 / 2-\varepsilon) t}}{\sqrt{u}} \frac{e^{-c|u-v|^{2} / t}}{t^{3 / 4}}, & u / 2<v<2 u, \\ e^{-(3-\varepsilon) t} \max \{u, v\} \frac{e^{-c|u-v|^{2} / t}}{t^{3 / 2}}, & v \in(0, u / 2] \cup[2 u, \infty) .\end{cases}$

(c) $\left|u \partial_{u} G_{t}(u, v)+v \partial_{v} G_{t}(u, v)\right| \leq C \frac{e^{-c|u-v|^{2} / t}}{t}$.

(d) $\left|\partial_{u}\left[G_{t}^{\beta}-G_{t}\right](u, v)\right| \leq C \frac{e^{-c\left(u^{2}+v^{2}\right) / t}}{t^{3 / 2}}, \quad t \in A_{1}(u, v)$.

(e) $\left|u \partial_{u}\left[G_{t}^{\beta}-G_{t}\right](u, v)+v \partial_{v}\left[G_{t}^{\beta}-G_{t}\right](u, v)\right| \leq C\left(\frac{u^{1 / 4}}{v^{3 / 4}}+\frac{v^{1 / 4}}{u^{3 / 4}}\right) \frac{e^{-c|u-v|^{2} / t}}{t^{3 / 4}}, \quad t \in B_{1}(u, v)$.

$(f)\left|u \partial_{u}\left[G_{t}^{\beta}-G_{t}\right](u, v)+v \partial_{v}\left[G_{t}^{\beta}-G_{t}\right](u, v)\right| \leq C \frac{e^{-c \max \{u, v\}^{2} / t}}{t}, \quad v \in(0, u / 2] \cup[2 u, \infty)$.

(g) $\left|u \partial_{u}^{2}\left[G_{t}^{\beta}-G_{t}\right](u, v)\right| \leq C \begin{cases}\frac{e^{-c\left(u^{2}+v^{2}\right) / t}}{t^{3 / 2}}, & t \in A_{1}(u, v), \\ \frac{e^{-c|u-v|^{2} / t}}{t^{3 / 2}}, & t \in B_{1}(u, v) .\end{cases}$

Proof of (a). We have that

$$
G_{t}(u, v)=(\mathfrak{a}(t) v+(1-2 \mathfrak{b}(t)) u) W_{t}(u, v) .
$$

Thus,

$\left|G_{t}(u, v)\right| \leq C e^{-3 t} \frac{|u-v|+u\left(1-e^{-2 t}\right)}{1-e^{-4 t}} \frac{e^{-c\left(|u-v|^{2} /\left(1-e^{-2 t}\right)+|u+v|^{2}\left(1-e^{-2 t}\right)\right)}}{\sqrt{t}} \leq C e^{-(3-\varepsilon) t} \frac{e^{-c|u-v|^{2} / t}}{t}$.

Proof of (b). By taking into account that

$$
A_{\beta}(1)=\frac{d}{d u}+u-\frac{\beta+1 / 2}{u}=u^{\beta+1 / 2} \frac{d}{d u} u^{-\beta-1 / 2}+u,
$$


(21) leads to

$$
\begin{aligned}
G_{t}^{\beta}(u, v)= & u W_{t}^{\beta}(u, v)+\mathfrak{a}(t)^{\beta+1}(u v)^{\beta+1 / 2} \partial_{u}\left[e^{-\mathfrak{b}(t)\left(u^{2}+v^{2}\right)}(\mathfrak{a}(t) u v)^{-\beta} I_{\beta}(\mathfrak{a}(t) u v)\right] \\
= & \mathfrak{a}(t)^{\beta+1}(u v)^{\beta+1 / 2} e^{-\mathfrak{b}(t)\left(u^{2}+v^{2}\right)} \\
& \times\left[(\mathfrak{a}(t) u v)^{-\beta} I_{\beta}(\mathfrak{a}(t) u v)(1-2 \mathfrak{b}(t)) u+(\mathfrak{a}(t) u v)^{-\beta} I_{\beta+1}(\mathfrak{a}(t) u v) v \mathfrak{a}(t)\right] \\
= & u W_{t}(u, v) \sqrt{2 \pi \mathfrak{a}(t) u v} I_{\beta}(\mathfrak{a}(t) u v) e^{-\mathfrak{a}(t) u v} \\
& +W_{t}(u, v)\left[-2 \mathfrak{b}(t) u \sqrt{2 \pi \mathfrak{a}(t) u v} I_{\beta}(\mathfrak{a}(t) u v) e^{-\mathfrak{a}(t) u v}\right. \\
& \left.+\mathfrak{a}(t) v \sqrt{2 \pi \mathfrak{a}(t) u v} I_{\beta+1}(\mathfrak{a}(t) u v) e^{-\mathfrak{a}(t) u v}\right], \quad t, u, v \in(0, \infty) .
\end{aligned}
$$

Putting together (34) and (35) we arrive at

$$
\begin{aligned}
G_{t}^{\beta}(u, v)-G_{t}(u, v)= & u W_{t}(u, v)\left(\sqrt{2 \pi \mathfrak{a}(t) u v} I_{\beta}(\mathfrak{a}(t) u v) e^{-\mathfrak{a}(t) u v}-1\right) \\
& +W_{t}(u, v)\left[-2 \mathfrak{b}(t) u\left(\sqrt{2 \pi \mathfrak{a}(t) u v} I_{\beta}(\mathfrak{a}(t) u v) e^{-\mathfrak{a}(t) u v}-1\right)\right. \\
& \left.+\mathfrak{a}(t) v\left(\sqrt{2 \pi \mathfrak{a}(t) u v} I_{\beta+1}(\mathfrak{a}(t) u v) e^{-\mathfrak{a}(t) u v}-1\right)\right] .
\end{aligned}
$$

First of all, assume that $u / 2<v<2 u$. From (19) with $n=0$ it follows that,

$$
\begin{aligned}
\left|G_{t}^{\beta}(u, v)-G_{t}(u, v)\right| & \leq C \frac{|1-2 \mathfrak{b}(t)| u+\mathfrak{a}(t) v}{(\mathfrak{a}(t) u v)^{3 / 4}} W_{t}(u, v) \leq C \frac{\mathfrak{a}(t)^{1 / 4}}{\sqrt{u}} W_{t}(u, v) \\
& \leq C \frac{e^{-(3 / 2-\varepsilon) t}}{\sqrt{u}} \frac{e^{-c|u-v|^{2} / t}}{t^{3 / 4}}, \quad t \in B_{1}(u, v) .
\end{aligned}
$$

Also 200 leads to,

$$
\begin{aligned}
\left|G_{t}^{\beta}(u, v)-G_{t}(u, v)\right| & \leq C(|1-2 \mathfrak{b}(t)| u+\mathfrak{a}(t) v) W_{t}(u, v) \leq C \frac{\mathfrak{a}(t) u}{(\mathfrak{a}(t) u v)^{3 / 4}} W_{t}(u, v) \\
& \leq C \frac{e^{-(3 / 2-\varepsilon) t}}{\sqrt{u}} \frac{e^{-c|u-v|^{2} / t}}{t^{3 / 4}}, \quad t \in A_{1}(u, v) .
\end{aligned}
$$

Suppose now that $v \in(0, u / 2] \cup[2 u, \infty)$. By proceeding as in 37 and 38 we can obtain

$$
\left|G_{t}^{\beta}(u, v)-G_{t}(u, v)\right| \leq C \mathfrak{a}(t)(u+v) W_{t}(u, v) \leq C e^{-(3-\varepsilon) t} \max \{u, v\} \frac{e^{-c|u-v|^{2} / t}}{t^{3 / 2}} .
$$

Proof of $(c)$. By (34) we can write

$$
u \partial_{u} G_{t}(u, v)+v \partial_{v} G_{t}(u, v)=G_{t}(u, v)+(\mathfrak{a}(t) v+(1-2 \mathfrak{b}(t)) u)\left(u \partial_{u} W_{t}(u, v)+v \partial_{v} W_{t}(u, v)\right) .
$$

Since,

$$
\mathfrak{a}(t) v+(1-2 \mathfrak{b}(t)) u=\mathfrak{a}(t)\left((v-u)+\left(1-e^{-2 t}\right) u\right),
$$

Lemma 3.2 (a), and the first estimate in 28 allow us to deduce the desired conclusion.

Proof of $(d)$. Identity (36) allows us to write

$$
\begin{aligned}
& \partial_{u}\left[G_{t}^{\beta}-G_{t}\right](u, v)=(1-2 \mathfrak{b}(t))\left[W_{t}(u, v)\left(\sqrt{2 \pi \mathfrak{a}(t) u v} I_{\beta}(\mathfrak{a}(t) u v) e^{-\mathfrak{a}(t) u v}-1\right)\right. \\
&+ u \partial_{u} W_{t}(u, v)\left(\sqrt{2 \pi \mathfrak{a}(t) u v} I_{\beta}(\mathfrak{a}(t) u v) e^{-\mathfrak{a}(t) u v}-1\right) \\
&\left.-u W_{t}(u, v) \partial_{u}\left(\sqrt{2 \pi \mathfrak{a}(t) u v} I_{\beta}(\mathfrak{a}(t) u v) e^{-\mathfrak{a}(t) u v}\right)\right] \\
&+\mathfrak{a}(t) v\left[\partial_{u} W_{t}(u, v)\left(\sqrt{2 \pi \mathfrak{a}(t) u v} I_{\beta+1}(\mathfrak{a}(t) u v) e^{-\mathfrak{a}(t) u v}-1\right)\right. \\
&\left.-W_{t}(u, v) \partial_{u}\left(\sqrt{2 \pi \mathfrak{a}(t) u v} I_{\beta+1}(\mathfrak{a}(t) u v) e^{-\mathfrak{a}(t) u v}\right)\right] .
\end{aligned}
$$


By taking into account 20 and 32 we conclude

$$
\begin{aligned}
\left|\partial_{u}\left[G_{t}^{\beta}-G_{t}\right](u, v)\right| & \leq C \mathfrak{a}(t)\left((1+v+u) W_{t}(u, v)+(u+v)\left|\partial_{u} W_{t}(u, v)\right|\right) \\
& \leq C \frac{e^{-c\left(u^{2}+v^{2}\right) / t}}{t^{3 / 2}}, \quad t \in A_{1}(u, v) .
\end{aligned}
$$

Proof of (e). According to (21) and the identity (36) we can write

$$
\begin{gathered}
u \partial_{u}\left(G_{t}^{\beta}(u, v)-G_{t}(u, v)\right)+v \partial_{v}\left(G_{t}(u, v)-G_{t}^{\beta}(u, v)\right) \\
=(1-2 \mathfrak{b}(t))\left[u W_{t}(u, v)\left(\sqrt{2 \pi \mathfrak{a}(t) u v} I_{\beta}(\mathfrak{a}(t) u v) e^{-\mathfrak{a}(t) u v}-1\right)\right. \\
+u^{2} \partial_{u} W_{t}(u, v)\left(\sqrt{2 \pi \mathfrak{a}(t) u v} I_{\beta}(\mathfrak{a}(t) u v) e^{-\mathfrak{a}(t) u v}-1\right) \\
-u^{2} W_{t}(u, v) \partial_{u}\left(\sqrt{2 \pi \mathfrak{a}(t) u v} I_{\beta}(\mathfrak{a}(t) u v) e^{-\mathfrak{a}(t) u v}\right) \\
+u v \partial_{v} W_{t}(u, v)\left(\sqrt{2 \pi \mathfrak{a}(t) u v} I_{\beta} G_{t}^{\beta}-(\mathfrak{a}(t) u v) e^{-\mathfrak{a}(t) u v}-1\right) \\
\left.-u v W_{t}(u, v) \partial_{v}\left(\sqrt{2 \pi \mathfrak{a}(t) u v} I_{\beta}(\mathfrak{a}(t) u v) e^{-\mathfrak{a}(t) u v}\right)\right] \\
+\mathfrak{a}(t)\left[u v \partial_{u} W_{t}(u, v)\left(\sqrt{2 \pi \mathfrak{a}(t) u v} I_{\beta+1}(\mathfrak{a}(t) u v) e^{-\mathfrak{a}(t) u v}-1\right)\right. \\
-u v W_{t}(u, v) \partial_{u}\left(\sqrt{2 \pi \mathfrak{a}(t) u v} I_{\beta+1}(\mathfrak{a}(t) u v) e^{-\mathfrak{a}(t) u v}\right) \\
+v W_{t}(u, v)\left(1-\sqrt{2 \pi \mathfrak{a}(t) u v} I_{\beta+1}(\mathfrak{a}(t) u v) e^{-\mathfrak{a}(t) u v}\right) \\
+v^{2} \partial_{v} W_{t}(u, v)\left(\sqrt{2 \pi \mathfrak{a}(t) u v} I_{\beta+1}(\mathfrak{a}(t) u v) e^{-\mathfrak{a}(t) u v}-1\right) \\
\left.-v^{2} W_{t}(u, v) \partial_{v}\left(\sqrt{2 \pi \mathfrak{a}(t) u v} I_{\beta+1}(\mathfrak{a}(t) u v) e^{-\mathfrak{a}(t) u v}\right)\right] \\
=\sum_{j=1}^{10} H_{j}(t, u, v) .
\end{gathered}
$$

Let $t \in B_{1}(u, v)$. By using (19) we obtain,

$$
\left|H_{1}(t, u, v)+H_{8}(t, u, v)\right| \leq C \frac{|1-2 \mathfrak{b}(t)|}{(\mathfrak{a}(t) u v)^{3 / 4}}(u+v) W_{t}(u, v) \leq C\left(\frac{u^{1 / 4}}{v^{3 / 4}}+\frac{v^{1 / 4}}{u^{3 / 4}}\right) \frac{e^{-c|u-v|^{2} / t}}{t^{3 / 4}} .
$$

Asymptotic behavior 19 leads also to

$$
\begin{aligned}
\left|H_{2}(t, u, v)+H_{4}(t, u, v)\right| & \leq C \frac{\mathfrak{a}(t) u}{(\mathfrak{a}(t) u v)^{3 / 4}}\left|u \partial_{u} W_{t}(u, v)+v \partial_{v} W_{t}(u, v)\right| \\
& =C \frac{\mathfrak{a}(t)^{3 / 4} u^{1 / 4}}{v^{3 / 4}}\left[\mathfrak{b}(t)\left(u^{2}+v^{2}\right)-\mathfrak{a}(t) u v\right] e^{-\mathfrak{b}(t)\left(u^{2}+v^{2}\right)+\mathfrak{a}(t) u v} \\
& \leq C \frac{u^{1 / 4}}{v^{3 / 4}} \frac{e^{-c|u-v|^{2} / t}}{t^{3 / 4}}
\end{aligned}
$$

Also, we have that

$$
\left|H_{6}(t, u, v)+H_{9}(t, u, v)\right| \leq C \frac{\mathfrak{a}(t) v}{(\mathfrak{a}(t) u v)^{3 / 4}}\left|u \partial_{u} W_{t}(u, v)+v \partial_{v} W_{t}(u, v)\right| \leq C \frac{v^{1 / 4}}{u^{3 / 4}} \frac{e^{-c|u-v|^{2} / t}}{t^{3 / 4}} .
$$

According to 33 we get,

$$
\left|H_{3}(t, u, v)+H_{5}(t, u, v)\right| \leq C \frac{\mathfrak{a}(t)^{2} u^{2} v}{(\mathfrak{a}(t) u v)^{7 / 4}} W_{t}(u, v) \leq C \frac{u^{1 / 4}}{v^{3 / 4}} \frac{e^{-c|u-v|^{2} / t}}{t^{3 / 4}} .
$$

Similarly we can show that

$$
\left|H_{7}(t, \sqrt{a} u, \sqrt{a} v)+H_{10}(\sqrt{a} u, \sqrt{a} v, t)\right| \leq C \frac{v^{1 / 4}}{u^{3 / 4}} \frac{e^{-c|u-v|^{2} / t}}{t^{3 / 4}} .
$$


Proof of $(f)$. According to (30) and by using (19), 20, , 32) and (33) we obtain

$$
\begin{aligned}
& \left|u \partial_{u}\left(G_{t}(u, v)-G_{t}^{\beta}(u, v)\right)+v \partial_{v}\left(G_{t}(u, v)-G_{t}^{\beta}(u, v)\right)\right| \\
& \quad \leq C \mathfrak{a}(t)\left\{(u+v) W_{t}(u, v)+u^{2}\left|\partial_{u} W_{t}(u, v)\right|+v^{2}\left|\partial_{v} W_{t}(u, v)\right|+u v\left(\left|\partial_{u} W_{t}(u, v)\right|+\left|\partial_{v} W_{t}(u, v)\right|\right)\right\} \\
& \quad \leq \frac{C}{t^{3 / 2}}\left[u+v+\frac{u^{2}+v^{2}}{\sqrt{t}}\right] e^{-c|u-v|^{2} / t} \leq \frac{C}{t}\left\{\begin{array}{l}
e^{-c u^{2} / t}, \quad 0<v<u / 2, \\
e^{-c v^{2} / t}, \quad 0<2 u<v .
\end{array}\right.
\end{aligned}
$$

Proof of $(g)$. We have that

$$
\begin{aligned}
\partial_{u}^{2}\left[G_{t}^{\beta}-G_{t}\right](u, v)=(1- & 2 \mathfrak{b}(t))\left\{2 \partial_{u}\left[W_{t}(u, v)\left(\sqrt{2 \pi \mathfrak{a}(t) u v} I_{\beta}(\mathfrak{a}(t) u v) e^{-\mathfrak{a}(t) u v}-1\right)\right]\right. \\
+ & u\left[\partial_{u}^{2} W_{t}(u, v)\left(\sqrt{2 \pi \mathfrak{a}(t) u v} I_{\beta}(\mathfrak{a}(t) u v) e^{-\mathfrak{a}(t) u v}-1\right)\right. \\
& -2 \partial_{u} W_{t}(u, v) \partial_{u}\left(\sqrt{2 \pi \mathfrak{a}(t) u v} I_{\beta}(\mathfrak{a}(t) u v) e^{-\mathfrak{a}(t) u v}\right) \\
& \left.\left.-W_{t}(u, v) \partial_{u}^{2}\left(\sqrt{2 \pi \mathfrak{a}(t) u v} I_{\beta}(\mathfrak{a}(t) u v) e^{-\mathfrak{a}(t) u v}\right)\right]\right\} \\
+ & \mathfrak{a}(t) v\left\{\partial_{u}^{2} W_{t}(u, v)\left(\sqrt{2 \pi \mathfrak{a}(t) u v} I_{\beta+1}(\mathfrak{a}(t) u v) e^{-\mathfrak{a}(t) u v}-1\right)\right. \\
& -2 \partial_{u} W_{t}(u, v) \partial_{u}\left(\sqrt{2 \pi \mathfrak{a}(t) u v} I_{\beta+1}(\mathfrak{a}(t) u v) e^{-\mathfrak{a}(t) u v}\right) \\
& \left.-W_{t}(u, v) \partial_{u}^{2}\left(\sqrt{2 \pi \mathfrak{a}(t) u v} I_{\beta+1}(\mathfrak{a}(t) u v) e^{-\mathfrak{a}(t) u v}\right)\right\}
\end{aligned}
$$

By using (21) and 22, we get

$$
\frac{d^{2}}{d z^{2}}\left[\sqrt{z} I_{\beta}(z) e^{-z}\right]=e^{-z}\left[\left(\frac{4 \beta^{2}-1}{4 z^{2}}+2-\frac{2 \beta+1}{z}\right) \sqrt{z} I_{\beta}(z)-2 \sqrt{z} I_{\beta+1}(z)\right], \quad z \in(0, \infty) .
$$

According to 19 it follows that

$$
\frac{d^{2}}{d z^{2}}\left[\sqrt{z} I_{\beta}(z) e^{-z}\right]=\mathcal{O}\left(\frac{1}{z^{3}}\right), \quad z \in(0, \infty) .
$$

Equalities (19), 33), 39), 411) and Lemma 3.1. (c), lead to

$$
\begin{aligned}
\left|u \partial_{u}^{2}\left[G_{t}^{\beta}-G_{t}\right](u, v)\right| \leq & C u \mathfrak{a}(t)\left\{\left|\partial_{u} W_{t}(u, v)\right| \frac{1}{(\mathfrak{a}(t) u v)^{1 / 2}}+W_{t}(u, v) \frac{\mathfrak{a}(t) v}{\mathfrak{a}(t) u v}\right. \\
& +u\left|\partial_{u}^{2} W_{t}(u, v)\right| \frac{1}{\mathfrak{a}(t) u v}+u\left|\partial_{u} W_{t}(u, v)\right| \frac{\mathfrak{a}(t) v}{(\mathfrak{a}(t) u v)^{3 / 2}}+u W_{t}(u, v) \frac{(\mathfrak{a}(t) v)^{2}}{(\mathfrak{a}(t) u v)^{2}} \\
& \left.+v\left|\partial_{u}^{2} W_{t}(u, v)\right| \frac{1}{\mathfrak{a}(t) u v}+v\left|\partial_{u} W_{t}(u, v)\right| \frac{\mathfrak{a}(t) v}{(\mathfrak{a}(t) u v)^{3 / 2}}+v W_{t}(u, v) \frac{(\mathfrak{a}(t) v)^{2}}{(\mathfrak{a}(t) u v)^{2}}\right\} \\
\leq & C \frac{e^{-c|u-v|^{2} / t}}{t^{3 / 2}}, \quad t \in B_{1}(u, v), 0<u / 2<v<2 u .
\end{aligned}
$$

Analogously, when $0<v<u / 2$ or $0<2 u<v$, we get

$$
\begin{aligned}
\left|u \partial_{u}^{2}\left[G_{t}^{\beta}-G_{t}\right](u, v)\right| \leq & C u \mathfrak{a}(t)\left\{\left|\partial_{u} W_{t}(u, v)\right|+W_{t}(u, v) \mathfrak{a}(t) v+u\left|\partial_{u}^{2} W_{t}(u, v)\right|\right. \\
& +u\left|\partial_{u} W_{t}(u, v)\right| \mathfrak{a}(t) v+u W_{t}(u, v)(\mathfrak{a}(t) v)^{2}+v\left|\partial_{u}^{2} W_{t}(u, v)\right| \\
& \left.+v\left|\partial_{u} W_{t}(u, v)\right| \mathfrak{a}(t) v+v W_{t}(u, v)(\mathfrak{a}(t) v)^{2}\right\} \\
\leq & C \frac{u}{t}\left\{\frac{1}{t}+\frac{u+v}{t^{3 / 2}}+\frac{u v+v^{2}}{t^{2}}+\frac{u v^{2}+v^{3}}{t^{5 / 2}}\right\} e^{-c|u-v|^{2} / t} \\
\leq & C \frac{e^{-c|u-v|^{2} / t}}{t^{3 / 2}}, \quad t \in B_{1}(u, v) .
\end{aligned}
$$

On the other hand, from 20,32 and 40 we deduce, for every $z \in(0,1]$,

$$
\left|\frac{d^{\ell}}{d z^{\ell}}\left(\sqrt{z} I_{\beta}(z) e^{-z}\right)\right| \leq C, \quad \ell=0,1, \quad\left|\frac{d^{2}}{d z^{2}}\left(\sqrt{z} I_{\beta}(z) e^{-z}\right)\right| \leq \frac{C}{z} .
$$


Hence,

$$
\begin{aligned}
\left|u \partial_{u}^{2}\left[G_{t}^{\beta}-G_{t}\right](u, v)\right| & \leq C \mathfrak{a}(t)\left\{\left(1+\mathfrak{a}(t) v^{2}\right) W_{t}(u, v)+(u+v)\left|\partial_{u} W_{t}(u, v)\right|+\left(u^{2}+v^{2}\right)\left|\partial_{u}^{2} W_{t}(u, v)\right|\right\} \\
& \leq C \frac{e^{-c\left(u^{2}+v^{2}\right) / t}}{t^{3 / 2}}, \quad t \in A_{1}(u, v) .
\end{aligned}
$$

3.2. The proof of the Theorem. In this section we prove that the Riesz transform $R_{\alpha, 1}$ is bounded from $L^{p}\left((0, \infty)^{m} \times \mathbb{R}\right)$ into itself, for every $1<p<\infty$. The boundedness of the other Riesz Transforms can be showed in a similar way.

The operator $R_{\alpha, 1}$ is defined by

$$
R_{\alpha, 1}(f)(x, y)=\mathcal{F}_{2}^{-1}\left(R_{\alpha, 1}(|u|)\left(\mathcal{F}_{2}(f)\right)(x, u)\right)(y), \quad f \in L^{2}\left((0, \infty)^{m} \times \mathbb{R}\right) .
$$

Let $1<p<\infty$. We identify $L^{p}\left((0, \infty)^{m} \times \mathbb{R}\right)$ with $L^{p}\left(\mathbb{R}, L^{p}\left((0, \infty)^{m}\right)\right)$. The Riesz transform $R_{\alpha, 1}$ can be understood as a Banach valued Fourier multiplier. Indeed, let $f \in C_{c}^{\infty}\left((0, \infty)^{m} \times \mathbb{R}\right) \subseteq$ $S\left(\mathbb{R}, L^{p}\left((0, \infty)^{m}\right)\right)$, where $S\left(\mathbb{R}, L^{p}\left((0, \infty)^{m}\right)\right)$ denotes the $L^{p}\left((0, \infty)^{m}\right)$-valued Schwartz functions space. Then, $\mathcal{F}(f) \in S\left(\mathbb{R}, L^{p}\left((0, \infty)^{m}\right)\right)$; being $\mathcal{F}$ the $\left(L^{p}\left((0, \infty)^{m}\right)\right.$-valued) Fourier transform. By using Plancherel equality for Laguerre functions expansion we obtain

$$
\left\|R_{\alpha, 1}(a)\right\|_{L^{2}\left((0, \infty)^{m}\right) \rightarrow L^{2}\left((0, \infty)^{m}\right)} \leq 1, \quad a>0 .
$$

Let $a>0$. From 23 we get, for every $g \in C_{c}^{\infty}\left((0, \infty)^{m}\right)$,

$$
R_{\alpha, 1}(a)(g)(x)=\int_{0}^{\infty} R_{\alpha, 1}(x, y ; a) g(y) d y, \quad \text { a.e. } x \notin \text { supp } g .
$$

Moreover, we have that for $x, y \in(0, \infty)^{m}$ and $t>0$,

$$
\begin{gathered}
A_{\alpha_{1}}(a) \mathcal{W}_{t}^{\alpha}(x, y ; a)=A_{\alpha_{1}}(a) W_{t a}^{\alpha_{1}}\left(\sqrt{a} x_{1}, \sqrt{a} y_{1}\right) a^{m / 2} \prod_{j=2}^{m} W_{t a}^{\alpha_{j}}\left(\sqrt{a} x_{j}, \sqrt{a} y_{j}\right) \\
=a^{(m+1) / 2}\left(\frac{d}{d\left(\sqrt{a} x_{1}\right)}+\sqrt{a} x_{1}-\frac{\alpha_{1}+1 / 2}{\sqrt{a} x_{1}}\right)\left[W_{t a}^{\alpha_{1}}\left(\sqrt{a} x_{1}, \sqrt{a} y_{1}\right)\right] \prod_{j=2}^{m} W_{t a}^{\alpha_{j}}\left(\sqrt{a} x_{j}, \sqrt{a} y_{j}\right) \\
=a^{(m+1) / 2}\left[A_{\alpha_{1}}(1) W_{t a}^{\alpha_{1}}\left(\tilde{x}_{1}, \tilde{y}_{1}\right)\right]_{\mid \tilde{x}_{1}=\sqrt{a} x_{1}, \tilde{y}_{1}=\sqrt{a} y_{1}} \prod_{j=2}^{m} W_{t a}^{\alpha_{j}}\left(\sqrt{a} x_{j}, \sqrt{a} y_{j}\right) .
\end{gathered}
$$

Then,

$$
\begin{aligned}
R_{\alpha, 1}(x, y ; a) & =\frac{a^{(m+1) / 2}}{\sqrt{\pi}} \int_{0}^{\infty}\left[A_{\alpha_{1}}(1) W_{t a}^{\alpha_{1}}\left(\tilde{x}_{1}, \tilde{y}_{1}\right)\right] \prod_{j=2}^{m} W_{t a}^{\alpha_{j}}\left(\tilde{x}_{j}, \tilde{y}_{j}\right)_{\mid \tilde{x}=\sqrt{a} x, \tilde{y}=\sqrt{a} y} \frac{d t}{\sqrt{t}} \\
& =\frac{a^{m / 2}}{\sqrt{\pi}} \int_{0}^{\infty}\left[A_{\alpha_{1}}(1) W_{s}^{\alpha_{1}}\left(\tilde{x}_{1}, \tilde{y}_{1}\right)\right] \prod_{j=2}^{m} W_{s}^{\alpha_{j}}\left(\tilde{x}_{j}, \tilde{y}_{j}\right)_{\mid \tilde{x}=\sqrt{a} x, \tilde{y}=\sqrt{a} y \frac{d s}{\sqrt{s}}} \\
& =a^{m / 2} R_{\alpha, 1}(\sqrt{a} x, \sqrt{a} y ; 1), \quad x, y \in(0, \infty)^{m}, \quad x \neq y .
\end{aligned}
$$

Since $R_{\alpha, 1}(x, y ; 1)$ is a standard Calderón-Zygmund kernel [25, Proposition 3.1] we can obtain

$$
\left|R_{\alpha, 1}(x, y ; a)\right| \leq \frac{C}{|x-y|^{m}}, \quad x, y \in(0, \infty)^{m}, x \neq y,
$$

and

$$
\left|\nabla_{x} R_{\alpha, 1}(x, y ; a)\right|+\left|\nabla_{y} R_{\alpha}(x, y ; a)\right| \leq \frac{C}{|x-y|^{m+1}}, \quad x, y \in(0, \infty)^{m}, x \neq y,
$$

where the constant $C>0$ does not depend on $a$.

By using Calderón-Zygmund theory we conclude that $R_{\alpha, 1}$ can be extended from $L^{2}\left((0, \infty)^{m}\right) \cap$ $L^{p}\left((0, \infty)^{m}\right)$ to $L^{p}\left((0, \infty)^{m}\right)$ as a bounded operator from $L^{p}\left((0, \infty)^{m}\right)$ into itself (as it was said earlier) and

where $C>0$ does not depend on $a$.

$$
\left\|R_{\alpha, 1}(a)\right\|_{L^{p}\left((0, \infty)^{m}\right) \rightarrow L^{p}\left((0, \infty)^{m}\right)} \leq C
$$

We deduce that $R_{\alpha, 1}(|u|)(\mathcal{F}(f)(u)) \in L^{1}\left(\mathbb{R}, L^{p}\left((0, \infty)^{m}\right)\right)$ and then $\mathcal{F}^{-1}\left(R_{\alpha, 1}(|u|)(\mathcal{F}(f)(u))\right) \in$ $L^{\infty}\left(\mathbb{R}, L^{p}\left((0, \infty)^{m}\right)\right)$. Furthermore, we have that

$$
R_{\alpha, 1}(f)=\mathcal{F}^{-1}\left(R_{\alpha, 1}(|u|)(\mathcal{F}(f)(u))\right) .
$$


Indeed, suppose that $g \in L^{1}\left(\mathbb{R}, L^{p}\left((0, \infty)^{m}\right)\right)$. We understand $g$ as a function defined in $\mathbb{R} \times(0, \infty)^{m}$. Let $h \in L^{p^{\prime}}\left((0, \infty)^{m}\right)$, where $p^{\prime}=p /(p-1)$. By using some properties of the Bochner integral and Hölder inequality we get

$$
\begin{aligned}
\int_{(0, \infty)^{m}} h(z)\left(\int_{\mathbb{R}} g(y, \cdot) e^{-i x y} d y\right)(z) d z & =\int_{\mathbb{R}} \int_{(0, \infty)^{m}} h(z) g(y, z) d z e^{-i x y} d y \\
& =\int_{(0, \infty)^{m}} h(z) \int_{\mathbb{R}} g(y, z) e^{-i x y} d y d z, \quad x \in \mathbb{R} .
\end{aligned}
$$

Then, for every $x \in \mathbb{R}$,

$$
\left(\int_{\mathbb{R}} g(y, \cdot) e^{-i x y} d y\right)(z)=\int_{\mathbb{R}} g(y, z) e^{-i x y} d y, \quad \text { a.e. } z \in(0, \infty)^{m} .
$$

According to [45), since $L^{p}\left((0, \infty)^{m}\right)$ is a UMD Banach space, we use [34, Theorem 3.4] to show that $R_{\alpha, 1}$ defines a bounded operator from $L^{p}\left(\mathbb{R}, L^{p}\left((0, \infty)^{m}\right)\right)$ into itself. It is sufficient to see that the families of operators

$$
\left\{R_{\alpha, 1}(u)\right\}_{u>0} \text { and }\left\{u \frac{d}{d u} R_{\alpha, 1}(u)\right\}_{u>0}
$$

are $R$-bounded in $L^{p}\left((0, \infty)^{m}\right)$. It is well-known that if $\{T(u)\}_{u>0}$ is a set of bounded operators in $L^{p}\left((0, \infty)^{m}\right)$, then $\mathcal{T}=\{T(u)\}_{u>0}$ is $R$-bounded in $L^{p}\left((0, \infty)^{m}\right)$ if, and only if, there exists $C>0$ such that

$$
\left\|\left(\sum_{j=1}^{N}\left|T\left(u_{j}\right) g_{j}\right|^{2}\right)^{1 / 2}\right\|_{L^{p}\left((0, \infty)^{m}\right)} \leq C\left\|\left(\sum_{j=1}^{N}\left|g_{j}\right|^{2}\right)^{1 / 2}\right\|_{L^{p}\left((0, \infty)^{m}\right)^{\prime}},
$$

for every sequences $\left(u_{j}\right)_{j=1}^{N} \subset(0, \infty),\left(g_{j}\right)_{j=1}^{N} \subset L^{p}\left((0, \infty)^{m}\right)$ and $N \in \mathbb{N}$.

Suppose that $\{T(u)\}_{u>0}$ is a family of bounded operators in $L^{2}\left((0, \infty)^{m}\right)$ such that

$$
\sup _{u>0}\|T(u)\|_{\mathcal{L}\left(L^{2}\left((0, \infty)^{m}\right)\right)}<\infty
$$

being $\mathcal{L}\left(L^{2}\left((0, \infty)^{m}\right)\right)$ the space of bounded linear mappings from $L^{2}\left((0, \infty)^{m}\right)$ into itself. Moreover, assume that, for every $u>0$ and $g \in C_{c}^{\infty}\left((0, \infty)^{m}\right)$,

$$
T(u) g(x)=\int_{(0, \infty)^{m}} K_{u}(x, y) g(y) d y, \quad x \notin \operatorname{supp}(g),
$$

where

$$
\sup _{u>0}\left|K_{u}(x, y)\right| \leq \frac{C}{|x-y|^{m}}, \quad x, y \in(0, \infty)^{m}, x \neq y,
$$

and

$$
\sup _{u>0}\left(\left|\nabla_{x} K_{u}(x, y)\right|+\left|\nabla_{y} K_{u}(x, y)\right|\right) \leq \frac{C}{|x-y|^{m+1}}, \quad x, y \in(0, \infty)^{m}, x \neq y .
$$

Then, by [33, Theorem 1.3 Chapter XII], 46] holds for every $\left(u_{j}\right)_{j=1}^{N} \subset(0, \infty),\left(g_{j}\right)_{j=1}^{N} \subset L^{p}\left((0, \infty)^{m}\right)$ and $N \in \mathbb{N}$.

Lemma 3.3. Let $\alpha \in(-1 / 2, \infty)^{m}$. The family of operators $\left\{R_{\alpha, 1}(u)\right\}_{u>0}$ is $R$-bounded in $L^{p}\left((0, \infty)^{m}\right)$.

Proof. It is enough to take into account the above observation and [23), 42, ,43) and 444.

The proof of the differentiability of the map

$$
\begin{aligned}
(0, \infty) & \longrightarrow \mathcal{L}\left(L^{p}\left((0, \infty)^{m}\right)\right) \\
u & \longmapsto R_{\alpha, 1}(u)
\end{aligned}
$$

and the $R$-boundedness of the family $\left\{u \frac{d}{d u} R_{\alpha, 1}(u)\right\}_{u>0}$ are more involved. In order to show these last properties we will use some results established in [16].

Lemma 3.4. Let $\alpha \in[1 / 2, \infty)^{m}$. The function

$$
\begin{aligned}
R_{\alpha, 1}:(0, \infty) & \longrightarrow \mathcal{L}\left(L^{p}\left((0, \infty)^{m}\right)\right) \\
a & \longmapsto R_{\alpha, 1}(a)
\end{aligned}
$$

is differentiable. 
Proof. In [16] it was shown that the function

$$
\begin{aligned}
R_{1}:(0, \infty) & \longrightarrow \mathcal{L}\left(L^{p}\left(\mathbb{R}^{m}\right)\right) \\
a & \longmapsto R_{1}(a)
\end{aligned}
$$

is differentiable. By identifying $g \in L^{p}\left((0, \infty)^{m}\right)$ with $g_{0} \in L^{p}\left(\mathbb{R}^{m}\right)$ defined by

$$
g_{0}(x)= \begin{cases}g(x), & x_{j}>0, j=1, \ldots, m \\ 0, & x_{j} \leq 0, \text { for some } j=1, \ldots, m\end{cases}
$$

we have that

$$
\begin{aligned}
R_{1}:(0, \infty) & \longrightarrow \mathcal{L}\left(L^{p}\left((0, \infty)^{m}\right)\right) \\
a & \longmapsto R_{1}(a)
\end{aligned}
$$

is differentiable. In order to show that $R_{\alpha, 1}$ is differentiable we will prove that $D_{\alpha, 1}=R_{\alpha, 1}-R_{1}$ is differentiable. We divide this proof in three steps.

Step 1: In this first step we show that the operator $D_{\alpha, 1}(a)$ is bounded from $L^{p}\left((0, \infty)^{m}\right)$ into itself, for every $a>0$.

Let $a>0$ and $g \in L^{p}\left((0, \infty)^{m}\right)$. Recall that

$$
G_{t}^{\alpha_{1}}\left(x_{1}, y_{1}\right)=A_{\alpha_{1}}(1) W_{t}^{\alpha_{1}}\left(x_{1}, y_{1}\right), \quad x_{1}, y_{1}, t \in(0, \infty)
$$

and

$$
G_{t}\left(x_{1}, y_{1}\right)=A(1) W_{t}\left(x_{1}, y_{1}\right), \quad x_{1}, y_{1}, t \in(0, \infty)
$$

We can write

$$
\begin{aligned}
D_{\alpha, 1}(x, y ; a)= & R_{\alpha, 1}(x, y ; a)-R_{1}(x, y ; a)=a^{m / 2}\left[R_{\alpha, 1}(\sqrt{a} x, \sqrt{a} y ; 1)-R_{1}(\sqrt{a} x, \sqrt{a} y ; 1)\right] \\
= & \frac{a^{m / 2}}{\sqrt{\pi}} \int_{0}^{\infty}\left[\left(A_{\alpha_{1}}(1) \mathcal{W}_{t}^{\alpha}\right)(\sqrt{a} x, \sqrt{a} y)-\left(A_{1}(1) \mathcal{W}_{t}\right)(\sqrt{a} x, \sqrt{a} y)\right] \frac{d t}{\sqrt{t}} \\
= & \frac{a^{m / 2}}{\sqrt{\pi}}\left\{\int_{0}^{\infty}\left[G_{t}^{\alpha_{1}}-G_{t}\right]\left(\sqrt{a} x_{1}, \sqrt{a} y_{1}\right) \prod_{j=2}^{m} W_{t}^{\alpha_{j}}\left(\sqrt{a} x_{j}, \sqrt{a} y_{j}\right) \frac{d t}{\sqrt{t}}\right. \\
& +\sum_{i=2}^{m} \int_{0}^{\infty} G_{t}\left(\sqrt{a} x_{1}, \sqrt{a} y_{1}\right) \prod_{j=2}^{i-1} W_{t}\left(\sqrt{a} x_{j}, \sqrt{a} y_{j}\right) \\
& \left.\times\left[W_{t}^{\alpha_{i}}\left(\sqrt{a} x_{i}, \sqrt{a} y_{i}\right)-W_{t}\left(\sqrt{a} x_{i}, \sqrt{a} y_{i}\right)\right] \prod_{j=i+1}^{m} W_{t}^{\alpha_{j}}\left(\sqrt{a} x_{j}, \sqrt{a} y_{j}\right) \frac{d t}{\sqrt{t}}\right\} \\
= & \sum_{i=1}^{m} M_{i}(x, y ; a), \quad x, y \in(0, \infty)^{m} . \quad
\end{aligned}
$$

Note that we have apply the identity,

$$
\prod_{j=1}^{\ell} a_{j}-\prod_{j=1}^{\ell} b_{j}=\sum_{i=1}^{\ell}\left(\prod_{j=1}^{i-1} b_{j}\right)\left[a_{i}-b_{i}\right]\left(\prod_{j=i+1}^{m} a_{j}\right), \quad a_{j}, b_{j} \in \mathbb{R}, \ell \in \mathbb{N},
$$

which allows us to compare the terms of each product one by one.

We now prove that

$$
\int_{(0, \infty)^{m}}\left|D_{\alpha, 1}(x, y ; a)\right||g(y)| d y<\infty, \quad x \in(0, \infty)^{m}
$$


According to Lemma 3.2. (b), we have that

$$
\begin{aligned}
& a^{1 / 2} \int_{0}^{\infty}\left[G_{t}^{\alpha_{1}}-G_{t}\right]\left(\sqrt{a} x_{1}, \sqrt{a} y_{1}\right) \frac{d t}{\sqrt{t}} \\
& \quad \leq C\left\{\begin{array}{l}
\frac{1}{x_{1}}, \quad 0<y_{1} \leq \frac{x_{1}}{2}<\infty \\
\frac{1}{x_{1}}\left(1+\sqrt{\frac{x_{1}}{\left|x_{1}-y_{1}\right|}}\right), \quad 0<\frac{x_{1}}{2}<y_{1}<2 x_{1}<\infty \\
\frac{1}{y_{1}}, \quad 0<2 x_{1} \leq y_{1}<\infty
\end{array}\right.
\end{aligned}
$$

where $C>0$ does not depend on $a$.

From now on, to abbreviate notation, if $x=\left(x_{1}, \ldots, x_{m}\right) \in(0, \infty)^{m}$, we simply call $\bar{x}_{j}=$ $\left(x_{1}, \ldots, x_{j-1}, x_{j+1}, \ldots, x_{m}\right) \in(0, \infty)^{m-1}, j=1, \ldots, m$. Hence, from Lemma 3.1. $(a)$, we get

$$
\begin{aligned}
\int_{(0, \infty)^{m}} & M_{1}(x, y ; a)|| g(y) \mid d y \leq C\left[\int_{0}^{x_{1} / 2} \frac{1}{x_{1}}+\int_{x_{1} / 2}^{2 x_{1}} \frac{1}{x_{1}}\left(1+\sqrt{\frac{x_{1}}{\left|x_{1}-y_{1}\right|}}\right)+\int_{2 x_{1}}^{\infty} \frac{1}{y_{1}}\right] \\
& \times\left\{\sup _{t>0} \int_{(0, \infty)^{m-1}} a^{(m-1) / 2} \frac{e^{-c a\left|\bar{x}_{1}-\bar{y}_{1}\right|^{2} / t}}{t^{(m-1) / 2}}|g(y)| d \bar{y}_{1}\right\} d y_{1} .
\end{aligned}
$$

We consider the maximal operator

$$
\mathfrak{W}_{*}(h)(x)=\sup _{t>0}\left|\int_{(0, \infty)^{m-1}} \frac{e^{-c a|x-y|^{2} / t}}{t^{(m-1) / 2}} h(y) d y\right|, \quad x \in(0, \infty)^{m-1},
$$

which is bounded from $L^{p}\left((0, \infty)^{m-1}\right)$ into itself for each $1<p<\infty$.

Moreover, the Hardy operators [14, p. 244, (9.9.1) and (9.9.2)]

$$
H_{0}(F)(x)=\frac{1}{x} \int_{0}^{x} F(y) d y \quad \text { and } \quad H^{\infty} F(x)=\int_{x}^{\infty} \frac{F(y)}{y} d y, \quad x \in(0, \infty),
$$

and the operator

$$
N(F)(x)=\frac{1}{x} \int_{x / 2}^{2 x}\left(1+\sqrt{\frac{x}{|x-y|}}\right) F(y) d y, \quad x \in(0, \infty),
$$

are bounded from $L^{p}(0, \infty)$ into itself for each $1<p<\infty$. Then

$$
\int_{(0, \infty)^{m}}\left|M_{1}(x, y ; a)\right||g(y)| d y<\infty, \quad \text { a.e. } x \in(0, \infty)^{m} .
$$

Next we study $M_{2}$. From Lemma 3.1. (b), we obtain

$$
\begin{aligned}
& \int_{0}^{\infty}\left|W_{t}^{\alpha_{2}}\left(\sqrt{a} x_{2}, \sqrt{a} y_{2}\right)-W_{t}\left(\sqrt{a} x_{2}, \sqrt{a} y_{2}\right)\right| \frac{d t}{t} \leq C\left(\int_{0}^{x_{2} y_{2} a} \frac{1}{\sqrt{t} x_{2} y_{2} a} d t+\int_{x_{2} y_{2} a}^{\infty} \frac{1}{t^{3 / 2}} d t\right) \\
& \leq \frac{C}{\sqrt{x_{2} y_{2} a}}, \quad x_{2}, y_{2}, a \in(0, \infty),
\end{aligned}
$$

and

$$
\begin{gathered}
\int_{0}^{\infty}\left|W_{t}^{\alpha_{2}}\left(\sqrt{a} x_{2}, \sqrt{a} y_{2}\right)-W_{t}\left(\sqrt{a} x_{2}, \sqrt{a} y_{2}\right)\right| \frac{d t}{t} \leq C \int_{0}^{\infty} \frac{e^{-c a\left|x_{2}-y_{2}\right|^{2} / t}}{t^{3 / 2}} d t \\
\quad \leq \frac{C}{a^{1 / 2}\left|x_{2}-y_{2}\right|} \leq C \begin{cases}\frac{1}{\sqrt{a} x_{2}}, & 0<y_{2}<x_{2} / 2 \\
\frac{1}{\sqrt{a} y_{2}}, & 2 x_{2}<y_{2}<\infty\end{cases}
\end{gathered}
$$

The above estimates allow us to write, for every $x \in(0, \infty)^{m}$,

$$
\begin{aligned}
\int_{(0, \infty)^{m}} & M_{2}(x, y ; a) \| g(y) \mid d y \\
& \leq C a^{(m-1) / 2}\left(\int_{0}^{x_{2} / 2} \frac{1}{x_{2}}+\int_{x_{2} / 2}^{2 x_{2}} \frac{1}{x_{2}}+\int_{2 x_{2}}^{\infty} \frac{1}{y_{2}}\right)\left\{\sup _{t>0} \int_{(0, \infty)^{m-1}} \frac{e^{-c a\left|\bar{x}_{2}-\bar{y}_{2}\right|^{2} / t}}{t^{(m-1) / 2}}|g(y)| d \bar{y}_{2}\right\} d y_{2} .
\end{aligned}
$$


where $C$ is not depending on $a$.

So, as in the case of $M_{1}$, we conclude

$$
\int_{(0, \infty)^{m}}\left|M_{2}(x, y ; a)\right||g(y)| d y<\infty, \quad \text { a.e. } x \in(0, \infty)^{m} .
$$

In a similar way we can see that, for every $j=3, \ldots, m$,

$$
\int_{(0, \infty)^{m}}\left|M_{j}(x, y ; a)\right||g(y)| d y<\infty, \quad \text { a.e. } x \in(0, \infty)^{m} .
$$

Hence, (47) is justified. Thus, we can write

$$
D_{\alpha, 1}(a)(g)(x)=\int_{(0, \infty)^{m}}\left[R_{\alpha, 1}(x, y ; a)-R_{1}(x, y ; a)\right] g(y) d y, \quad \text { a.e. } x \in(0, \infty)^{m},
$$

where the last integral is absolutely convergent. Moreover, by using the $L^{p}$-boundedness of the auxiliar operators we conclude that

$$
\left\|D_{\alpha, 1}(a)(g)\right\|_{L^{p}\left((0, \infty)^{m}\right)} \leq C\|g\|_{L^{p}\left((0, \infty)^{m}\right)}, \quad g \in L^{p}\left((0, \infty)^{m}\right),
$$

where $C>0$ does not depend on $a$.

Step 2: The aim of this step is to show that

$$
\left\|d_{\alpha, 1}(a)(g)\right\|_{L^{p}\left((0, \infty)^{m}\right)} \leq \frac{C}{a}\|g\|_{L^{p}\left((0, \infty)^{m}\right)}, \quad a>0,
$$

being $d_{\alpha, 1}(a)$ the operator given by

$$
d_{\alpha, 1}(a)(g)(x)=\int_{(0, \infty)^{m}} \partial_{a}\left[R_{\alpha, 1}(x, y ; a)-R_{1}(x, y ; a)\right] g(y) d y .
$$

We now analyze $\partial_{a}\left[R_{\alpha, 1}(x, y ; a)-R_{1}(x, y ; a)\right], x, y \in(0, \infty)^{m}$. We have that

$$
\begin{aligned}
& \partial_{a}\left[R_{\alpha, 1}(x, y ; a)-R_{1}(x, y ; a)\right]=\partial_{a}\left(a^{m / 2}\left[R_{\alpha, 1}(\sqrt{a} x, \sqrt{a} y ; 1)-R_{1}(\sqrt{a} x, \sqrt{a} y ; 1)\right]\right) \\
& =\frac{m}{2} a^{(m-2) / 2}\left[R_{\alpha, 1}(\sqrt{a} x, \sqrt{a} y ; 1)-R_{1}(\sqrt{a} x, \sqrt{a} y ; 1)\right] \\
& +\frac{a^{(m-1) / 2}}{2} \int_{0}^{\infty}\left(x_{1}\left(\partial_{x_{1}} G_{t}^{\alpha_{1}}\right)\left(\sqrt{a} x_{1}, \sqrt{a} y_{1}\right) \prod_{j=2}^{m} W_{t}^{\alpha_{j}}\left(\sqrt{a} x_{j}, \sqrt{a} y_{j}\right)\right. \\
& +G_{t}^{\alpha_{1}}\left(\sqrt{a} x_{1}, \sqrt{a} y_{1}\right) \sum_{j=2}^{m} x_{j}\left(\partial_{x_{j}} W_{t}^{\alpha_{j}}\right)\left(\sqrt{a} x_{j}, \sqrt{a} y_{j}\right) \prod_{i=2, i \neq j}^{m} W_{t}^{\alpha_{i}}\left(\sqrt{a} x_{i}, \sqrt{a} y_{i}\right) \\
& +y_{1}\left(\partial_{y_{1}} G_{t}^{\alpha_{1}}\right)\left(\sqrt{a} x_{1}, \sqrt{a} y_{1}\right) \prod_{j=2}^{m} W_{t}^{\alpha_{j}}\left(\sqrt{a} x_{j}, \sqrt{a} y_{j}\right) \\
& +G_{t}^{\alpha_{1}}\left(\sqrt{a} x_{1}, \sqrt{a} y_{1}\right) \sum_{j=2}^{m} y_{j}\left(\partial_{y_{j}} W_{t}^{\alpha_{j}}\right)\left(\sqrt{a} x_{j}, \sqrt{a} y_{j}\right) \prod_{i=2, i \neq j}^{m} W_{t}^{\alpha_{i}}\left(\sqrt{a} x_{i}, \sqrt{a} y_{i}\right) \\
& -x_{1}\left(\partial_{x_{1}} G_{t}\right)\left(\sqrt{a} x_{1}, \sqrt{a} y_{1}\right) \prod_{j=2}^{m} W_{t}\left(\sqrt{a} x_{j}, \sqrt{a} y_{j}\right) \\
& -G_{t}\left(\sqrt{a} x_{1}, \sqrt{a} y_{1}\right) \sum_{j=2}^{m} x_{j}\left(\partial_{x_{j}} W_{t}\right)\left(\sqrt{a} x_{j}, \sqrt{a} y_{j}\right) \prod_{i=2, i \neq j}^{m} W_{t}\left(\sqrt{a} x_{i}, \sqrt{a} y_{i}\right) \\
& -y_{1}\left(\partial_{y_{1}} G_{t}\right)\left(\sqrt{a} x_{1}, \sqrt{a} y_{1}\right) \prod_{j=2}^{m} W_{t}\left(\sqrt{a} x_{j}, \sqrt{a} y_{j}\right) \\
& \left.-G_{t}\left(\sqrt{a} x_{1}, \sqrt{a} y_{1}\right) \sum_{j=2}^{m} y_{j}\left(\partial_{y_{j}} W_{t}\right)\left(\sqrt{a} x_{j}, \sqrt{a} y_{j}\right) \prod_{i=2, i \neq j}^{m} W_{t}\left(\sqrt{a} x_{i}, \sqrt{a} y_{i}\right)\right) \frac{d t}{\sqrt{\pi t}}
\end{aligned}
$$


According to 48 we get, for every $g \in L^{p}\left((0, \infty)^{m}\right)$,

$$
\begin{aligned}
& \left\|a^{(m-2) / 2} \int_{(0, \infty)^{m}}\left[R_{\alpha, 1}(\sqrt{a} x, \sqrt{a} y ; 1)-R_{1}(\sqrt{a} x, \sqrt{a} y ; 1)\right] g(y) d y\right\|_{L^{p}\left((0, \infty)^{m}\right)} \\
& \quad \leq \frac{C}{a}\|g\|_{L^{p}\left((0, \infty)^{m}\right)} .
\end{aligned}
$$

We want to obtain analogous $L^{p}$-estimates for the remaining terms in 49 . We are going to reorganize them in the new kernels $S_{t}$ and $T_{t}$ defined below (see (51) and (63)).

We consider, for every $x, y \in(0, \infty)^{m}$ and $t>0$, the kernel $S_{t}$ given by

$$
\begin{aligned}
& S_{t}(x, y ; a)=G_{t}^{\alpha_{1}}\left(\sqrt{a} x_{1}, \sqrt{a} y_{1}\right) \sum_{j=2}^{m}\left(x_{j} \partial_{x_{j}}+y_{j} \partial_{y_{j}}\right) W_{t}^{\alpha_{j}}\left(\sqrt{a} x_{j}, \sqrt{a} y_{j}\right) \prod_{i=2, i \neq j}^{m} W_{t}^{\alpha_{i}}\left(\sqrt{a} x_{i}, \sqrt{a} y_{i}\right) \\
& -G_{t}\left(\sqrt{a} x_{1}, \sqrt{a} y_{1}\right) \sum_{j=2}^{m}\left(x_{j} \partial_{x_{j}}+y_{j} \partial_{y_{j}}\right) W_{t}\left(\sqrt{a} x_{j}, \sqrt{a} y_{j}\right) \prod_{i=2, i \neq j}^{m} W_{t}\left(\sqrt{a} x_{i}, \sqrt{a} y_{i}\right) \\
& =\left[G_{t}^{\alpha_{1}}\left(\sqrt{a} x_{1}, \sqrt{a} y_{1}\right)-G_{t}\left(\sqrt{a} x_{1}, \sqrt{a} y_{1}\right)\right] \sum_{j=2}^{m}\left(x_{j} \partial_{x_{j}}+y_{j} \partial_{y_{j}}\right) W_{t}^{\alpha_{j}}\left(\sqrt{a} x_{j}, \sqrt{a} y_{j}\right) \\
& \times \prod_{i=2, i \neq j}^{m} W_{t}^{\alpha_{i}}\left(\sqrt{a} x_{i}, \sqrt{a} y_{i}\right) \\
& +G_{t}\left(\sqrt{a} x_{1}, \sqrt{a} y_{1}\right) \sum_{j=2}^{m}\left(x_{j} \partial_{x_{j}}+y_{j} \partial_{y_{j}}\right)\left[W_{t}^{\alpha_{j}}\left(\sqrt{a} x_{j}, \sqrt{a} y_{j}\right)-W_{t}\left(\sqrt{a} x_{j}, \sqrt{a} y_{j}\right)\right] \\
& \times \prod_{i=2, i \neq j}^{m} W_{t}^{\alpha_{i}}\left(\sqrt{a} x_{i}, \sqrt{a} y_{i}\right) \\
& +G_{t}\left(\sqrt{a} x_{1}, \sqrt{a} y_{1}\right) \sum_{j=2}^{m}\left(x_{j} \partial_{x_{j}}+y_{j} \partial_{y_{j}}\right) W_{t}\left(\sqrt{a} x_{j}, \sqrt{a} y_{j}\right) \\
& \times \sum_{k=2, k \neq j}^{m}\left[W_{t}^{\alpha_{k}}\left(\sqrt{a} x_{k}, \sqrt{a} y_{k}\right)-W_{t}\left(\sqrt{a} x_{k}, \sqrt{a} y_{k}\right)\right] \prod_{s=2, s \neq j}^{k-1} W_{t}\left(\sqrt{a} x_{s}, \sqrt{a} y_{s}\right) \\
& \times \prod_{s=k+1, s \neq j}^{m} W_{t}^{\alpha_{s}}\left(\sqrt{a} x_{s}, \sqrt{a} y_{s}\right) \\
& =S_{t}^{1}(x, y ; a)+S_{t}^{2}(x, y ; a)+S_{t}^{3}(x, y ; a)
\end{aligned}
$$

We study each of the above terms separately. First of all we analyze $S_{t}^{1}$. Lemma 3.2. (b), with $\varepsilon=1 / 4$, implies that

$$
\begin{aligned}
\int_{0}^{\infty} \mid G_{t}^{\alpha_{1}} & \left(\sqrt{a} x_{1}, \sqrt{a} y_{1}\right)-G_{t}\left(\sqrt{a} x_{1}, \sqrt{a} y_{1}\right) \mid e^{5 t / 4} \frac{d t}{\sqrt{t}} \\
\leq & \frac{C}{\sqrt{a x_{1}}} \int_{0}^{\infty} \frac{e^{-c a\left|x_{1}-y_{1}\right|^{2} / t}}{t^{5 / 4}} d t \leq \frac{C}{\sqrt{a}} \frac{1}{x_{1}} \sqrt{\frac{x_{1}}{\left|x_{1}-y_{1}\right|}}, \quad 0<x_{1} / 2<y_{1}<2 x_{1}
\end{aligned}
$$

and

$$
\begin{aligned}
& \int_{0}^{\infty}\left|G_{t}^{\alpha_{1}}\left(\sqrt{a} x_{1}, \sqrt{a} y_{1}\right)-G_{t}\left(\sqrt{a} x_{1}, \sqrt{a} y_{1}\right)\right| e^{5 t / 4} \frac{d t}{\sqrt{t}} \\
& \quad \leq C \sqrt{a} \max \left\{x_{1}, y_{1}\right\} \int_{0}^{\infty} \frac{e^{-c a\left|x_{1}-y_{1}\right|^{2} / t}}{t^{2}} d t \leq \frac{C}{\sqrt{a}} \frac{\max \left\{x_{1}, y_{1}\right\}}{\left|x_{1}-y_{1}\right|^{2}} \leq \frac{C}{\sqrt{a}}\left\{\begin{array}{l}
\frac{1}{x_{1}}, \quad 0<y_{1}<x_{1} / 2 \\
\frac{1}{y_{1}}, \quad 0<2 x_{1}<y_{1}
\end{array}\right.
\end{aligned}
$$


By combining Lemma 3.1. $(a)$ and $(e)$ taken with $\varepsilon=1 / 4 ; 52$ and 53 we deduce that, for every $g \in L^{p}\left((0, \infty)^{m}\right), 1<p<\infty$ and $j=2, \ldots, m$,

$$
\begin{aligned}
& \mid \int_{(0, \infty)^{m}} g(y) \int_{0}^{\infty}\left[G_{t}^{\alpha_{1}}\left(\sqrt{a} x_{1}, \sqrt{a} y_{1}\right)-G_{t}\left(\sqrt{a} x_{1}, \sqrt{a} y_{1}\right)\right] \prod_{i=2, i \neq j}^{m} W_{t}^{\alpha_{i}}\left(\sqrt{a} x_{i}, \sqrt{a} y_{i}\right) \\
& \quad \times\left[x_{j}\left(\partial_{x_{j}} W_{t}^{\alpha_{j}}\right)\left(\sqrt{a} x_{j}, \sqrt{a} y_{j}\right)+y_{j}\left(\partial_{y_{j}} W_{t}^{\alpha_{j}}\right)\left(\sqrt{a} x_{j}, \sqrt{a} y_{j}\right)\right] \frac{d t}{\sqrt{t}} d y \mid \\
& \quad \leq \frac{C}{a}\left(\int_{0}^{x_{1} / 2} \frac{1}{x_{1}}+\int_{x_{1} / 2}^{2 x_{1}} \frac{1}{x_{1}} \sqrt{\frac{x_{1}}{\left|x_{1}-y_{1}\right|}}+\int_{2 x_{1}}^{\infty} \frac{1}{y_{1}}\right)\left\{\sup _{t>0} \int_{(0, \infty)^{m-1}} \frac{e^{-c a\left|\bar{x}_{1}-\bar{y}_{1}\right|^{2} / t}}{t^{(m-1) / 2}}|g(y)| d \bar{y}_{1}\right\} d y_{1}
\end{aligned}
$$

and the $L^{p}$-boundedness property for the Hardy and maximal operators leads to

$$
a^{(m-1) / 2}\left\|\int_{(0, \infty)^{m}} g(y) \int_{0}^{\infty} S_{t}^{1}(x, y ; a) \frac{d t}{\sqrt{t}} d y\right\|_{L^{p}\left((0, \infty)^{m}\right)} \leq \frac{C}{a}\|g\|_{L^{p}\left((0, \infty)^{m}\right)},
$$

where $C>0$ does not depend on $a$.

Now we pass to the study of the kernel $S_{t}^{3}$. Let $k=2, \ldots, m$. Lemma 3.1, (b), allows us to write

$$
\begin{aligned}
\int_{0}^{\infty} \mid W_{t}^{\alpha_{k}} & \left(\sqrt{a} x_{k}, \sqrt{a} y_{k}\right)-W_{t}\left(\sqrt{a} x_{k}, \sqrt{a} y_{k}\right) \mid \frac{d t}{t} \\
& \leq C \int_{0}^{\infty} \frac{e^{-t / 2}}{\left(\mathfrak{a}(t) a x_{k} y_{k}\right)^{1 / 4}} \frac{e^{-c a\left|x_{k}-y_{k}\right|^{2} / t}}{t^{3 / 2}} d t \leq \frac{C}{\left(a x_{k} y_{k}\right)^{1 / 4}} \int_{0}^{\infty} \frac{e^{-c a\left|x_{k}-y_{k}\right|^{2} / t}}{t^{5 / 4}} d t \\
& \leq \frac{C}{\sqrt{a}} \frac{1}{x_{k}} \sqrt{\frac{x_{k}}{\left|x_{k}-y_{k}\right|}}, \quad x_{k} / 2<y_{k}<2 x_{k},
\end{aligned}
$$

and from Lemma 3.1 . (a), we obtain

$$
\begin{aligned}
\int_{0}^{\infty}\left|W_{t}^{\alpha_{k}}\left(\sqrt{a} x_{k}, \sqrt{a} y_{k}\right)-W_{t}\left(\sqrt{a} x_{k}, \sqrt{a} y_{k}\right)\right| \frac{d t}{t} \\
\quad \leq C \int_{0}^{\infty} \frac{e^{-c a\left|x_{k}-y_{k}\right|^{2} / t}}{t^{3 / 2}} d t \leq \frac{C}{\sqrt{a}} \frac{1}{\left|x_{k}-y_{k}\right|} \leq \frac{C}{\sqrt{a}}\left\{\begin{array}{l}
\frac{1}{x_{k}}, \quad 0<y_{k}<x_{k} / 2, \\
\frac{1}{y_{k}}, \quad 0<2 x_{k}<y_{k} .
\end{array}\right.
\end{aligned}
$$

By combining Lemma 3.1. $(a)$ and $(d)$; Lemma 3.2, $(a)$; (55) and (56) we deduce

$$
\begin{array}{rl}
\mid \int_{(0, \infty)^{m}} & g(y) \int_{0}^{\infty} G_{t}\left(\sqrt{a} x_{1}, \sqrt{a} y_{1}\right) \sum_{j=2}^{m}\left(x_{j} \partial_{x_{j}}+y_{j} \partial_{y_{j}}\right) W_{t}\left(\sqrt{a} x_{j}, \sqrt{a} y_{j}\right) \\
& \times \sum_{k=2, k \neq j}^{m}\left[W_{t}^{\alpha_{k}}\left(\sqrt{a} x_{k}, \sqrt{a} y_{k}\right)-W_{t}\left(\sqrt{a} x_{k}, \sqrt{a} y_{k}\right)\right] \prod_{s=2, s \neq j}^{k-1} W_{t}\left(\sqrt{a} x_{s}, \sqrt{a} y_{s}\right) \\
& \times \prod_{s=k+1, s \neq j}^{m} W_{t}^{\alpha_{s}}\left(\sqrt{a} x_{s}, \sqrt{a} y_{s}\right) \frac{d t}{\sqrt{t}} d y \mid \\
\leq & \frac{C}{a} \sum_{j=2}^{m} \sum_{k=2, k \neq j}^{m}\left(\int_{0}^{x_{k} / 2} \frac{1}{x_{k}}+\int_{x_{k} / 2}^{2 x_{k}} \frac{1}{x_{k}} \sqrt{\frac{x_{k}}{\left|x_{k}-y_{k}\right|}}+\int_{2 x_{k}}^{\infty} \frac{1}{y_{k}}\right) \\
& \times\left\{\sup _{t>0} \int_{(0, \infty)^{m-1}} \frac{e^{-c a\left|\bar{x}_{k}-\bar{y}_{k}\right|^{2} / t}}{t^{(m-1) / 2}}|g(y)| d \bar{y}_{k}\right\} d y_{k}, \quad x \in(0, \infty)^{m} .
\end{array}
$$

Then, for every $g \in L^{p}\left((0, \infty)^{m}\right)$,

$$
a^{(m-1) / 2}\left\|\int_{(0, \infty)^{m}} g(y) \int_{0}^{\infty} S_{t}^{3}(x, y ; a) \frac{d t}{\sqrt{t}} d y\right\|_{L^{p}\left((0, \infty)^{m}\right)} \leq \frac{C}{a}\|g\|_{L^{p}\left((0, \infty)^{m}\right)}
$$

where $C>0$ does not depend on $a$. 
Next we concentrate on the kernel $S_{t}^{2}$. Lemma 3.1, $(f)$, give us

$$
\begin{gathered}
\int_{0}^{\infty}\left|x_{j}\left[\partial_{x_{j}}\left(W_{t}^{\alpha_{j}}-W_{t}\right)\right]\left(\sqrt{a} x_{j}, \sqrt{a} y_{j}\right)+y_{j}\left[\partial_{y_{j}}\left(W_{t}^{\alpha_{j}}-W_{t}\right)\right]\left(\sqrt{a} x_{j}, \sqrt{a} y_{j}\right)\right| \frac{d t}{t} \\
\leq \frac{C}{a} \frac{1}{\left(x_{j} y_{j}\right)^{1 / 4}\left|x_{j}-y_{j}\right|^{1 / 2}}, \quad x_{j}, y_{j} \in(0, \infty) .
\end{gathered}
$$

Also, from Lemma 3.1. $(d)$ and $(e)$, we get

$$
\begin{gathered}
\int_{0}^{\infty}\left|x_{j}\left[\partial_{x_{j}}\left(W_{t}^{\alpha_{j}}-W_{t}\right)\right]\left(\sqrt{a} x_{j}, \sqrt{a} y_{j}\right)+y_{j}\left[\partial_{y_{j}}\left(W_{t}^{\alpha_{j}}-W_{t}\right)\right]\left(\sqrt{a} x_{j}, \sqrt{a} y_{j}\right)\right| \frac{d t}{t} \\
\leq \frac{C}{a} \frac{1}{\left|x_{j}-y_{j}\right|} \leq \frac{C}{\sqrt{a}}\left\{\begin{array}{c}
\frac{1}{x_{j}}, 0<y_{j}<x_{j} / 2, \\
\frac{1}{y_{j}}, \quad 0<2 x_{j}<y_{j} .
\end{array}\right.
\end{gathered}
$$

By combining Lemma 3.1. (a); Lemma 3.2. (a); (59) and 60 we obtain, for every $g \in L^{p}\left((0, \infty)^{m}\right)$, $1<p<\infty$,

$$
\begin{aligned}
& \mid \int_{(0, \infty)^{m}} g(y) \int_{0}^{\infty} G_{t}\left(\sqrt{a} x_{1}, \sqrt{a} y_{1}\right) \sum_{j=2}^{m}\left(x_{j} \partial_{x_{j}}+y_{j} \partial_{y_{j}}\right)\left[W_{t}^{\alpha_{j}}\left(\sqrt{a} x_{j}, \sqrt{a} y_{j}\right)-W_{t}\left(\sqrt{a} x_{j}, \sqrt{a} y_{j}\right)\right] \\
& \quad \times \prod_{i=2, i \neq j}^{m} W_{t}^{\alpha_{i}}\left(\sqrt{a} x_{i}, \sqrt{a} y_{i}\right) \frac{d t}{\sqrt{t}} d y \mid \\
& \leq \frac{C}{a} \sum_{j=2}^{m}\left(\int_{0}^{x_{j} / 2} \frac{1}{x_{j}}+\int_{x_{j} / 2}^{2 x_{j}} \frac{1}{x_{j}} \sqrt{\frac{x_{j}}{\left|x_{j}-y_{j}\right|}}+\int_{2 x_{j}}^{\infty} \frac{1}{y_{j}}\right)\left\{\sup _{t>0} \int_{(0, \infty)^{m-1}} \frac{e^{-c a\left|\bar{x}_{j}-\bar{y}_{j}\right|^{2} / t}}{t^{(m-1) / 2}}|g(y)| d \bar{y}_{j}\right\} d y_{j}
\end{aligned}
$$

$L^{p}$-boundedness properties of the Hardy and maximal operators lead to

$$
a^{(m-1) / 2}\left\|\int_{(0, \infty)^{m}} g(y) \int_{0}^{\infty} S_{t}^{2}(x, y ; a) \frac{d t}{\sqrt{t}} d y\right\|_{L^{p}\left((0, \infty)^{m}\right)} \leq \frac{C}{a}\|g\|_{L^{p}\left((0, \infty)^{m}\right)},
$$

for every $g \in L^{p}\left((0, \infty)^{m}\right), 1<p<\infty$. Here $C>0$ is not depending on $a$.

Putting together (54), (58) and (61) we obtain

$$
a^{(m-1) / 2}\left\|\int_{(0, \infty)^{m}} g(y) \int_{0}^{\infty} S_{t}(x, y ; a) \frac{d t}{\sqrt{t}} d y\right\|_{L^{p}\left((0, \infty)^{m}\right)} \leq \frac{C}{a}\|g\|_{L^{p}\left((0, \infty)^{m}\right)},
$$

for every $g \in L^{p}\left((0, \infty)^{m}\right), 1<p<\infty$, where $C>0$ does not depend on $a$.

We now define the kernel

$$
\begin{aligned}
T_{t}(x, y ; a)= & \left(x_{1}\left[\partial_{x_{1}}\left(G_{t}^{\alpha_{1}}-G_{t}\right)\right]\left(\sqrt{a} x_{1}, \sqrt{a} y_{1}\right)+y_{1}\left[\partial_{y_{1}}\left(G_{t}^{\alpha_{1}}-G_{t}\right)\right]\left(\sqrt{a} x_{1}, \sqrt{a} y_{1}\right)\right) \\
& \times \prod_{j=2}^{m} W_{t}^{\alpha_{j}}\left(\sqrt{a} x_{j}, \sqrt{a} y_{j}\right) \\
& +\left(x_{1} \partial_{x_{1}} G_{t}\left(\sqrt{a} x_{1}, \sqrt{a} y_{1}\right)+y_{1} \partial_{y_{1}} G_{t}\left(\sqrt{a} x_{1}, \sqrt{a} y_{1}\right)\right) \\
& \times\left(\prod_{j=2}^{m} W_{t}^{\alpha_{j}}\left(\sqrt{a} x_{j}, \sqrt{a} y_{j}\right)-\prod_{j=2}^{m} W_{t}\left(\sqrt{a} x_{j}, \sqrt{a} y_{j}\right)\right) \\
= & T_{t}^{1}(x, y ; a)+T_{t}^{2}(x, y ; a), \quad x, y \in(0, \infty)^{m} \text { and } t>0 .
\end{aligned}
$$


We start with $T_{t}^{1}$. By Lemma 3.2, $(d)$ and $(e)$, it follows that

$$
\begin{aligned}
& \int_{0}^{\infty}\left|x_{1} \partial_{x_{1}}\left(G_{t}-G_{t}^{\alpha_{1}}\right)\left(\sqrt{a} x_{1}, \sqrt{a} y_{1}\right)+y_{1} \partial_{y_{1}}\left(G_{t}-G_{t}^{\alpha_{1}}\right)\left(\sqrt{a} x_{1}, \sqrt{a} y_{1}\right)\right| \frac{d t}{\sqrt{t}} \\
& \quad \leq \frac{C}{a}\left[\frac{1}{\left|x_{1}-y_{1}\right|^{1 / 2}}\left(\frac{x_{1}^{1 / 4}}{y_{1}^{3 / 4}}+\frac{y_{1}^{1 / 4}}{x_{1}^{3 / 4}}\right)+\frac{1}{\sqrt{x_{1}^{2}+y_{1}^{2}}}\right] \leq \frac{C}{a} \frac{1}{x_{1}}\left(1+\sqrt{\frac{x_{1}}{\left|x_{1}-y_{1}\right|}}\right), \quad x_{1} / 2<y_{1}<2 x_{1},
\end{aligned}
$$

and from Lemma $3.2(f)$, we deduce that

$$
\begin{aligned}
& \int_{0}^{\infty}\left|x_{1} \partial_{x_{1}}\left(G_{t}-G_{t}^{\alpha \alpha_{1}}\right)\left(\sqrt{a} x_{1}, \sqrt{a} y_{1}\right)+y_{1} \partial_{y_{1}}\left(G_{t}-G_{t}^{\alpha_{1}}\right)\left(\sqrt{a} x_{1}, \sqrt{a} y_{1}\right)\right| \frac{d t}{\sqrt{t}} \\
& \quad \leq \frac{C}{\sqrt{a}} \int_{0}^{\infty} \frac{e^{-c a \max \left\{x_{1}, y_{1}\right\}^{2} / t}}{t^{3 / 2}} d t \leq \frac{C}{a}\left\{\begin{array}{l}
\frac{1}{x_{1}}, 0<y_{1}<x_{1} / 2 . \\
\frac{1}{y_{1}}, 0<2 x_{1}<y_{1} .
\end{array}\right.
\end{aligned}
$$

According to Lemma 3.1. (a); (64) and 65) it follows that, for every $g \in L^{p}\left((0, \infty)^{m}\right), 1<p<\infty$ and $x \in(0, \infty)^{m}$,

$$
\begin{aligned}
\left|\int_{(0, \infty)^{m}} g(y) \int_{0}^{\infty} T_{t}^{1}(x, y, a) \frac{d t}{\sqrt{t}} d y\right| \leq & \frac{C}{a}\left(\int_{0}^{x_{1} / 2} \frac{1}{x_{1}}+\int_{x_{1} / 2}^{2 x_{1}} \frac{1}{x_{1}}\left(1+\sqrt{\frac{x_{1}}{\left|x_{1}-y_{1}\right|}}\right)+\int_{2 x_{1}}^{\infty} \frac{1}{y_{1}}\right) \\
& \times\left\{\sup _{t>0} \int_{(0, \infty)^{m-1}} \frac{e^{-c a\left|\bar{x}_{1}-\bar{y}_{1}\right|^{2} / t}}{t^{(m-1) / 2}}|g(y)| d \bar{y}_{1}\right\} d y_{1} .
\end{aligned}
$$

And, $L^{p}$-boundedness properties of Hardy and maximal operators, together with, Jensen inequality, imply that

$$
a^{(m-1) / 2}\left\|\int_{(0, \infty)^{m}} g(y) \int_{0}^{\infty} T_{t}^{1}(x, y, a) \frac{d t}{\sqrt{t}} d y\right\|_{L^{p}\left((0, \infty)^{m}\right)} \leq \frac{C}{a}\|g\|_{L^{p}\left((0, \infty)^{m}\right)},
$$

where $C>0$ does not depend on $a$.

Now, we consider the kernel $T_{t}^{2}$. First of all, observe that

$$
\begin{aligned}
T_{t}^{2}(x, y ; a)= & \left(x_{1} \partial_{x_{1}} G_{t}\left(\sqrt{a} x_{1}, \sqrt{a} y_{1}\right)+y_{1} \partial_{y_{1}} G_{t}\left(\sqrt{a} x_{1}, \sqrt{a} y_{1}\right)\right) \\
& \times \sum_{i=2}^{m} \prod_{j=2}^{i-1} W_{t}\left(\sqrt{a} x_{j}, \sqrt{a} y_{j}\right)\left[W_{t}^{\alpha_{i}}\left(\sqrt{a} x_{i}, \sqrt{a} y_{i}\right)-W_{t}\left(\sqrt{a} x_{i}, \sqrt{a} y_{i}\right)\right] \\
& \times \prod_{j=i+1}^{m} W_{t}^{\alpha_{j}}\left(\sqrt{a} x_{j}, \sqrt{a} y_{j}\right) .
\end{aligned}
$$

By taking in mind Lemma 3.1. (a); Lemma 3.2. (c); 55), 56 and proceeding as in (57) we conclude, for every $g \in L^{p}\left((0, \infty)^{m}\right)$,

$$
a^{(m-1) / 2}\left\|\int_{(0, \infty)^{m}} g(y) \int_{0}^{\infty} T_{t}^{2}(x, y ; a) \frac{d t}{\sqrt{t}} d y\right\|_{L^{p}\left((0, \infty)^{m}\right)} \leq \frac{C}{a}\|g\|_{L^{p}\left((0, \infty)^{m}\right)},
$$

where $C>0$ does not depend on $a$.

Hence, 66) and (67) give us

$$
a^{(m-1) / 2}\left\|\int_{(0, \infty)^{m}} g(y) \int_{0}^{\infty} T_{t}(x, y ; a) \frac{d t}{\sqrt{t}} d y\right\|_{L^{p}\left((0, \infty)^{m}\right)} \leq \frac{C}{a}\|g\|_{L^{p}\left((0, \infty)^{m}\right)},
$$

being the constant $C>0$ independent of $a$.

By combining (50), 62 and (68) we conclude that, for every $g \in L^{p}\left((0, \infty)^{m}\right), 1<p<\infty$

$$
\left\|\int_{(0, \infty)^{m}} \partial_{a}\left(R_{\alpha, 1}(x, y ; a)-R_{1}(x, y ; a)\right) g(y) d y\right\|_{L^{p}\left((0, \infty)^{m}\right)} \leq \frac{C}{a}\|g\|_{L^{p}\left((0, \infty)^{m}\right)},
$$


Here, as above, $C>0$ does not depend on $a$. By proceeding in a similar way we can also obtain

$$
\left\|\int_{(0, \infty)^{m}} \partial_{a}^{2}\left(R_{\alpha, 1}(x, y ; a)-R_{1}(x, y ; a)\right) g(y) d y\right\|_{L^{p}\left((0, \infty)^{m}\right)} \leq \frac{C}{a^{3 / 2}}\|g\|_{L^{p}\left((0, \infty)^{m}\right)},
$$

for every $g \in L^{p}\left((0, \infty)^{m}\right), 1<p<\infty$, where $C>0$ is not depending on $a$.

Step 3: This last step is devoted to show the differentiability of the operator $D_{\alpha, 1}$.

Let $g \in L^{p}\left((0, \infty)^{m}\right), 1<p<\infty$. We can write

$$
\begin{aligned}
& \frac{1}{h}\left[D_{\alpha, 1}(a+h)(g)(x)-D_{\alpha .1}(a)(g)(x)\right]-d_{\alpha, 1}(a)(g)(x) \\
& \quad=\int_{(0, \infty)^{m}}\left[\frac{1}{h} \int_{a}^{a+h} \partial_{\lambda}\left(R_{\alpha, 1}(x, y ; \lambda)-R_{1}(x, y ; \lambda)\right) d \lambda-\partial_{a}\left(R_{\alpha, 1}(x, y ; a)-R_{1}(x, y ; a)\right)\right] g(y) d y \\
& \quad=\int_{(0, \infty)^{m}} \frac{1}{h} \int_{a}^{a+h} \int_{a}^{\lambda} \partial_{z}^{2}\left(R_{\alpha, 1}(x, y ; z)-R_{1}(x, y ; z)\right) d z d \lambda g(y) d y, \quad 0<|h|<a x \in(0, \infty)^{m}
\end{aligned}
$$

being $d_{\alpha, 1}(a)$ the operator considered in Step 2. According to 70 we get

$$
\begin{aligned}
& \left\|\frac{D_{\alpha, 1}(a+h)(g)-D_{\alpha, 1}(a)(g)}{h}-d_{\alpha, 1}(a)(g)\right\|_{L^{p}\left((0, \infty)^{m}\right)} \\
& =\left\|\frac{1}{h} \int_{a}^{a+h} \int_{a}^{\lambda} \int_{(0, \infty)^{m}} \partial_{z}^{2}\left(R_{\alpha, 1}(x, y ; z)-R_{1}(x, y ; z)\right) g(y) d y d z d \lambda\right\|_{L^{p}\left((0, \infty)^{m}\right)} \\
& \leq\left|\frac{1}{h} \int_{a}^{a+h} \int_{a}^{\lambda}\left\|\int_{(0, \infty)^{m}} \partial_{z}^{2}\left(R_{\alpha, 1}(x, y ; z)-R_{1}(x, y ; z)\right) g(y) d y\right\|_{L^{p}\left((0, \infty)^{m}\right)} d z d \lambda\right| \\
& \leq C\|g\|_{L^{p}\left((0, \infty)^{m}\right)}\left|\frac{1}{h} \int_{a}^{a+h} \int_{a}^{\lambda} \frac{d z d \lambda}{z^{3 / 2}}\right| \\
& \leq C\left|\frac{h-2 \sqrt{a^{2}+a h}+2 a}{\sqrt{a} h}\right|\|g\|_{L^{p}\left((0, \infty)^{m}\right)}, \quad 0<|h|<a .
\end{aligned}
$$

Hence,

$$
\lim _{h \rightarrow 0} \frac{D_{\alpha, 1}(a+h)-D_{\alpha, 1}(a)}{h}=d_{\alpha, 1}(a)
$$

in the sense of convergence in $\mathcal{L}\left(L^{p}\left((0, \infty)^{m}\right)\right)$.

We can proceed in a similar way when $a<0$. We conclude that for each $1<p<\infty$ the function

$$
\begin{aligned}
\mathbb{R} \backslash\{0\} & \longrightarrow \mathcal{L}\left(L^{p}\left((0, \infty)^{m}\right)\right) \\
a & \longmapsto R_{\alpha, 1}(a)
\end{aligned}
$$

is differentiable and that, for every $g \in L^{p}\left((0, \infty)^{m}\right)$,

$$
\left[\frac{d}{d a} R_{\alpha, 1}(a)\right] g(x)=\lim _{\varepsilon \rightarrow 0^{+}} \int_{|x-y|>\varepsilon} \partial_{a} R_{\alpha, 1}(x, y ; a) g(y) d y, \quad \text { a.e. } x \in(0, \infty)^{m} .
$$

Lemma 3.5. Let $\alpha \in(1 / 2, \infty)^{m}$ and $1<p<\infty$. The family of operators $\left\{a \frac{d}{d a} R_{\alpha, 1}(a)\right\}_{a \in \mathbb{R} \backslash\{0\}}$ is $R$-bounded in $L^{p}\left((0, \infty)^{m}\right)$.

Proof. From (69) and [16, Theorem 2.1] we deduce that

$$
\sup _{a \in \mathbb{R} \backslash\{0\}}\left\|a \frac{d}{d a} R_{\alpha, 1}(a)\right\|_{\mathcal{L}\left(L^{2}\left((0, \infty)^{m}\right)\right)}<\infty .
$$

Our next objective is to see that

$$
\sup _{a \in \mathbb{R} \backslash\{0\}}\left|a \frac{d}{d a}\left(R_{\alpha, 1}(x, y ; a)-R_{1}(x, y ; a)\right)\right| \leq \frac{C}{|x-y|^{m}}, \quad x, y \in(0, \infty)^{m}, x \neq y .
$$

We will have 490 in mind. Let $a>0$. In the sequel the constant $C>0$ does not depend on $a$. As in [43] and by [30] we get

$$
\left|a^{(m-2) / 2}\left(R_{\alpha, 1}(\sqrt{a} x, \sqrt{a} y ; 1)-R_{1}(\sqrt{a} x, \sqrt{a} y ; 1)\right)\right| \leq \frac{C}{a|x-y|^{m}}, \quad x, y \in(0, \infty)^{m}, x \neq y .
$$


Next we analyze the terms associates to $S_{t}$ and $T_{t}$ in 490 (see (51) and (63)).

By Lemma 3.1. (a); as in Lemma 3.1. (e); and Lemma 3.2, (b), we obtain

$$
\begin{aligned}
& \int_{0}^{\infty}\left|S_{t}^{1}(x, y ; a)\right| \frac{d t}{\sqrt{t}} \\
& \quad \leq C\left(\chi_{\left\{x_{1} / 2<y_{1}<2 x_{1}\right\}}\left(y_{1}\right) \int_{0}^{\infty} \frac{e^{-c a|x-y|^{2} / t}}{\sqrt{a} t^{(m+2) / 2}} d t+\max \left\{x_{1}, y_{1}\right\} \chi_{\left\{x_{1} / 2<y_{1}<2 x_{1}\right\}^{c}}\left(y_{1}\right) \int_{0}^{\infty} \frac{e^{-c a|x-y|^{2} / t}}{t^{2+(m-1) / 2}} d t\right) \\
& \quad \leq C\left(\frac{1}{a^{(m+1) / 2}|x-y|^{m}} \chi_{\left\{x_{1} / 2<y_{1}<2 x_{1}\right\}}\left(y_{1}\right)+\frac{\max \left\{x_{1}, y_{1}\right\}}{|x-y|^{m} a^{m / 2}} \int_{0}^{\infty} \frac{e^{-c a\left|x_{1}-y_{1}\right|^{2} / t}}{t^{3 / 2}} d t \chi_{\left\{x_{1} / 2<y_{1}<2 x_{1}\right\}^{c}}\left(y_{1}\right)\right) \\
& \quad \leq C\left(\frac{1}{a^{(m+1) / 2}|x-y|^{m}} \chi_{\left\{x_{1} / 2<y_{1}<2 x_{1}\right\}}\left(y_{1}\right)+\frac{\max \left\{x_{1}, y_{1}\right\}}{|x-y|^{m} a^{m / 2}} \frac{1}{\left|x_{1}-y_{1}\right| \sqrt{a}} \chi_{\left\{x_{1} / 2<y_{1}<2 x_{1}\right\}^{c}}\left(y_{1}\right)\right) \\
& \quad \leq \frac{C}{a^{(m+1) / 2}|x-y|^{m}}, \quad x, y \in(0, \infty)^{m}, x \neq y .
\end{aligned}
$$

We have changed the power $3 / 4$ of $t$ by $1 / 2$ in Lemma 3.2 , (b), when it was needed (see its proof).

Also as in Lemma 3.1 . $(a),(b),(d)$; and Lemma 3.2 , (a); we get

$$
\int_{0}^{\infty}\left|S_{t}^{3}(x, y ; a)\right| \frac{d t}{\sqrt{t}} \leq \frac{C}{\sqrt{a}} \int_{0}^{\infty} \frac{e^{-c a|x-y|^{2} / t}}{t^{(m+2) / 2}} d t \leq \frac{C}{a^{(m+1) / 2}|x-y|^{m}}, \quad x, y \in(0, \infty)^{m}, x \neq y
$$

In Lemma 3.1. $(b)$, the factor $\left(\mathfrak{a}(t) a x_{k} y_{k}\right)^{-1}$ has been removed when it was less than 1 .

We obtain as in Lemma 3.1. $(a)$ and $(f)$, and Lemma 3.2. $(a)$,

$$
\int_{0}^{\infty}\left|S_{t}^{2}(x, y ; a)\right| \frac{d t}{\sqrt{t}} \leq \frac{C}{\sqrt{a}} \int_{0}^{\infty} \frac{e^{-c a|x-y|^{2} / t}}{t^{(m+2) / 2}} d t \leq \frac{C}{a^{(m+1) / 2}|x-y|^{m}}, \quad x, y \in(0, \infty)^{m}, x \neq y
$$

On the other hand, from Lemma 3.1. $(a)$; and Lemma 3.2. $(d),(e)$ and $(f)$; we deduce that

$$
\int_{0}^{\infty}\left|T_{t}^{1}(x, y ; a)\right| \frac{d t}{\sqrt{t}} \leq \frac{C}{a^{(m+1) / 2}|x-y|^{m}}, \quad x, y \in(0, \infty)^{m}, \quad x \neq y .
$$

Moreover, by Lemma 3.1, (a) and (b); and Lemma 3.2, (c),

$$
\int_{0}^{\infty}\left|T_{t}^{2}(x, y ; a)\right| \frac{d t}{\sqrt{t}} \leq \frac{C}{a^{(m+1) / 2}|x-y|^{m}}, \quad x, y \in(0, \infty)^{m}, \quad x \neq y
$$

Putting together (72), 73), (74), 75), (76) and (77), we conclude that $(71$ holds.

The following property holds

$$
\begin{aligned}
\sup _{a \in \mathbb{R} \backslash\{0\}} & \left\{\left|\nabla_{x}\left(a \frac{d}{d a}\left(R_{\alpha, 1}(x, y ; a)-R_{1}(x, y ; a)\right)\right)\right|+\left|\nabla_{y}\left(a \frac{d}{d a}\left(R_{\alpha, 1}(x, y ; a)-R_{1}(x, y ; a)\right)\right)\right|\right\} \\
\leq & \frac{C}{|x-y|^{m+1}}, \quad x, y \in(0, \infty)^{m}, x \neq y
\end{aligned}
$$

We now prove that

$$
\sup _{a \in \mathbb{R} \backslash\{0\}}\left|\partial_{x_{1}}\left(a \frac{d}{d a}\left(R_{\alpha, 1}(x, y ; a)-R_{1}(x, y ; a)\right)\right)\right| \leq \frac{C}{|x-y|^{m+1}}, \quad x, y \in(0, \infty)^{m}, \quad x \neq y
$$

The proof of 78 can be completed by the interested reader by using the same ideas used in the proof of 79 but making careful manipulations. 
Let $a>0$. According to 49 we have that, for every $x, y \in(0, \infty), x \neq y$

$$
\begin{aligned}
\partial_{x_{1}} & {\left[\partial_{a}\left(R_{\alpha, 1}(x, y ; a)-R_{1}(x, y ; a)\right)\right]=\frac{m}{2} a^{(m-2) / 2} \partial_{x_{1}}\left[R_{\alpha, 1}(\sqrt{a} x, \sqrt{a} y ; 1)-R_{1}(\sqrt{a} x, \sqrt{a} y ; 1)\right] } \\
& +\frac{a^{(m-1) / 2}}{2} \int_{0}^{\infty}\left\{\left[\left(\partial_{x_{1}} G_{t}^{\alpha_{1}}\right)\left(\sqrt{a} x_{1}, \sqrt{a} y_{1}\right)-\left(\partial_{x_{1}} G_{t}\right)\left(\sqrt{a} x_{1}, \sqrt{a} y_{1}\right)\right] \prod_{j=2}^{m} W_{t}^{\alpha_{j}}\left(\sqrt{a} x_{j}, \sqrt{a} y_{j}\right)\right. \\
& +x_{1} \sqrt{a}\left[\left(\partial_{x_{1}}^{2} G_{t}^{\alpha_{1}}\right)\left(\sqrt{a} x_{1}, \sqrt{a} y_{1}\right)-\left(\partial_{x_{1}}^{2} G_{t}\right)\left(\sqrt{a} x_{1}, \sqrt{a} y_{1}\right)\right] \prod_{j=2}^{m} W_{t}^{\alpha_{j}}\left(\sqrt{a} x_{j}, \sqrt{a} y_{j}\right) \\
& +\sqrt{a}\left[\left(\partial_{x_{1}} G_{t}^{\alpha_{1}}\right)\left(\sqrt{a} x_{1}, \sqrt{a} y_{1}\right)-\left(\partial_{x_{1}} G_{t}\right)\left(\sqrt{a} x_{1}, \sqrt{a} y_{1}\right)\right] \\
& \times \sum_{j=2}^{m}\left(x_{j}\left(\partial_{x_{j}} W_{t}^{\alpha_{j}}\right)\left(\sqrt{a} x_{j}, \sqrt{a} y_{j}\right)+y_{j}\left(\partial_{y_{j}} W_{t}^{\alpha_{j}}\right)\left(\sqrt{a} x_{j}, \sqrt{a} y_{j}\right)\right) \prod_{i=2, i \neq j}^{m} W_{t}^{\alpha_{i}}\left(\sqrt{a} x_{i}, \sqrt{a} y_{i}\right) \\
& \left.+\sqrt{a} y_{1}\left[\left(\partial_{x_{1} y_{1}}^{2} G_{t}^{\alpha_{1}}\right)\left(\sqrt{a} x_{1}, \sqrt{a} y_{1}\right)-\left(\partial_{x_{1} y_{1}}^{2} G_{t}\right)\left(\sqrt{a} x_{1}, \sqrt{a} y_{1}\right)\right] \prod_{j=2}^{m} W_{t}\left(\sqrt{a} x_{j}, \sqrt{a} y_{j}\right)\right\} \frac{d t}{\sqrt{t}} .
\end{aligned}
$$

According to (44) and [30] we obtain for every $x, y \in(0, \infty)^{m}, x \neq y$

$$
a^{(m-2) / 2}\left|\partial_{x_{1}}\left(R_{\alpha, 1}(\sqrt{a} x, \sqrt{a} y ; 1)-R_{1}(\sqrt{a} x, \sqrt{a} y ; 1)\right)\right| \leq \frac{C}{a|x-y|^{m+1}} .
$$

Then, Lemma 3.1. (a), and Lemma 3.2, (d), lead to

$$
\begin{gathered}
a^{(m-1) / 2} \int_{0}^{\infty}\left|\left(\partial_{x_{1}} G_{t}^{\alpha_{1}}\right)\left(\sqrt{a} x_{1}, \sqrt{a} y_{1}\right)-\left(\partial_{x_{1}} G_{t}\right)\left(\sqrt{a} x_{1}, \sqrt{a} y_{1}\right)\right| \prod_{j=2}^{m} W_{t}^{\alpha_{j}}\left(\sqrt{a} x_{j}, \sqrt{a} y_{j}\right) \frac{d t}{\sqrt{t}} \\
\leq \frac{C}{a|x-y|^{m+1}}, \quad x, y \in(0, \infty)^{m}, \quad x \neq y .
\end{gathered}
$$

Moreover, we apply Lema 3.2 . $(g)$, to obtain, for each $x, y \in(0, \infty)^{m}, x \neq y$,

$$
a^{m / 2} x_{1} \int_{0}^{\infty}\left|\partial_{x_{1}}^{2}\left(G_{t}-G_{t}^{\alpha_{1}}\right)\left(\sqrt{a} x_{1}, \sqrt{a} y_{1}\right)\right| \prod_{j=2}^{m} W_{t}^{\alpha_{j}}\left(\sqrt{a} x_{j}, \sqrt{a} y_{j}\right) \frac{d t}{\sqrt{t}} \leq \frac{C}{a|x-y|^{m+1}}
$$

By using Lemma 3.1. $(a)$ and $(e)$; and by proceeding as in the previous cases we obtain

$$
\begin{gathered}
a^{m / 2} \int_{0}^{\infty}\left|\left(\partial_{x_{1}} G_{t}^{\alpha_{1}}\right)\left(\sqrt{a} x_{1}, \sqrt{a} y_{1}\right)-\left(\partial_{x_{1}} G_{t}\right)\left(\sqrt{a} x_{1}, \sqrt{a} y_{1}\right)\right| \sum_{j=2}^{m} \prod_{i=2, i \neq j}^{m} W_{t}\left(\sqrt{a} x_{i}, \sqrt{a} y_{i}\right) \\
\quad \times\left|x_{j}\left(\partial_{x_{j}} W_{t}^{\alpha_{j}}\right)\left(\sqrt{a} x_{j}, \sqrt{a} y_{j}\right)+y_{j}\left(\partial_{y_{j}} W_{t}^{\alpha_{j}}\right)\left(\sqrt{a} x_{j}, \sqrt{a} y_{j}\right)\right| \frac{d t}{\sqrt{t}} \\
\leq C a^{(m-1) / 2} \int_{0}^{\infty} \frac{e^{-c a|x-y|^{2} / t}}{t^{(m+3) / 2}} d t \leq \frac{C}{a|x-y|^{m+1}}, \quad x, y \in(0, \infty)^{m}, \quad x \neq y .
\end{gathered}
$$

Finally, as in the proof of 82 we get

$$
\begin{gathered}
a^{m / 2} \int_{0}^{\infty} y_{1}\left|\left(\partial_{y_{1} x_{1}}^{2} G_{t}^{\alpha_{1}}\right)\left(\sqrt{a} x_{1}, \sqrt{a} y_{1}\right)-\left(\partial_{y_{1} x_{1}}^{2} G_{t}\right)\left(\sqrt{a} x_{1}, \sqrt{a} y_{1}\right)\right| \prod_{j=2}^{m} W_{t}\left(\sqrt{a} x_{j}, \sqrt{a} y_{j}\right) \frac{d t}{\sqrt{t}} \\
\leq \frac{C}{a\left(|x-y|^{m+1}\right)}, \quad x, y \in(0, \infty)^{m}, x \neq y .
\end{gathered}
$$

Putting together 80, 81, 82, 83, and 84, we deduce

$$
\left|\partial_{x_{1}} \partial_{a}\left(R_{\alpha, 1}(x, y ; a)-R_{1}(x, y ; a)\right)\right| \leq \frac{C}{a|x-y|^{m+1}}, \quad x, y \in(0, \infty)^{m}, x \neq y .
$$

If $a<0$ we can proceed in a similar way and 79 is established.

According to [16, Proof of Lemma 2.2 and Proposition 2.3], we have that

$$
\sup _{a \in \mathbb{R} \backslash\{0\}}\left|a \frac{d}{d a} R_{1}(x, y ; a)\right| \leq \frac{C}{|x-y|^{m}}, \quad x, y \in(0, \infty)^{m}, x \neq y,
$$


and

$$
\sup _{a \in \mathbb{R} \backslash\{0\}}\left\{\left|\nabla_{x}\left(a \frac{d}{d a} R_{1}(x, y ; a)\right)\right|+\left|\nabla_{y}\left(a \frac{d}{d a} R_{1}(x, y ; a)\right)\right|\right\} \leq \frac{C}{|x-y|^{m+1}}, \quad x, y \in(0, \infty)^{m}, x \neq y .
$$

Then, from (71) and 79 , we conclude that

$$
\sup _{a \in \mathbb{R} \backslash\{0\}}\left|a \frac{d}{d a} R_{\alpha, 1}(x, y ; a)\right| \leq \frac{C}{|x-y|^{m}}, \quad x, y \in(0, \infty)^{m}, x \neq y,
$$

and

$$
\sup _{a \in \mathbb{R} \backslash\{0\}}\left\{\left|\nabla_{x}\left(a \frac{d}{d a} R_{\alpha, 1}(x, y ; a)\right)\right|+\left|\nabla_{y}\left(a \frac{d}{d a} R_{\alpha, 1}(x, y ; a)\right)\right|\right\} \leq \frac{C}{|x-y|^{m+1}}, \quad x, y \in(0, \infty)^{m}, x \neq y \text {. }
$$

By [33, Theorem 1.3, Chapter XII] the proof of this lemma is finished.

\section{REFERENCES}

[1] J. Bergh and J. Löfström, Interpolation spaces. An introduction, Springer-Verlag, Berlin, 1976.

[2] J. J. Betancor, J. C. Fariña, L. Rodríguez-Mesa, and A. Sanabria, Higher order Riesz transforms for Laguerre expansions, Illinois J. of Math., 55 (2011), pp. 27-68.

[3] B. Bongioanni and J. L. Torrea, Sobolev spaces associated to the harmonic oscillator, Proc. Indian Acad. Sci. Math. Sci., 116 (2006), pp. 337-360.

[4] —. What is a Sobolev space for the Laguerre function systems?, Studia Math., 192 (2009), pp. 147-172.

[5] P. Chen And A. Sikora, Sharp spectral multipliers for a new class of Grushin type operators. Preprint 2012 (arXiv: 1210.0322 v1)

[6] M. Christ, $L^{p}$ bounds for spectral multipliers on nilpotent groups, Trans. Amer. Math. Soc., 328 (1991), pp. 7381.

[7] R. R. Coifman and G. Weiss, Analyse harmonique non-commutative sur certains espaces homogènes, Lecture Notes in Mathematics, Vol. 242, Springer-Verlag, Berlin, 1971.

[8] X. T. Duong, E. M. Ouhabaz, and A. Sikora, Plancherel-type estimates and sharp spectral multipliers, J. Funct. Anal., 196 (2002), pp. 443-485.

[9] X. T. Duong, A. Sikora, And L. Yan, Weighted norm inequalities, Gaussian bounds and sharp spectral multipliers, J. Funct. Anal., 260 (2011), pp. 1106-1131.

[10] K. Engel and R. Nagel, One-parameter semigroups for linear evolution equations, vol. 194 of Graduate Texts in Mathematics, Springer-Verlag, New York, 2000.

[11] E. B. Fabes, C. E. Gutiérrez, and R. Scotto, Weak-type estimates for the Riesz transforms associated with the Gaussian measure, Rev. Mat. Iberoamericana, 10 (1994), pp. 229-281.

[12] G. B. Folland and E. M. Stein, Hardy spaces on homogeneous groups, vol. 28 of Mathematical Notes, Princeton University Press, Princeton, N.J., 1982.

[13] E. Harboure, J. L. Torrea, and B. E. Viviani, Riesz transforms for Laguerre expansions, Indiana Univ. Math. J., 55 (2006), pp. 999-1014.

[14] G. H. Hardy, J. E. Littlewood, and G. Pólya, Inequalities, Cambridge, at the University Press, 1934.

[15] W. Hebisch, A multiplier theorem for Schrödinger operators, Colloq. Math., 60/61 (1990), pp. 659-664.

[16] K. Jotsaroop, P. Sanjay, and S. Thangavelu, Riesz transforms and multipliers for the Grushin operator. To appear in J. Analyse Math. (arXiv:1110.3227 J1)

[17] N. N. Lebedev, Special functions and their applications, Dover Publications Inc., New York, 1972.

[18] C. Martínez Carracedo and M. Sanz Alix, The theory of fractional powers of operators, vol. 187 of NorthHolland Mathematics Studies, North-Holland Publishing Co., Amsterdam, 2001.

[19] A. Martini and D. Müller, A sharp multiplier theorem for Grushin operators in arbitrary dimensions. Preprint 2012 (arXiv:1210.3564 1 ).

[20] A. Martini And A. Sikora, Weighted Plancherel estimates and sharp spectral multipliers for the Grushin operators. To appear in Math. Res. Lett. (arXiv:1204.1159v1)

[21] S. Meda, A general multiplier theorem, Proc. Amer. Math. Soc., 110 (1990), pp. 639-647.

[22] B. Muckenhoupt and E. M. Stein, Classical expansions and their relation to conjugate harmonic functions, Trans. Amer. Math. Soc., 118 (1965), pp. 17-92.

[23] B. Muckenhoupt And D. W. WebB, Two-weight norm inequalities for the Cesàro means of Hermite expansions, Trans. Amer. Math. Soc., 354 (2002), pp. 4525-4537 (electronic).

[24] A. Nowak, Heat-diffusion and Poisson integrals for Laguerre and special Hermite expansions on weighted $L^{p}$ spaces, Studia Math., 158 (2003), pp. 239-268.

[25] A. Nowak and K. Stempak, Riesz transforms and conjugacy for Laguerre function expansions of Hermite type, J. Funct. Anal., 244 (2007), pp. 399-443.

[26] — On L L $^{p}$-contractivity of Laguerre semigroups. To appear in Illinois J. of Math. (arXiv:1011.5437 J1)

[27] E. M. Ouhabaz, Gaussian estimates and holomorphy of semigroups, Proc. Amer. Math. Soc., 123 (1995), pp. $1465-1474$.

[28] A. Sikora, Multivariable spectral multipliers and analysis of quasielliptic operators on fractals, Indiana Univ. Math. J., 58 (2009), pp. 317-334.

[29] A. Sikora and J. Wright, Imaginary powers of Laplace operators, Proc. Amer. Math. Soc., 129 (2001), pp. 1745-1754 (electronic).

[30] K. Stempak and J. L. Torrea, Poisson integrals and Riesz transforms for Hermite function expansions with weights, J. Funct. Anal., 202 (2003), pp. 443-472. 
[31] G. Szegö, Orthogonal polynomials, American Mathematical Society, Providence, R.I., fourth ed., 1975. American Mathematical Society, Colloquium Publications, Vol. XXIII.

[32] S. Thangavelu, Lectures on Hermite and Laguerre expansions, vol. 42 of Mathematical Notes, Princeton University Press, Princeton, NJ, 1993.

[33] A. Torchinsky, Real-variable methods in harmonic analysis, vol. 123 of Pure and Applied Mathematics, Academic Press Inc., Orlando, FL, 1986.

[34] L. Weis, Operator-valued Fourier multiplier theorems and maximal $L_{p}$-regularity, Math. Ann., 319 (2001), pp. $735-758$.

[35] A. YAGI, Coïncidence entre des espaces d'interpolation et des domaines de puissances fractionnaires d'opérateurs, C. R. Acad. Sci. Paris Sér. I Math., 299 (1984), pp. 173-176.

Víctor Almeida, Jorge J. Betancor, Alejandro J. Castro

Departamento de Análisis Matemático, Universidad de la laguna,

Campus de Anchieta, Avda. Astrofísico Francisco Sánchez, s/n,

38271, La Laguna (Sta. Cruz de Tenerife), Spain

E-mail address: valmeida@ull.es, jbetanco@ull.es, ajcastro@ull.es

Kishin Sadarangani

Departamento de Matemáticas, Universidad de Las Palmas de Gran Canaria,

Campus de Tafira Baja,

35017, Las Palmas de Gran Canaria, Spain

E-mail address: ksadaran@dma.ulpgc.es 